I. Nakamura

Nagoya Math. J.

Vol. 58 (1975), 149-214

\title{
ON MODULI OF STABLE QUASI ABELIAN VARIETIES
}

\author{
IKU NAKAMURA
}

\section{Introduction}

In [4] Deligne-Mumford introduced a concept of stable curves and proved the existence of the coarse moduli space of stable curves of given genus in the category of algebraic stacks. Thereafter Mumford has shown this coarse moduli space is a projective scheme. We can consider the coarse moduli space of stable curves as a geometric compactification of the coarse moduli space of non-singular curves.

On the other hand Mumford showed the existence of the fine moduli scheme $(\lambda \geqq 3)$, the coarse moduli scheme $(\lambda=1,2)$ of polarized abelian schemes with degree $d$ and level $\lambda$-structures as a consequence of his general theory on the existence of the quotient. ([12]) Also Igusa obtained the same result in the case of dimension one before Mumford. ([10]) Recently Deligne-Rapport has proved that the fine moduli scheme of generalized elliptic curves with level $\lambda$ structures exists if $\lambda \geqq 3$ and becomes a projective scheme. ([5]) A generalized elliptic curve with level $\lambda$ structure is one of the following, a nonsingular elliptic curve with level $\lambda$ structure, a chain of $\lambda$ lines with level $\lambda$ structure. In this case the moduli of generalized elliptic curves with level $\lambda$ structures is considered as a geometric compatification of the moduli of elliptic curves with level $\lambda$ structures.

The main purpose of the present paper is to construct a geometric compactification of the moduli space of principally polarized abelian schemes with level $\lambda$-structures over the complex number field in the lower dimensional cases. Namely we define a principally polarized stable quasi-abelian scheme (or variety) with level $\lambda$ structure (abbr. SQAS or $\mathrm{SQAV})(\S 2)$ and construct a compactification of the moduli of principally polarized abelian schemes by adding the isomorphism classes of SQAS. A principally polarized non singular stable quasi abelian scheme

\footnotetext{
Received February 8, 1975.
} 
with level $\lambda$-structure is by definition a principally polarized abelian scheme of the same level.

Our main theorem can be stated as follows,

Theorem 6.2. $(g=2,3)$

(i) The functor $\widetilde{S}_{g}(\lambda)$, (more precisely the sheafification of the functor) of principally polarized stable quasi abelian schemes with level $\lambda$ structures over $C$ of dimension $g$ is represented by a non singular nonseparated algebraic space $S_{g}^{*}(\lambda)$ locally of finite presentation if $\lambda \geqq 3$. The greatest separated quotient $S_{g}(\lambda)$ of $S_{g}^{*}(\lambda)$ exists and is isomorphic with the Igusa monoidal transform $\mathfrak{S}_{g}^{*}(\lambda)$ of Satake compactification of the Siegel modular space $\mathfrak{S}_{g}(\lambda)$ of level $\lambda$.

(ii) $\widetilde{\Im}_{g}^{*}(\lambda)(\lambda=1,2)$ has the following property,

(1) Given a SQAS over a scheme $S$ of finite type over $C$ there exists a unique morphism from $S$ to $\widetilde{\Im}_{g}^{*}(\lambda)$ in the category of algebraic spaces.

(2) For any separated algebraic space $N$ enjoying the property (1), a unique morphism $g$ from $\widetilde{\Xi}_{g}^{*}(\lambda)$ to $N$ is defined in such a way that the following diagram commutes for any SQAS over $S$,

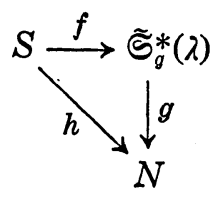

where $h$ is morphism defined by the property (1).

We remark that $\widetilde{\Im}_{g}^{*}(\lambda)$ is known to be compact. A remarkable fact is that the moduli space $S_{g}^{*}(\lambda)$ is not separated, contrary to the cases of stable curves, generalized elliptic curves. It comes mainly from the fact that a SQAS of level 1 is not necessarily irreducible and an ample invertible sheaf on a SQAS does not seem to be determined canonically. A concept of a stable quasi abelian variety was first obtained by Ueno in case of $g=2$ in his unpublished manuscript [30]. He constructed the degenerate fibers of principally polarized abelian varieties by using theta functions. After Ueno the author obtained stable quasi-abelian varieties as a consequence of properfication of analytic Neron model in case of $g=2,3$. After their works Namikawa gave two definitions of SQAS, one of which the author also obtained around the same time time independently. Two definitions of SQAS are proved to coincide with each 
other if a certain arithmetic condition (A) is satisfied. (proposition 2.2.) If $g$ is not greater than four, (A) holds always true. (lemma 1.5.) If $g$ is greater than four, this may not be the case. Therefore we shall give a precise definition of SQAS only under the condition (A). If this condition (A) fails, then our SQAS is not reduced. (proposition 2.4.) It seems to the author that there is no reason to determine which definition of SQAS is better when the condition (A) fails. So we have only candidates for a genuine SQAS in the general case. Our definition of SQAS is greatly indebted to Mumford [13]. (definition 2.3.) On the other hand in the analytic case, the fine (or coarse) moduli of principally polarized abelian varieties with level $\lambda$ structures is known to be the Siegel space $\mathfrak{S}_{g}^{*}(\lambda)$ of level $\lambda$. The compactification of it was considered by many people. The most famous compactification is due to Satake, which is often called the Satake compactification. Igusa investigated the structure of the blowing up $\widetilde{\Im}_{g}^{\infty}(\lambda)$ of the Satake compactification by the ideal of cusp forms and showed that the blowing up is non-singular when $g \leqq 3$, singular when $g>3$. In this article we call the last compactification $\widetilde{S}_{g}^{*}(\lambda)$ of $\widetilde{S}_{g}(\lambda)$ the Igusa monoidal transform of the Satake compactification in accordance with [16]. Recently a new method of compactifying symmetric domains has been found by Mumford [14] and Satake [28]. Mumford has shown a general idea of compactifying the Siegel space in the former half of [14]. In [18], as he himself stated at the end, Namikawa gave a false proof of a theorem that a canonical morphism from the coarse moduli space of nonsingular curves to the Siegel space can be extended to a morphism from the coarse moduli space of stable curves to the Igusa monoidal transform. Mumford told Namikawa in his letter that Namikawa's theorem and proof can be modified into correct ones by taking a partial compactification of the Siegel space associated with $D V$ cones instead of the Igusa monoidal transform. We notice that the original theorem in [18] has not been known to be true or not yet. Stimulated with this Namikawa gave a precise construction of a new compactification of the Siegel space associated with $D V$ cones which was named the Voronoi compatification. The Voronoi compactification is the same as the Igusa monoidal transform for $g \leqq 3$. The Voronoi compatification is deeply related with our stable quasiabelian varieties. In fact, our main theorem states that if $g \leqq 3$, the Voronoi compactification is in a wide sense the moduli space of SQAS. 
Moreover Namikawa has succeeded in constructing a polarized family of SQAV's of level $\underline{\lambda}$ over the Voronoi compactification of level $2 \lambda$. From these facts it is conjectured that the functor $\mathscr{S}_{g}(\lambda)$ (possibly with a slight modification) is represented by an algebraic space $S_{g}^{*}(\lambda)$ of finite type and its greatest separated quotient $S_{g}(\lambda)$ exists and is isomorphic with the Voronoi compactification even for $g$ greater than three. Now we shall explain an outline of this article.

In $\S 1$, we prepare definitions, some lemmas and propositions. The results in this section are quite elementary. In $\S 2$ we shall define SQAS $X$ under an arithmetic condition (A) and prove the fundamental properties of SQAS. For instance, $X$ is reduced and Cohen-Macaulay under the condition (A). Moreover we shall see that the configuration of $X$ is given by a certain kind of a polyhedral decomposition of $g$ dimensional Euclidean space, which is called Delone decomposition. Most of the properties of $X$ can be described by this Delone decomposition associated with $X$. Furthermore we shall give two important exact sequences of coherent sheaves on $X$, and calculate the automorphism group of $X$ under the conditions (A) and (B). Then the connected component of the automorphism group of $X$ has dimension $g$ (theorem 2.1.). We remark that the conditions (A) and (B) do hold if $g \leqq 4$. (lemma 1.5.)

In $\S 3$ we shall give an outline of the construction of the Voronoi compactification and Namikawa's polarized family of SQAV over the Voronoi compactification. In the rest of this section we shall make a short investigation of the boundary of the Voronoi compactification in the lower dimensional cases from the view point of troidal embeddings.

In $\S 4, \S 5$, we shall calculate $\operatorname{Ext}^{1}\left(\Omega_{X}^{1} \mathcal{O}_{X}\right)$ for a SQAS of level 1 ( $g=$ $2,3)$ which is the tangent space of the deformation functor of $X$ because $X$ is a reduced proper scheme. ([24]) For that purpose we use a spectral sequence $E_{2}^{p q}=H^{p}\left(X \mathscr{E}_{x x} t^{q}\left(\Omega_{X}^{1} \mathcal{O}_{X}\right)\right)$ converging to $\mathscr{E}_{x t} t^{p+q}\left(\Omega_{X}^{1} \mathcal{O}_{X}\right)$. Using the results in $\S 2$, we can compute the terms $E_{2}^{1,0}$ and $E_{2}^{0,1}$. For a SQAS of dimension 3 whose corresponding Delone decomposition is not the composite of Delone decompositions of lower dimension we have an interesting formula, $\operatorname{dim} E_{2}^{1,0}=3+N_{0}-N_{1}+2 N_{2}-3 N_{3}$ where $N_{\nu}$ denotes the number of $\nu$ dimensional Delone cells modulo translations by integral vectors. ((5.6.) Remark)

In order to compute the term $E_{2}^{0,1}$ we introduce a concept of the graph of $X$, which should be called the graph of the singular loci of $X$. 
If $g$ is not greater than three we have $\operatorname{dim} E_{2}^{0,1}=$ the number of connected components of the graph of $X$. (Corollary of Lemma 5.3.)

From these calculations, the spectral sequence mentioned above implies an inequality $\operatorname{dim} \operatorname{Ext}^{1}\left(\Omega_{X}^{1} \mathcal{O}_{X}\right) \leqq g^{2}$ if $g=2,3$. On the other hand we can construct a flat family of deformations of $X$ depending on $g^{2}$ effective parameters so that we have $\operatorname{dim} \operatorname{Ext}^{1}\left(\Omega_{X}^{1} \mathcal{O}_{X}\right)=g^{2}(g=2,3)$.

In $\S 6$, we shall define a functor $\mathscr{S}_{g}(\lambda)$, i.e., the sheafification of the functor of SQAS of level $\lambda$, and show our main theorem. For this purpose we shall consider a functor $D_{L}$, in short, the deformation functor with polarization. (lemma 6.4.) With the aid of lemma 6.4. and Wavrik's theorem, we shall prove that $\mathscr{S}_{g}(\lambda)$ is locally effectively prorepresentable by complete regular local rings of dimension $\frac{1}{2} g(g+1)$. From these results we infer our main theorem along the same line of arguments as that of Deligne-Rapoport [5]. The representability is shown by using a generalization of a theorem of Artin by DeligneRapoport [5].

In $\S 7$ we shall give a brief account of the reason why we have taken our definition of SQAS instead of degenerate fibers appearing in Deligne's example [13]. Moreover we shall show an example of a construction of a local family of stable curves. On the last topic we shall discuss in full details in the subsequent paper [16]. In fact the subsequent paper [16] was the starting point of the present paper.

The author would like to express his hearty thanks to Dr. Namikawa, Dr. Umemura, Dr. Ueno and Dr. Kashiwara and other mathematicians in Nagoya University for their valuable advices and encouragements. Dr. Namikawa has kindly shown the author his handwritten manuscript.

\section{Notations.}

Let $E$ be a real vector space of dimension $N$ with a fixed isomorphism $E \cong R^{N}$. Define $E_{Z}=Z^{N}$ through this isomorphism. $E$ is equipped with the inner product $\left\langle x_{1}, x_{2}\right\rangle=x_{1}^{t} x_{2}\left(x_{i} \in E\right)$. Let $C$ be a cone in $E$. $\check{C}:=\left\{x \in E ;\left\langle x, x^{\prime}\right\rangle \geqq 0\right.$ for any $\left.x^{\prime} \in C\right\}$

$(\check{C})_{z}:=\check{C} \cap E_{z}$

$\boldsymbol{R}^{+} D:=\left\{r x ; r \in \boldsymbol{R}^{+}, x \in D\right\} \quad(D \subset E)$

$\boldsymbol{Q}^{+} D:=\left\{r x ; r \in \boldsymbol{Q}^{+}, x \in D\right\}$

$\mathfrak{Y}_{g}^{+}$: the cone of real symmetric positive definite matrices of degree $g$.

$\Sigma: \quad D-V$ cone (1.1.) 
$\sigma:$ Delone cell (1.1.)

$V_{\Sigma \sigma}$ : a mixed $D-V$ cone associated with $\Sigma$ and $\sigma$ (3.2.)

$\left(V_{\Sigma_{\sigma}}\right)_{Z}:=\left\{(y, x) \in \overline{\mathfrak{Y}}_{g}^{+} \times \boldsymbol{R}^{g} ; y:\right.$ half integer, $x$ : integral $\} \cap V_{\Sigma_{\sigma}}$ where $y$ is called a half integer matrix if $y_{i i} \in Z, 2 y_{i j} \in Z(i \neq j)$.

${ }^{t} u$ : the transposed matrix of $u$.

$Z_{0}^{+}$: non negative integers

$\Phi_{g}$ : the set of Delone $g$ cells containg the origin

$\Phi$ : the set of Delone 1 cells containg the origin

$\tau \prec \sigma: \quad \tau$ is a face of $\sigma$

$[\sigma: \tau]$ : the incidence number, where $\tau$ is a one codimensional face of $\sigma$ $M_{g}(Z)$ : the ring of integral matrices of degree $g$.

$G L(g Z)$ : the group of integral matrices of degree $g$ with determinant $\pm 1$

$\Gamma_{g}$ or $S p(g Z):=\left\{M \in M_{2 g}(Z) ; M J^{t} M=J\right\}$ where $J=\left(\begin{array}{ll}0 & 1_{g} \\ -1_{g} & 0\end{array}\right), \Gamma_{g}(\lambda)$ or $\operatorname{Sp}(g Z)(\lambda):=\left\{M \in \operatorname{Sp}(g Z) ; M \equiv 1_{2 g} \bmod \lambda\right\}$

$\varsigma_{g}$ : the Siegel upper half plane of degree $g$ (2.1.)

$\mathfrak{S}_{g}^{*}(\lambda):=\widetilde{\varsigma}_{g} / \Gamma_{g}(\lambda)$ the Siegel space of level $\lambda$

$\widetilde{\Im}_{\theta}^{*}(\lambda)$ : the Voronoi compactification of level $\lambda$

$e(x):=\exp (2 \pi \sqrt{-1} x)$

$\zeta:=\left(\zeta_{1}, \cdots, \zeta_{g}\right)$ variable

$m:=\left(m_{1}, \cdots, m_{g}\right) \in \boldsymbol{Z}^{g}$

$R(\tau):=\mathcal{O}\left[\xi_{m} \cdot \theta m \in Z^{g}\right] \xi_{m}=e\left(\frac{1}{2} m \tau\left(s^{2}\right)^{t} m+m^{t} \zeta\right)$

$\tilde{R}(\tau):=\mathcal{O}\left[\tilde{\xi}_{m} \cdot \theta m \in Z^{g}\right] \tilde{\xi}_{m}=e\left(\frac{1}{2} m \tau(s)^{t} m+m^{t}\left(\zeta+\frac{1}{2} r(s)\right)\right)$ where $r(s)=$ $\left(r_{i}(s)\right), r_{i}(s)=\tau_{i i}(s) \quad$ (2.4. Remark)

$R^{*}(\tau):$ (2.4.)

$\left(\mathfrak{Y}_{g}^{+}\right)_{\boldsymbol{Z}}:=\left\{y \in \mathfrak{Y}_{g}^{+} ; y:\right.$ half integer $\}$

$\overline{\mathfrak{Y}}_{g}^{+}$: the convex hull of all symmetric positive semi-definite integral matrices of degree $g$ over $\boldsymbol{R}^{+}$

Table of Contents

$\S 1$. Delone cell, Voronoi cell, D-V cone and a semigroup $S_{0}$

§. SQAV

$\S 3$. The Voronoi compactification and Namikawa's polarized family of SQAV over it

$\S 4$. Infinitesimal deformations of SQAV (1)

$\S 5$. Infinitesimal deformations of SQAV (2)

$\S 6$. Moduli of SQAV $(g \leqq 3)$ 


\section{§7. Further remarks}

\section{$\S 1$ Delone cell, Voronoi cell, $D-V$ cone and a semigroup $S_{0}$.}

(1.1) For a matrix $y$ of $\overline{\mathfrak{Y}}_{g}^{+}$, we define two decompositions of $\boldsymbol{R}^{g}$ as follows,

Definition 1.1. ([18]) A subset $\sigma$ of $\boldsymbol{R}^{g}$ is called a Delone cell w.r.t. $y$ if there exist integral vectors $a_{j}\left(\in Z^{g}\right)$ and $\alpha \in \boldsymbol{R}^{g}$ such that

(0) $\sigma=\left\{\sum_{i=1}^{r} \lambda_{i} a_{j_{i}} ; 1 \leqq \lambda_{i} \leqq 0, \sum_{i=1}^{r} \lambda_{i}=1, j_{i} \in I, r=1,2, \cdots\right\}$

(1) $(a-\alpha) y^{t}(a-\alpha) \geqq c$ for some constant $c$

(2) in (1), equality holds if and only if $a=a_{j}$ for some $j$.

Definition 1.2. ([18]) A subset $\Delta=\Delta(\sigma)$ of $\boldsymbol{R}^{g}$ is called a Voroni cell (corresponding to $\sigma$ ) w.r.t. $y$ if

$$
\Delta(\sigma)=\{-2 \alpha y ;(0),(1),(2) \text { hold for } \alpha \text { and } \sigma\} .
$$

DEFINITION 1.3. All Delone cells (resp. Voronoi cells) w.r.t. $y$ give a polyhedral decomposition of $\boldsymbol{R}^{g}$ (resp. $\boldsymbol{R}^{g} y$ ), which is called Delone decomposition (resp. Voronoi decomposition) w.r.t. $y$.

We recall the fundamental properties of Delone or Voronoi decomposition.

Proposition 1.1. ([18]) The Delone (or Voronoi) decomposition has the following properties.

(1) Each cell has a finite number of faces, each of which is also a cell.

(2) The translation by an integral vector (resp. an integral vector times -2y) transforms a Delone cell (resp. a Voronoi cell) to another. The number of cells modulo translations is finite.

(3) If $y>0, \sigma$ is bounded and Delone cells of $\operatorname{dim} 0$ are $Z^{g} . \Delta$ is always bounded.

(4) If $y=\left(\begin{array}{cc}0 & 0 \\ 0 & y^{\prime \prime}\end{array}\right), y^{\prime \prime}>0$ rank $y^{\prime \prime}=g^{\prime \prime}$, then $\sigma=\boldsymbol{R}^{g^{\prime}} \times \sigma^{\prime \prime}, \Delta=0 \times \Delta^{\prime \prime}$ where $\sigma^{\prime \prime}$ (resp. $\left.\Delta^{\prime \prime}\right)$ is a Delone (resp. Voronoi) cell w.r.t. $y^{\prime \prime}, g=g^{\prime}+g^{\prime \prime}$. And $\sigma$ corresponds to $\Delta$ if and only if $\sigma^{\prime \prime}$ does to $\Delta^{\prime \prime}$.

(5) $\operatorname{dim} \sigma+\operatorname{dim} \Delta(\sigma)=g$

(6) $\sigma$ (resp. A) is a Delone (resp. Voronoi) cell w.r.t. $y$ if and only if $\sigma u^{-1}$ (resp. $\left.\Delta^{t} u\right)$ is so w.r.t. $u y^{t} u$. $(u \in G L(g Z))$ 
Proof. See Namikawa [18].

Now we introduce two conditions (A) and (B) about the Delone decomposition w.r.t. $y>0$ as follows,

For a Delone cell $\sigma$ containing the origin, we define $\boldsymbol{R}^{+} \sigma=\{\lambda a ; a \in \sigma$, $\lambda>0\}$.

(A) For any Delone cell $\sigma$, the vertices of $\sigma$ distinct from the origin generate $\boldsymbol{R}^{+} \boldsymbol{\sigma} \cap \boldsymbol{Z}^{g}$ as a semigroup.

(B) Let $\sigma_{1}$ and $\sigma_{2}$ be any pair of adjacent Delone cells of dimension $g$ i.e. $\sigma_{1} \cap \sigma_{2}=\tau(\tau$ : a Delone $(g-1)$ cell).

Then there exists a translate $\sigma_{1}^{\prime}$ of $\sigma_{1}$ by an integral vector such that $\sigma_{1}^{\prime} \cap \sigma_{2}$ contains at least a Delone 1 cell not lying on $\tau$.

Remark. As we see later, two conditions (A) and (B) hold at least if $g \leqq 4$.

Definition 1.4. A subset $\Sigma$ of $\overline{\mathfrak{Y}}_{g}^{+}$is called a Delone-Voronoi cone (abbr. D-V cone) if there exists $y_{0} \in \overline{\mathfrak{Y}}_{g}^{+}$

$$
\Sigma=\left\{y \in \overline{\mathfrak{Y}}_{g}^{+} ; y \text { is equivalent to } y_{0}\right\}
$$

where "equivalent" means that $y$ determines the same Delone decomposition as $y_{0}$ does.

THEOREM 1.1 (Voronoi [26]). All D-V cones form a cone decomposition of $\overline{\mathfrak{Y}}_{g}^{+}$which is admissible, namely, satisfies the following conditions,

(0) $\overline{\mathfrak{Y}}_{g}^{+}=\cup \Sigma_{i} \quad$ (disjoint union)

(1) $\Sigma_{i}$ is a rational convex cone, i.e., a convex cone generated by a finite number of integral matrices,

(2) every face of $\Sigma_{i}$ is also a $D-V$ cone

(3) this decomposition is invariant under the action of $G L(g Z)$

(4) the number of cosets w.r.t. $G L(g Z)$ if finite.

EXAMPLE. In case of $g=2$, all V-D cones modulo $G L(2 Z)$ are listed as follows
(0) $\{0\}$,
(1) $\left\{\left(\begin{array}{ll}0 & 0 \\ 0 & 0\end{array}\right) ; y>0\right\}$,
(2) $\left\{\left(\begin{array}{cc}y_{1} & 0 \\ 0 & y_{2}\end{array}\right), y_{1}, y_{2}>0\right\}$
(3) $\left\{\left(\begin{array}{cc}y_{1}+y_{3} & -y_{3} \\ y_{1}-y_{3} & y_{2}+y_{3}\end{array}\right) ; \begin{array}{l}y_{i}>0 \\ i=1,2,3\end{array}\right\}$. 
We list Delone decompositions determined by D-V cones shown above.

(1)

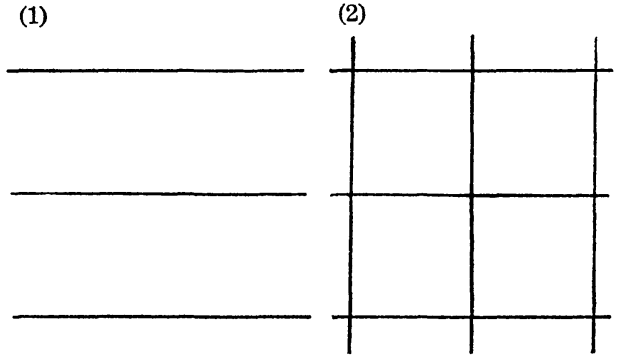

(3)

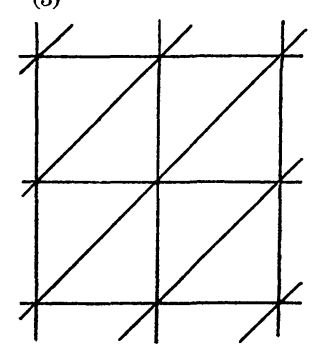

Lemma 1.1. Assume $\Sigma, \Sigma^{\prime}$ to be $D-V$ cones in $\mathfrak{Y}_{g}^{+}$. Then the Delone decomposition defined by $\Sigma$ is a polyhedral subdivision of that defined by $\Sigma^{\prime}$ if and only if $\Sigma^{\prime}$ is a face of $\Sigma$.

Proof. Let $\Phi_{g}$ be the set of all Delone $g$ cells w.r.t. $\Sigma$ containing the origin. Take a $g$ cell $\sigma$ of $\Phi_{g}$, and let the vertices of $\sigma$ be $a_{0}=0$, $a_{1}, \cdots, a_{r}$. Let $y$ be an element of $\Sigma$. Then there exists by definition a vector $\alpha\left(\in \boldsymbol{R}^{g}\right)$ such that

(1) $\left(a_{i}-\alpha\right) y^{t}\left(a_{i}-\alpha\right)=\left(a_{0}-\alpha\right) y^{t}\left(a_{0}-\alpha\right) \quad i=1, \cdots, r$.

(2) for an integral vector $a \neq a_{i}$,

$$
(a-\alpha) y^{t}(a-\alpha)>\left(a_{0}-\alpha\right) y^{t}\left(a_{0}-\alpha\right) .
$$

Hence we have,

(1) $a_{i} y^{t} a_{i}=2 \alpha y^{t} a_{i}$

(2) $\quad a y^{t} a>2 \alpha y^{t} a \quad\left(a \neq a_{i}\right)$

Since $\operatorname{dim} \sigma=g$ and $y>0, \alpha$ is uniquely determined by $\sigma$ and $y$. Put $\beta_{\Sigma}(y)=2 \alpha y . \quad \beta_{\Sigma}$ depends continuously on $y$ over the closure $\bar{\Sigma}$ of $\Sigma$ although $\alpha$ is not in general defined over $\bar{\Sigma}$. Any $y$ in $\Sigma$ satisfies (1)' and (2) for all $\sigma \in \Phi_{g}$. Conversely any $y$ in $\mathfrak{Y}_{g}^{+}$satisfying (1) and (2) for all $\sigma \in \Phi_{g}^{\prime}$ is contained in $\Sigma$ because the Delone decomposition w.r.t. $y$ is determined by $\Phi_{g}$. Assume that the Delone decomposition defined by another V-D cone $\Sigma$ is a polyhedral subdivision of that defined by $\Sigma^{\prime}$. Let $\Phi_{g}^{\prime}$ be the set of all Delone $g$-cells w.r.t. $\Sigma^{\prime}$ containing the origin. Then $\sigma \in \Phi_{g}^{\prime}$ is covered with a finite number of $g$-cells in $\Phi_{g}$. For instance assume $\sigma$ to be covered with $\sigma_{1}, \cdots, \sigma_{\ell}$, and the vertices of $\sigma_{i}$ to be $a_{0}$, 
$a_{1}^{(i)} \cdots a_{r_{i}}^{(i)}$. Hence $\left\{a_{0}, \cdots, a_{r}\right\}=\left\{a_{0}, a_{1}^{(i)}, \cdots, a_{r_{i}}^{(i)} i=1, \cdots, r\right\}$. Hence $\Sigma^{\prime}$ is obviously a face of $\Sigma$. Hence only if part is proved.

Next we shall prove if part. If $\Sigma^{\prime}$ is a face of $\Sigma$, then any $y$ in $\Sigma^{\prime}$ satisfies $(1)^{\prime}$, and some of $(2)^{\prime}$ may reduce to equalities. Since $\beta_{\Sigma}$ is defined over $\bar{\Sigma}, \beta_{\Sigma^{\prime}}(y)=\beta_{\Sigma}(y)$ for $y \in \Sigma^{\prime}$.

Hence the set of all $\alpha_{j}$ such that $a_{j} y^{t} a_{j}=\beta_{\Sigma}(y)^{t} \alpha_{j}$, contains $\alpha_{j}$ ( $j=$ $0, \cdots, r)$ as a part, i.e. the Delone decomposition w.r.t. $\Sigma$ is a polyhedral subdivision of that w.r.t. $\Sigma^{\prime}$.

Q.E.D.

LEMMA 1.2. Assume $U$ to be an open cone in $R^{N}$ defined by a fihite number of integral inequalities. Moreover suppose that there's no line completely contained in $U$. Set Aut $U=\{g \in G L(N Z) ; g \cdot U=U\}$. Then Aut $U$ is a finite group.

Proof. By assumption $U$ is the set defined by $a_{i}{ }^{t} x>0(i=1, \cdots, n)$ with integral vectors $a_{i}$. Since $U$ has only a finite number of faces of codimension 1, we may assume $g \in$ Aut $U$ to transform any face of codimension one into itself. However then we have $g^{*}\left(a_{i}{ }^{t} x\right)=a_{i}{ }^{t} g^{t} x=$ $\lambda_{i} a_{i}{ }^{t} x$ with a positive rational number $\lambda_{i}$. Hence $\lambda_{i}$ is an eigen-value of ${ }^{t} g \in G L(g Z)$ so that we have $\lambda_{i}=1$. By assumption there exist at least $N$ vectors $a_{i}$ independent over $\boldsymbol{R}$, therefore we conclude $g=$ identity.

Q.E.D.

Rroposition 1.2. Assume $\Sigma$ to be a $D-V$ cone in $\mathfrak{Y}_{g}^{+}$. Then Aut $\Sigma$ is finite. Moreover Aut $\Sigma \cap G L(3 Z)(\lambda)=1$ if $\lambda \geqq 3$.

Proof. The Delone decomposition w.r.t. $\Sigma\left(\Sigma \subset \mathfrak{Y}_{g}^{+}\right)$has at most a finite number of integral polyhedral subdivisions invariant by translations, hence in view of Lemma 1.1. $\Sigma$ is contained in at most a finite number of open $\mathrm{D}-\mathrm{V}$ cones. Aut $\Sigma$ induces a permutation on $\mathrm{D}-\mathrm{V}$ cones which contain $\Sigma$. According to Lemma 1.2., Aut $\Sigma^{\prime}$ is finite for an open $\mathrm{D}-\mathrm{V}$ cone $\Sigma^{\prime}$, because $\Sigma^{\prime}$ is contained in $\mathfrak{Y}_{g}^{+}$, so does not contain any line. Hence Aut $\Sigma$ is finite. $G L(g Z)(\lambda)$ contains no element of finite order distinct from identity if $\lambda \geqq 3$, so we are done.

Q.E.D.

(1.3) Let $B$ be a positive semi-definite symmetric half-integer $(g, g)$ matrix. Up to $G L(g Z)$ conjugate we may assume $B=\left(\begin{array}{cc}0 & 0 \\ 0 & B^{\prime \prime}\end{array}\right), B^{\prime \prime}$ $>0$. 
DeFinition 1.5. $S_{0}$ is a semigroup in $Z \times Z^{g}$ generated by $\left(m B^{t} m, m\right)$ $m \in Z^{g}$ and $\left(1,0_{g}\right)$.

LEMMA 1.3. (i) $S_{0}$ is finitely generated.

(ii) Assume the condition (A), then $S_{0}$ is saturated.

Proof. (i) is a consequence of ([13] p. 242).

(ii) It suffices to prove our lemma in case that $B$ is positive. We define a function $g$ by $g(m)=\operatorname{Min}\left\{N ;(N, m) \in S_{0}\right\}$. Obviously we have $g(m)=\operatorname{Min}\left\{\Sigma \ell_{j} m_{j} B^{t} m_{j} ; \ell_{j} \geqq 0, \Sigma \ell_{j} m_{j}=m, \ell_{j}, m_{j}\right.$ integral $\}$. And $(N, m)$ belongs to $S_{0}$ if and only if $N \geqq g(m)$. So it suffices to prove $g(r m)=$ $r g(m)$ for a positive integer. Assume $m \in \boldsymbol{R}^{+} \sigma$ for a Delone $g$ cell containing the origin. Let $\alpha$ be a vector satisfying the conditions $(0) \sim(2)$ for $\sigma$. We shall prove $g(m)=2 \alpha B^{t} m$. Assume $m=\Sigma \ell_{j} m_{j}$ with integral $\ell_{j}, m_{j}\left(\ell_{j} \geqq 0\right)$. Then we have $m_{j} B^{t} m_{j} \geqq 2 \alpha B^{t} m_{j}$, so that $\Sigma \ell_{j} m_{j} B^{t} m_{j} \geqq$ $2 \alpha B^{t}\left(\sum \ell_{j} m_{j}\right)=2 \alpha B^{t} m$. On the other hand, by the assumption (A) we can write $m=\Sigma r_{i} a_{i}$ with vertices $a_{i}$ of $\sigma$ and positive integers $r_{i}$. Since $\Sigma r_{j} a_{j} B^{t} a_{j}=2 \alpha B^{t}\left(\sum r_{j} a_{j}\right)=2 \alpha B^{t} m$, we have $g(m)=2 \alpha B^{t} m$, which completes the proof.

DEFINITION 1.6. $\mathrm{S}_{\Sigma, 0}$ is a semigroup in $\left(\overline{\mathfrak{Y}}_{g}\right)_{Z} \times Z^{g}$ generated by $(a, 0)$ $a \in(\check{\Sigma})_{Z},\left({ }^{t} m m, m\right) m \in Z^{g}$ where $\Sigma$ is a $\mathrm{D}-\mathrm{V}$ cone in $\overline{\mathfrak{Y}}_{g}^{+}$.

LEMMA 1.4. $S_{\Sigma, 0}$ is saturated and finitely generated under the assumption (A).

Proof. $(a, m) \in\left(\overline{\mathfrak{Y}}_{g}\right)_{Z} \times Z^{g}$ is contained in $S_{\Sigma, 0}$ if and only if for any $y \in \Sigma, \operatorname{tr}(a y)-2 \alpha y^{t} m \geqq 0$ with the notations in Lemma 1.3. Hence it is obvious that $S_{\Sigma, 0}$ is saturated. Since $(\check{\Sigma})_{Z}$ is finitely generated ([11] p. 7), the assertion follows.

LEMMA 1.5. In case of $g \leqq 4$. the conditions (A) and (B) hold.

Proof. Obviously the condition (A) holds true for any $\mathrm{D}-\mathrm{V}$ cone in $\mathfrak{Y}_{g}^{+}$if (A) holds for any open $\mathrm{D}-\mathrm{V}$ cones. According to Lemma 1.1, the condition (B) holds for $\Sigma^{\prime}$ if $\Sigma^{\prime}$ is a face of an open $\mathrm{D}-\mathrm{V}$ cone $\Sigma$ and (B) holds for $\Sigma$. In view of Voronoi's results, (A) and (B) hold for any open $\mathrm{D}-\mathrm{V}$ cone if $g \leqq 4$. ([26])

(1.4) We introduce a stronger condition (A*) than (A).

(A*) Let $a_{0}=0, a_{1}, \cdots, a_{g}$ be vertices of Delone $g$ cell $\sigma$ w.r.t. $B$. 
If $a_{1}, \cdots, a_{g}$ are linearly independent over $R$. then $a_{1}, \cdots, a_{g}$ form a basis of $Z^{g}$.

LEMMA 1.6. If the condition $\left(A^{*}\right)$ is satisfied by $B$, then $(A)$ is also satisfied.

Proof. Let a be contained in $\boldsymbol{R}^{+} \sigma \cap \boldsymbol{Z}^{g}$ where $\sigma$ is a Delone cell containing the origin. Then there exist $g$ vertices $a_{1}, \cdots, a_{g}$ linearly independent over $\boldsymbol{R}$ such that $a$ is contained in $\sum_{i=1}^{g} \boldsymbol{R}_{0}^{+} a_{i}$. $a$ is uniquely written as $a=\sum_{i=1}^{g} r_{i} a_{i}, r_{i} \geqq 0$. Since $a_{i}$ is a basis of $Z^{g}, a$ is also a linear combination of $a_{i}$ with integral coefficients.

Remark. If $g \leqq 3,\left(A^{*}\right)$ holds for any $B$. In case of $g=4$, many examples satisfy $\left(A^{*}\right)$. The author does not known any counter-example.

\section{§ 2. Stable quasi abelian varieties (SQAV)}

(2.1) We write a disc $D=\{s \in C ;|s|<\varepsilon\}, D^{*}=D-\{0\}, \mathcal{O}=\mathcal{O}_{0, D}=$ the local ring of holomorphic functions at the origin.

Let $\tau$ be a germ of a holomorphic mapping from $D^{*}$ to the Siegel upper half plane $\widetilde{\varsigma}_{g}$ of degree $g, \widetilde{S}_{g}=\{X+i Y ; X, Y$ real, symmetric, $Y>0\}$, surject to the unipotentcy condition,

$$
\tau\left(e^{2 \pi i} s\right)=\tau(s)+B
$$

with a positive semi-definite symmetric integral matrix $B$. Then we can write $\tau(s)=\tau_{0}(s)+B \log s / 2 \pi i$ with a holomorphic matrix $\tau_{0}(s)$ defined also at the origin.

It is well known that $\tau(s)(s \neq 0)$ determines a principally polarized abelian variety in a canonical manner.

The sympletic group $S p(g Z)$ operates on $\mathfrak{S}_{g}$ by,

$$
M=\left(\begin{array}{ll}
A & B \\
C & D
\end{array}\right): \tau \rightarrow(A \tau+B)(C \tau+D)^{-1}, \quad M \in S p(g Z)
$$

then $\tau$ and $(A \tau+B)(C \tau+D)^{-1}$ determine isomorphic principally polarized abelian varieties.

Definition 2.1. $R=\mathcal{O}\left[e\left(\frac{1}{2} m \tau\left(s^{2}\right)^{t} m+m^{t} \zeta\right) \theta, m \in Z^{g}\right]$, where $\theta$ is an indeterminate. Here we consider $R$ as an $\mathcal{O}$ graded ring by, 
$\operatorname{deg} \theta=1, \quad \operatorname{deg}\left(e\left(\frac{1}{2} m \tau\left(s^{2}\right)^{t} m+m^{t} \zeta\right)\right)=0, \quad \operatorname{deg} a=0 \quad(a \in \mathcal{O})$.

Proposition 2.1. (i) Proj $R$ is a locally noetherian analytic space with a canonical projection $\widetilde{\sigma}$ to $\mathrm{Spec} \mathcal{O}$.

(ii) Proj $R$ is covered with open affines $U_{k}=\operatorname{Spec} R_{k}, k \in Z^{g}$ where $R_{k}=\mathcal{O}\left[e\left(\frac{1}{2} m \tau\left(s^{2}\right)^{t} m-\frac{1}{2} k \tau\left(s^{2}\right)^{t} k+(m-k)^{t} \zeta\right), m \in Z^{g}\right]$

(iii) Under the assumption (A), Proj $R$ is normal, Cohen-Macaulay and $\varpi$ is flat, reduced, Cohen-Macaulay.

Proof. (i) (ii) is obvious from the definition and (i) of Lemma 1.3. (iii) Since $R_{k} \cong R_{0}$, we suffices to prove $R_{0}$ is normal, Cohen-Macaulay and $\left.\widetilde{\sigma}\right|_{U_{0}}$ is flat reduced, Cohen-Macaulay. Up to $G L(g Z)$ conjugate, we may assume $B=\left(\begin{array}{cc}0 & 0 \\ 0 & B^{\prime \prime}\end{array}\right), B^{\prime \prime}>0$, rank $B^{\prime \prime}=g^{\prime \prime}, g=g^{\prime}+g^{\prime \prime}$. Then we have

$$
\begin{aligned}
R_{0} & =\mathcal{O}\left[s^{m B^{t} m} e\left(m^{t} \zeta\right), m \in Z^{g}\right] \\
& =\mathcal{O}\left[s^{m^{\prime \prime} B^{\prime \prime} t} e\left(m^{t} \zeta\right), m=\left(m^{\prime}, m^{\prime \prime}\right) \in Z^{g}\right] \\
& =\mathcal{O}\left[e\left(m^{\prime t} \zeta^{\prime}\right), s^{m^{\prime \prime} B^{\prime \prime} m^{\prime \prime}} e\left(m^{\prime \prime t} \zeta^{\prime \prime}\right), m^{\prime} \in Z^{g^{\prime}}, m^{\prime \prime} \in Z^{g^{\prime \prime}}\right] \quad\left(\zeta=\left(\zeta^{\prime}, \zeta^{\prime \prime}\right)\right)
\end{aligned}
$$

The normality and Cohen-Macaulayness of $R_{0}$ follow from the saturatedness of a semigroup $S_{0}$ associated with $B^{\prime \prime}$. ([11] p. 5 and p. 52) However $S_{0}$ is saturated in view of Lemma 1.3. Next we turn to the proof of the second assertion. Assume $\varpi$ not to be reduced, then we have an element $f$ of $R_{0}$ such that $f^{r}=s h$, with $h \in R_{0}, f \notin s R_{0}$. Viewing that $R_{0}$ is a graded ring in $s, e\left(\zeta_{1}\right) \cdots, e\left(\zeta_{g}\right)$, we may assume $f$ is a monomial, say, $s^{a} e\left(b^{t} \zeta\right)$. Then $\left(s^{a} e\left(b^{t} \zeta\right)\right)^{r}=s h$ implies $r a>g(r b)$. Since $f \notin s R_{0}$, $a=g(b)$, which contradicts to the fact $g(r b)=r g(b)$. Since Proj $R$ is Cohen-Macaulay and $\widetilde{\sigma}$ is obviously equidimensional (see 2.3), so $\widetilde{\sigma}$ is flat. We shall prove later that $\varpi$ is Cohen-Macaulay.

(2.2) Next we shall construct a troidal embedding corresponding to Proj $R$. This construction is one parameter case of Namikawa [18], and of course the idea is quite the same as his.

Definition 2.2. A subset $\Delta$ of $\boldsymbol{R}_{0}^{+} \times \boldsymbol{R}^{g} B$ is called a mixed V-cone with respect to $B$ if

$$
\Delta=\text { interior of } \Delta_{k} \text { or a face of } \Delta_{k} \quad\left(k \in Z^{g}\right)
$$

where $\Delta_{k}=\left\{(y, x) \in \boldsymbol{R}_{0}^{+} \times \boldsymbol{R}^{g} ;\left(m B^{t} m-k B^{t} k\right) y+(m-k)^{t} x \geqq 0, m \in \boldsymbol{Z}^{g}\right\}$. 
Remark. $\{\Delta\}$ forms a convex rational polyhedral decomposition of $\boldsymbol{R}_{0}^{+} \times \boldsymbol{R}^{g}$, which we call mixed Voronoi decomposition of $\boldsymbol{R}_{0}^{+} \times \boldsymbol{R}^{g}$.

LEMMA 2.1. Under the assumption $(A), S_{0}=\left(\check{\Delta}_{0}\right)_{Z}=\left\{(a, m) \in Z_{0}^{+} \times \boldsymbol{Z}^{g}\right.$; $a y+m^{t} x \geqq 0$ for any $\left.(y, x) \in \Delta_{0}\right\}$.

Proof. It suffices to prove $\check{S}_{0}=\Delta_{0}$ since $S_{0}$ is saturated. (lemma 1.3.) However this is obvious from the definition.

From Lemma 2.1., it follows by the theory of troidal embeddings [11],

Proposition 2.2. Under the assumption (A), Proj $R$ is a troidal embedding associated with a mixed Voronoi decomposition of $\boldsymbol{R}_{0}^{+} \times \boldsymbol{R}^{g}$.

(2.3) Proposition 2.3. We assume the condition $(A)$ to be satisfied for $B>0$. Let $P=$ Proj $R, P_{0}=$ the closed fiber of $P$ by $\varpi$. Then $P_{0}$ is described as follows,

(i) $P_{0}$ is covered with open affine sets $\left(U_{k}\right)_{0}, k \in Z^{g}$.

(ii) each $\left(U_{k}\right)_{0}$ is isomorphic to $\left(U_{0}\right)_{0}$,

$$
\left(U_{k}\right)_{0}=\operatorname{Spec} C\left[x_{a}^{(k)}, a \in \Phi\right], \quad x_{a}^{(k)}=s^{a B^{t} a+2 k B^{t} a} e\left(a^{t} \zeta\right)
$$

where $\Phi$ denotes the set of all vertices of Delone 1-cells containing the origin.

(iii) the fundamental relations between $x_{a}, a \in \Phi$ are given by

$$
\begin{aligned}
\prod_{i \in I} x_{a_{i}}= & 0 \text { if no Delone } g \text { cell contains all } a_{i} \quad i \in I . \\
\prod_{i \in I} x_{a_{i}}= & \prod_{i \in I^{\prime}} x_{a_{i}} \text { if } a_{i}\left(i \in I \cup I^{\prime}\right) \text { are contained in a Delone } \\
& g \text { cell } \sigma \text { and moreover } \sum_{i \in I} a_{i}=\sum_{i \in I^{\prime}} a_{i} .
\end{aligned}
$$

(iv) $\left(U_{0}\right)_{0}$ is a union of $Z_{\tau}(\tau$ : Delone cell containing the origin)

$$
\begin{array}{ll}
Z_{\tau} \cong \operatorname{Spec} C\left[x_{a}, x \in \tau\right] & x_{a}=x_{a}^{(0)} \\
Z_{\sigma_{1}} \cap Z_{\sigma_{2}}=Z_{\sigma_{1} \cap \sigma_{2}}, & Z_{\tau} \subset Z_{\sigma} \text { if } \tau \prec \sigma
\end{array}
$$

(v) $\left(U_{0}\right)_{0} \cap\left(U_{k}\right)_{0} \neq \phi$ if and only if $k \in \Phi$

(vi) Given a Delone $g$ cell $\sigma$, the structure of the closure $\bar{Z}_{\sigma}$ of $Z_{\sigma}$ in $P$, is described as follows. For a vertex $a_{0}$ of $\sigma$ we define $Y_{a_{0}}=$ Spec $C\left[s^{a B^{t} a-a_{0} B^{t} a_{0}} e\left(\left(a-a_{0}\right)^{t} \zeta\right), a \in \sigma\right]$ and $\Delta^{\prime}\left(a_{0}\right)=\left\{x-x_{0} ; x \in \Delta\left(\left\{a_{0}\right\}\right), x_{0} \in \Delta(\sigma)\right\}$ where $\Delta(\tau)$ denotes a Voronoi cell corresponding to $\tau$. Let $C_{a_{0}}=R^{+} \Delta^{\prime}\left(a_{0}\right)$ $=\left\{\lambda y ; y \in \Delta^{\prime}\left(a_{0}\right), \lambda>0\right\}$. Then $\overline{\mathscr{C}}=\left\{C_{a_{0}}, a_{0} \in \sigma\right.$ and a face of $\left.C_{a_{0}}\right\}$ gives $a$ 
rational convex conical decomposition of $\boldsymbol{R}^{g}$ and $\bar{Z}_{\sigma}$ is an equivariant completion of an algebraic torus $\left(C^{*}\right)^{g}$ w.r.t. $\overline{\mathscr{C}}$. ([11], [21])

Proof. (i) and (ii) are direct consequences of the definition and the proof of Lemma 1.3 .

(iii) If all $a_{i}$ are contained in the same $\sigma$ and $\sum_{i \in I_{1}} a_{i}=\sum_{i \in I_{2}} a_{i}$, then we have for some $\alpha \in \boldsymbol{R}^{g}, a_{i} B^{t} a_{i}=2 \alpha B^{t} a_{i} i \in I_{1} \cup I_{2}$, hence $g\left(\sum_{i \in I_{\nu}} a_{i}\right)$ $=\sum_{i \in I_{\nu}} a_{i} B^{t} a_{i}=2 \alpha B^{t}\left(\sum_{i \in I_{\nu}} a_{i}\right), \nu=1,2$. Thus we obtain $\prod_{i \in I_{1}} x_{a_{i}}=$ $\prod_{i \in I_{2}} x_{a_{i}}$. If there's no Delone cell containing all $a_{i}, i \in I$, at the same time, then $g\left(\sum_{i \in I} a_{i}\right)<\sum a_{i} B^{t} a_{i}$. In fact, assume the contrary. Take a Delone cell $\sigma$ such that $m=\sum_{i \in I} a_{i} \in \boldsymbol{R}^{+} \sigma$. Then there exisits $\alpha \in \boldsymbol{R}^{g}$ such that $a B^{t} a \geqq 2 \alpha B^{t} a$, equality holds if and only if $a \in \sigma$. In view of Lemma 1.3, $g(m)=2 \alpha B^{t} m=\sum_{i \in I} 2 \alpha B^{t} a_{i}$. If $g(m)=\sum a_{i} B^{t} a_{i}$, then $a_{i} \in \sigma(i \in I)$ since $a_{i} B^{t} a_{i}=2 \alpha B^{t} a_{i}(i \in I)$, which is a contradiction.

(iv) is obvious from (iii).

(v) If $k \in \Phi$, then it is obvious that $\left(U_{0}\right)_{0} \cap\left(U_{k}\right)_{0} \neq \Phi$. Conversely assume $\left(U_{0}\right)_{0} \cap\left(U_{k}\right)_{0} \neq \phi$. Then there exists a point $p$ such that $x_{k}(p) \neq 0$, $x_{k}=s^{k B^{t} k} e\left(k^{t} \zeta\right) \in \Gamma\left(\left(U_{0}\right)_{0}\right)$.

This implies $g(k)=k B^{t} k$, i.e. $k=a_{i}$ for some $i$.

(iv) With loss of generality, we may assume $a_{0}=0$. We have already known

$$
\begin{aligned}
\Delta(\{0\}) & =\left\{x ; a B^{t} a+a^{t} x \geqq 0,\right. & & \text { for any } \left.a \in Z^{g}\right\} \\
& =\left\{x ; a B^{t} a+a^{t} x \geqq 0,\right. & & \text { for any } a \in \Phi\}
\end{aligned}
$$

and $\Delta(\sigma)=\left\{x_{0}\right\}=\left\{x ; a_{i} B^{t} a_{i}+a_{i}{ }^{t} x=0\left(a_{i} \in \sigma\right), a B^{t} a+a^{t} x \geqq 0\right.$ for any $\left.a \in Z^{g}\right\}$. In order to prove the assertion, we suffices to show that $Y_{a_{0}}$ is an equivalent embedding of algebraic torus associated with $C_{a_{0}}$. For that purpose, it is sufficient to show that $C_{a_{0}}=\left(\boldsymbol{R}^{+} \sigma\right)^{\vee}=\left\{x ; x^{t}\left(a-a_{0}\right) \geqq 0\right.$, for any $a \in \sigma\} . \quad\left(a_{0}=0\right)$ If $x-x_{0} \in \Delta^{\prime}\left(a_{0}\right),\left(x \in \Delta\left(\left\{a_{0}\right\}\right), x_{0} \in \Delta(\sigma)\right)$, then

$$
\left(x-x_{0}\right)^{t} a_{i}=x^{t} a_{i}-x_{0}{ }^{t} a_{i} \geqq-a_{i} B^{t} a_{i}+a_{i} B^{t} a_{i}=0,
$$

so that we have $C_{a_{0}} \subset\left(\boldsymbol{R}^{+} \sigma\right)^{\vee}$. Conversely let $x^{\prime}$ be contained in $\left(\boldsymbol{R}^{+} \sigma\right)^{\vee}$, i.e., $x^{\prime t} a_{i} \geqq 0$ for any $a_{i} \in \sigma$. Put $N=\operatorname{Min}_{a \neq a_{i}, 0}\left\{a B^{t} a+a^{t} x_{0}\right\}$. Obviously $N>0$. Take a positive number $r$ such that $\left|a^{t} x^{\prime}\right|<r N$, for any $a \in \Phi$ $a \neq a_{i}, 0$. Put $x=\left(x^{\prime} / r\right)+x_{0}$. Then we have, $a_{i} B^{t} a_{i}+a_{i}{ }^{t} x=a_{i} B^{t} a_{i}+$ $a_{i}{ }^{t} x_{0}+\left(a_{i}{ }^{t} x^{\prime} / r\right) \geqq 0\left(a_{i} \in \sigma\right) a B^{t} a+a^{t} x=a B^{t} a+a^{t} x_{0}+\left(a^{t} x^{\prime} / r\right) \geqq\left(a^{t} x^{\prime} / r\right)$ $+N>0\left(a \in \Phi, a \neq a_{i}, 0\right)$ so that $x$ is contained in $C_{a_{0}}$. Hence we obtain 
$x^{\prime}=r\left(x-x_{0}\right), x \in C_{a_{0}}, x_{0} \in \Delta(\sigma)$.

Q.E.D.

(2.4) Now we define an action of a lattice $\Gamma\left(=Z^{g}\right)$ on $P$ and construct a quotient space $\mathscr{A}=P / \lambda \Gamma, \lambda \Gamma=\{\lambda n ; n \in \Gamma\}, \lambda \in Z^{+}$. For an element $n$ of $\Gamma$ we define $\mathcal{O}$-endomorphism of $R$ by,

$$
\begin{gathered}
S_{n}^{*}(a)=a, \quad a \in \mathcal{O} \\
S_{n}^{*}\left(e\left(\frac{1}{2} m \tau^{t} m+m^{t} \zeta\right) \theta\right)=e\left(\frac{1}{2}(m+n) \tau^{t}(m+n)+(m+n)^{t} \zeta\right) \theta .
\end{gathered}
$$

Thus $S_{n}$ transforms $U_{k}$ into $U_{k+n}$.

In order to prove that $\Gamma$ acts on $P$ properly discontinuously we take a local analytic model $P$ over $D_{\varepsilon}=\{s \in C ;|s|<\varepsilon\}$, a local representative $\tau$ defined over $D_{\varepsilon}$ for a sufficiently small $\varepsilon>0$. Our aim is to show that for any point $p$ in $P_{0}$, there exists a small neighborhood $U(p, \varepsilon)$ such that $S_{n}(U(p, \varepsilon)) \cap U(p, \varepsilon)$ is empty except at most a finite number of $n$. For brevity we assume the condition $(A)$ is satisfied and $B>0$. Moreover assume that $Z_{\sigma}$ is the minimal subvariety containing $p$ where $\sigma$ is a Delone cell containing the origin. Then we may take a neighborhood $U(p, \varepsilon)$ of $p$ defined by, $U(p, \varepsilon)=\left\{\left(s, x_{a}\right) a \in \Phi ;|s|<\varepsilon_{1},\left|x_{a}\right|<\varepsilon_{2}(a \notin \sigma)\right.$, $\left.\left|x_{a}-x_{a}(p)\right|<\varepsilon_{3}(a \in \sigma)\right\} \varepsilon=\left(\varepsilon_{1}, 3_{2}, \varepsilon_{3}\right)$. Notice that $x_{a}(p) \neq 0(a \in \sigma)$.

Assume $S_{n}(U(p, \varepsilon)) \cap U(p, \varepsilon) \neq \phi$. Take a point $x^{0}$ from $S_{n}(U(p, \varepsilon)) \cap$ $U(p, \varepsilon)$, then we may assume $x^{0}$ to be given by $\left(s_{0}, x_{a}^{0}\right),\left(s_{0} \neq 0\right)$ with finite exception of $n$. (Recall that $\left(U_{0}\right)_{0} \cap\left(U_{k}\right)_{0} \neq \phi$ if and only if $k=a_{i}$ for some i.) Hence we have,

$$
\begin{aligned}
& 0<\left|s_{0}\right|<\varepsilon_{1}, \quad\left|x_{a}^{0}\right|<\varepsilon_{2} \quad\left|x_{a}^{0}-x_{a}(p)\right|<\varepsilon_{3} \\
& (a \notin \sigma) \\
& \left|s^{a B^{t} n} x_{a}^{0}\right|<\varepsilon_{2} \quad\left|s^{a B^{t} n} x_{a}^{0}-x_{a}(p)\right|<\varepsilon_{3} \\
& (a \in \sigma)
\end{aligned}
$$

Taking at first $\varepsilon_{2}, \varepsilon_{3}$ in such a way that $0<\varepsilon_{2} \ll 1,0<\varepsilon_{3} \ll \operatorname{Min}_{a \epsilon_{\sigma}}\left|x_{a}(p)\right|$ and then $\varepsilon_{1} \ll \operatorname{Min}\left(\varepsilon_{2}, \varepsilon_{3}\right)$, we have

$$
\begin{aligned}
& \left|s_{0}^{2 a B^{t} a+a B^{t_{n}}}\right|=\left|s_{0}^{a B^{t} n} x_{a}^{0}\right|\left|x_{-a}^{0}\right|<1 \\
& \left|s_{0}^{2 a B^{t} a-a B^{t} n}\right|=\left|s_{0}^{-a B^{t} n} x_{-a}^{0}\right|\left|x_{a}^{0}\right|<1
\end{aligned}
$$

if $a \notin \sigma$ or $-a \notin \sigma$, so that $2 a B^{t} a+a B^{t} n>0,2 a B^{t} a-a B^{t} n>0$. On the other hand $\sigma$ does not contain both $a$ and $-a$, i.e., $a \notin \sigma$ or $-a \notin \sigma$. Hence $S_{n}(U(p, \varepsilon)) \cap U(p, \varepsilon)=\phi$ except a finite number of $n$. Even in the general case, since $R_{0}$ is finitely generated, it is easy to check the action of $\Gamma$ on $P$ is properly discontinuous and free along the same line of arguments as above. Thus we have proved that the action of $\Gamma$ on $P$ is 
properly discontinuous and obviously free. (see footnote.)

On the other hand $S_{n}^{*} S_{m}^{*}=S_{m}^{*} S_{n}^{*}=S_{m+n}^{*}$ by the symmetry of $\tau$, so $0(1)$ on $P$ can be descended to an invertible sheaf which is also denoted by $0(1)$. Then we have,

Proposition 2.4. (i) The quotient space $P / \lambda \Gamma$ exists and has a canonical proper projection $\varpi$ to $\operatorname{Spec} \mathcal{O}$.

(ii) (Namikawa) Under the assumption (A), 0(1) is relatively ample on $P / \lambda \Gamma$

(iii) Under the assumption (A), $\widetilde{x}$ is a reduced, flat, Cohen-Macaulay morphism. $P / \lambda \Gamma$ is normal, Cohen-Macaulay.

Proof. The proof of (ii) should be refered to Namikawa [18]. The other assertions follow from Proposition 2.1..

Next we investigate a section of $P$ over Spec $\mathcal{O}$ under the assumption (A) and define a concept of level $\lambda$-structure on $P / \lambda \Gamma$.

Let $\tau=\tau_{0}(s)+B \log s / 2 \pi \sqrt{-1},{ }^{t} \tau=\tau, B=\left(\begin{array}{cc}0 & 0 \\ 0 & B^{\prime \prime}\end{array}\right), B^{\prime \prime}>0$. Take a Delone $g^{\prime \prime}$-cell $\sigma$ w.r.t. $B^{\prime \prime}$ containing the origin. Set $g^{\prime}=g-g^{\prime \prime}$.

Let $a_{j}(j=0, \cdots, r)$ be all the vertices of $\sigma, a_{0}=0$. Then by definition there exists a vector $\alpha\left(\in \boldsymbol{R}^{g^{\prime \prime}}\right)$ such that $a_{j} B^{t} a_{j}=2 \alpha B^{t} a_{j}(j=1, \cdots, r)$. By assumption $(A), 2 \alpha B$ is an integral vector because $\left\{a_{j}\right\} \operatorname{span} R^{+} \sigma \cap Z^{g^{\prime \prime}}$ over $\boldsymbol{Z}_{0}^{+}$, so $\boldsymbol{Z}^{g^{\prime \prime}}$ over $\boldsymbol{Z}$ where $\boldsymbol{Z}_{0}^{+}$denotes nonnegative integers.

Then we define an $\mathcal{O}$-algebra $R^{*}=R^{*}(\tau)$ by,

$$
R^{*}(\tau)=\mathcal{O}\left[\xi_{m}^{*} \cdot \theta m \in Z^{g}\right], \quad \xi_{m}^{*}=e\left(\frac{1}{2} m \tau_{0}\left(s^{2}\right)^{t} m+m^{t} \zeta\right) s^{m B^{t} m-2 \alpha B^{t}} .
$$

$R^{*}(\tau)$ is obviously isomorphic to $R(\tau)$ as $\mathcal{O}$-algebras. $\Gamma$ operates on $\operatorname{Proj} R^{*}(\tau)$ properly discontinuously and free and $\operatorname{Proj} R^{*}(\tau) / \lambda \Gamma$ exists. Since $\left.B=\left(\begin{array}{cc}0 & 0 \\ 0 & B^{\prime \prime}\end{array}\right), R^{*}(\tau)=\mathcal{O}\left[\xi_{e_{j}}^{*}\right)^{ \pm 1}, \xi_{0 m_{2}}^{*}\right]\left(e_{j} ; j\right.$-th unit vector, $\left.m_{2} \in Z^{g^{\prime \prime}}\right)$.

We define a section $\varepsilon_{\sigma}$ by $\xi_{e_{j}}^{*}=1, \xi_{0 a_{j}}^{*}=1\left(a_{j} \in \sigma\right)$ and $\varepsilon_{o+n}$ by $\xi_{e_{j+n}}^{*}=$ $\xi_{0 a_{j+n}}^{*}=1, \varepsilon_{\sigma+n}\left(n \in Z^{g}\right)$ is a section not only of $P^{*}=\operatorname{Proj} R^{*}(\tau)$ but also of $P^{*} / \lambda \Gamma$. On $P^{*} / \lambda \Gamma, \varepsilon_{\sigma+n}=\varepsilon_{\sigma+n^{\prime}}$ if and only if $n \equiv n^{\prime} \bmod \lambda$. On the other hand $\hat{G}_{\sigma}=\operatorname{Spec} \mathcal{O}\left[\left(\xi_{e_{j}}^{*}\right)^{ \pm 1}, \xi_{0 a_{j}}^{*}\right]$ is an $\mathcal{O}$-group scheme w.r.t. the usual multiplication of a split torus with unity $\varepsilon_{\sigma}$, and hence the image $G_{\sigma}$ of $\hat{G}_{\sigma}$ in $P^{*} / \lambda \Gamma$ is an open subscheme and also an $\mathcal{O}$-group scheme. The union $G$ of all translations of $G_{\sigma}$ by $\Gamma$ forms a $\mathcal{O}$-group scheme and an open subscheme of $P^{*} / \lambda \Gamma$. Let $e_{j}=\sum_{k=1}^{r} n_{j k} a_{k},\left(e_{j}=j\right.$-th unit, $g^{\prime}+1 \leqq$

This proof is valid only in the case $\tau_{0}=0, B>0$. In a more general set-up we can prove the existence of quotients ([15] §2). 
$\left.j \leqq g, n_{j k} \in Z\right)$, and $\eta_{e_{j}}=\xi_{e_{j}}^{*}\left(1 \leqq j \leqq g^{\prime}\right), \eta_{e_{j}}=\prod\left(\xi_{a_{k}}^{*}\right)^{n_{j k}}\left(g^{\prime}+1 \leqq j \leqq g\right)$.

Put

$$
\begin{array}{ll}
\varepsilon_{\nu}: \eta_{e_{j}}=1, \quad(j \neq \nu) & \eta_{e_{\nu}}=e^{2 \pi i / \lambda} \quad(1 \leqq \nu \leqq g) \\
\varepsilon_{\nu}: \eta_{e_{j}}=e\left(e_{\nu} \tau_{0}{ }^{t} e_{j}\right) & \left(g+1 \leqq \nu \leqq g+g^{\prime \prime}\right) \\
\varepsilon_{\nu}=\varepsilon_{\sigma+e_{\nu}} & \left(g+g^{\prime \prime}+1 \leqq \nu \leqq 2 g\right)
\end{array}
$$

Then $\varepsilon_{\nu} 1 \leqq \nu \leqq 2 g$ forms a basis of the set of all $\lambda$-division points of $G$. Obviously $\varepsilon_{\nu}(g+1 \leqq \nu \leqq 2 g)$ operates on $0(1)$ invariantly.

Let $H$ be the set of all $\lambda$-division points of $G$ and $H^{\prime}$ be the subgroup scheme generated by $\varepsilon_{\nu}(g+1 \leqq \nu \leqq 2 g)$.

Definition 2.3. $\xi=\left(P^{*} / \lambda \Gamma, \widetilde{\varpi}\right.$, Spec $\left.\mathcal{O}, G, H, H^{\prime}, \varepsilon_{j}, 0(1)\right)$ is called a principally polarized stable quasi-abelian variety (or scheme) with level $\lambda$-structure over Spec $\mathcal{O}$. (or abbr. SQAV or SQAS) The closed fiber of $\xi$ is called a SQAV (SQAS) over $C$.

Notice that our definition of level structure depends on the choice of a Delone cell $\sigma$. Although Namikawa has defined this by the action of $\Gamma \bmod \lambda \Gamma$, it seems that our definition is better in order to prove the existence of moduli ( $\S 6)$.

Next for later use $(\S 6)$ we shall define over any algebraically closed field $K($ char $K=0)$ a principally polarized stable quasi abelian variety.

Let $R=K[[t]]\left[\xi_{m} \cdot \theta, m \in Z^{g}\right], \xi_{m}=\left(\prod_{i, j} a_{i j}^{m_{i j} m_{j}}\right) t^{m B^{t} m} w^{m}, B$ : a positive semi difinite matrix, ${ }^{t} B=B, a_{i j} \in K, a_{i j}=a_{j i}$. We can define an action of $\Gamma\left(=Z^{g}\right)$ in a similar manner. We assume that an action of $\Gamma$ is free and properly discontinuous, and Proj $R / \lambda \Gamma$ exists as a projective $K[[t]]$-scheme with an ample invertible sheaf $0(1)$.

Moreover we assume here the condition (A) for $B$ holds. Then by the same argument as above, an open subscheme $G$, sections $\varepsilon_{j}, H$ and $H^{\prime}$ are defined.

Definition 2.4. $\left(P / \lambda \Gamma, \widetilde{\sigma}, \operatorname{Spec} K[[t]], G, H, H^{\prime}, \varepsilon_{j}, 0(1)\right) \times_{K[[t]]} \operatorname{Spec} K$ is called a principally polarized stable quasi abelian variety level $\lambda$ structure over $K$.

In the case where $K=C$, we may assume $B=\left(\begin{array}{cc}0 & 0 \\ 0 & B^{\prime \prime}\end{array}\right), B^{\prime \prime}>0$, ${ }^{t} B^{\prime \prime}=B^{\prime \prime}$, and $a_{i j}=e\left(\frac{1}{2}\left(\tau_{0}\right)_{i j}\right)$. The existence of the quotient $(P / \lambda \Gamma), 0(1)$ implies that the principal $\left(g-g^{\prime \prime}\right)$-minor $\tau_{0}^{1}$ of $\tau_{0}$ gives an abelian variety. We may assume then with loss of generality $\tau_{0}^{1}$ is contained in the Siegel upper half plane of degree $\left(g-g^{\prime \prime}\right)$. So our second definition of SQAV 
is the same as the first one in case of $K=C$.

Remark. We give another construction of SQAV for later use. Let $\tau$ be a period matrix as before, $\tau=\tau_{0}(s)+B \log s / 2 \pi \sqrt{-1},{ }^{t} B=B \geqq 0$. We define $\tilde{R}(\tau)=\mathcal{O}\left[\tilde{\xi}_{m} \cdot \theta\right], \tilde{\xi}_{m}=e\left(\frac{1}{2} m \tau_{0}(s)^{t} m+m^{t} \zeta\right) s^{1 / 2\left(m B^{t} m-r^{t} m\right)}$ where $r$ denotes the diagonal vector of $B$ i.e. $r_{i}=b_{i i}, B=\left(b_{i j}\right) . \quad \Gamma\left(=Z^{g}\right)$ acts on $\tilde{P}=\operatorname{Proj} \tilde{R}(\tau)$ properly discontinuously and freely. The closed fibers of $\tilde{P}, \tilde{P} / \lambda \Gamma$ are the same as before. Se we omit details.

(2.5) Let $X$ be a SQAV, and $B$ be a positive semi definite matrix, ${ }^{t} B=B, B=\left(\begin{array}{cc}0 & 0 \\ 0 & B^{\prime \prime}\end{array}\right), B^{\prime \prime}>0, B^{\prime \prime} \in M_{g^{\prime \prime}}(Z)$. We consider the Delone decomposition associated with $B$. Let $\sigma_{k}$ be a Delone cell of dimension $k$ of this decomposition $(0 \leqq k \leqq g)$. In what follows, we fix an orientation of $\sigma$ in such a way that every pair of adjacent $g$ cells have converse orientations to each other along their common faces.

LEMMA 2.2. Assume the condition (A) for $B$. (or in this case we say simply $X$ satisfies the condition $(A)$ ). With the notations in 2.3 ) we have an exact sequence

$$
0 \longrightarrow \mathcal{O}_{P_{0}} \stackrel{\iota^{*}}{\longrightarrow} \oplus \mathcal{O}_{Z \sigma_{g}} \stackrel{\psi_{0}}{\longrightarrow} \oplus \mathcal{O}_{Z \sigma_{g-1}} \stackrel{\psi_{1}}{\longrightarrow} \cdots \stackrel{\psi_{g^{\prime \prime}-1}}{\longrightarrow} \oplus \mathcal{O}_{Z_{\sigma_{g-g^{\prime \prime}}}} \longrightarrow 0
$$

where $\mathcal{O}_{Y}$ denotes the structure sheaf of $Y, g^{\prime \prime}=\operatorname{rank} B$ and $\iota^{*}$ is $a$ homomorphism induced by the normalization of $P_{0} . \quad \psi_{k}$ is defined by

$$
\psi_{k}\left(\left(a_{\sigma}\right)\right)=\left(b_{\tau}\right), \quad b_{\tau}=\sum_{\tau \prec \sigma}[\sigma: \tau] a_{\sigma} .
$$

Proof. Since each of $P_{0}, Z_{\sigma}$ is reduced, the exactness follows from that of the usual topological resolution

$$
0 \rightarrow Q\left(P_{0}\right) \rightarrow \oplus Q\left(Z_{\sigma}\right) \rightarrow \cdots \rightarrow \oplus Q\left(Z_{\sigma_{g-g^{\prime \prime}}}\right) \rightarrow 0
$$

where $Q(Y)$ denote the constant sheaf $Q$ of rational numbers on $Y$.

Let $X=P_{0} / \lambda \Gamma, \tilde{X}=$ the normalization of $X$. Denote by $X_{\sigma}$ the inverse image of $Z_{\sigma}$ in $\tilde{X}$. Then we have

COROLlaRY 2.1. Under the assumption (A) we have,

$$
0 \rightarrow \mathcal{O}_{X} \rightarrow \underset{\sigma \bmod \lambda \Gamma}{\bigoplus_{X}} \mathcal{O}_{\sigma_{\sigma}} \rightarrow \underset{\sigma \bmod \lambda \Gamma}{\bigoplus_{X \sigma_{g-1}}} \mathcal{O}_{\sigma^{\prime}} \rightarrow \cdots \rightarrow \underset{\sigma \bmod \lambda \Gamma}{\bigoplus_{X}} \mathcal{O}_{X_{\sigma-g^{\prime \prime}}} \rightarrow 0
$$

COROLLARY 2.2. Under the assumption $(A)$ we have 
(i) $\operatorname{dim} H^{i}\left(X \mathcal{O}_{X}\right)=\left(\begin{array}{l}g \\ i\end{array}\right)$

(ii) a canonical homomorphism $H^{1}(X C) \rightarrow H^{1}\left(X \mathcal{O}_{X}\right)$ is surjective.

Proof. (i) Assume $g=g^{\prime \prime}$, i.e. $B>0$. Since $H^{i}\left(X_{\sigma}, \mathcal{O}_{X_{\sigma}}\right)=0 i>0$ by the general theory ([11] p. 44), it follows from the above Corollary that

$$
\begin{aligned}
H^{i}\left(X \mathcal{O}_{X}\right) & =H^{i}\left(\left\{\oplus H^{0}\left(X_{\sigma}, \mathcal{O}_{X_{o}}\right)\right\}\right) \\
& =H^{i}\left(\left\{\underset{\sigma}{\oplus} \boldsymbol{C}_{\sigma}\right\}\right) \\
& =H^{i}\left(\boldsymbol{R}^{g} / \boldsymbol{Z}^{g}, C\right)=C^{\left(\frac{q}{i}\right)} .
\end{aligned}
$$

In the general case, noting that $X_{\sigma}$ is a fiber bundle over a fixed abelian variety $A$ with fiber $X_{\sigma}^{\prime}$ where $X_{\sigma}^{\prime}$ is a variety corresponding to $\sigma$ w.r.t. $B^{\prime \prime}, B=\left(\begin{array}{cc}0 & 0 \\ 0 & B^{\prime \prime}\end{array}\right)$, we infer easily our Corollary.

(ii) is obvious from the above proof.

Remark. Let $B=\left(\begin{array}{cc}0 & 0 \\ 0 & B^{\prime \prime}\end{array}\right)$ rank $B^{\prime \prime}=g^{\prime \prime} . \quad$ Then we have $H^{1}(X Z)=$ $Z^{2 g-g^{\prime \prime}}$ by the same argument noting that $X_{\sigma}^{\prime}$ is simply connected.

Corollary 2.3. Under the assumption (A), $X$ is Cohen Macaulay.

Proof. According to Proposition 2.3 (vi) ([11] p. 52) any $X_{\sigma}$ is Cohen-Macaulay, or equivalently $\mathscr{H}_{x_{0}}^{i}\left(0_{x_{\sigma}}\right)=0\left(i<\operatorname{dim} X_{o}, x_{0}^{\prime} \in X_{\sigma}\right)$. Using Corollary 2.1 we can prove $\mathscr{H}_{x_{0}}^{i}\left(0_{X}\right)=0\left(i<g, x_{0} \in X\right)$ inductively.

(2.5) Next under the assumption (A) we shall give an explicit description of $\mathscr{E}_{x t}{ }^{0}\left(\Omega_{X}^{1} \mathcal{O}_{X}\right)$ and moreover compute $\operatorname{dim} \operatorname{Ext}^{0}\left(\Omega_{X}^{1} \mathcal{O}_{X}\right)$ in the case where $\lambda=1$ and the condition (B) holds for $X$. Assume $B>0$, consider the Delone decomposition associated with $X$, i.e., $B$. Let $a_{i}$ be an extremity distinct from the origin of a Delone 1-cell w.r.t. $B$ containing the origin. We put $\Phi=$ the set of all such $a_{i}$ 's and

$$
\begin{aligned}
& \Phi\left(a_{i}\right)= \begin{cases}\text { a subset } I \subset \Phi ; & \begin{array}{l}
\text { The union of } I \text { and } a_{i} \text { is not completely } \\
\text { contained in any Delone } g \text { cell }
\end{array}\end{cases} \\
& \breve{\Phi}\left(a_{i}\right)=\left\{\begin{array}{ll}
a_{j} \in \Phi ; & \begin{array}{l}
\text { The union of } I \text { and } a_{j} \text { is not completely contained } \\
\text { in any Delone } g \text {-cell for any } I \text { of } \Phi\left(a_{i}\right)
\end{array}
\end{array}\right\}
\end{aligned}
$$

LEMMA 2.3. $\check{\Phi}\left(a_{i}\right)=a_{i}$ 
Proof. Assume $a_{j} \neq a_{i}, a_{j} \in \Phi$. Then there exists a Delone $g$-cell $\sigma$ containing the origin such that $\sigma \ni a_{j}, \sigma \notin a_{i}$ (obvious !) Denoting the set of vertices of $\sigma$ distinct from the origin by $I$, we have $I \in \Phi\left(a_{i}\right)$, and so $a_{j} \notin \Phi\left(a_{i}\right)$.

$$
\begin{aligned}
& \text { Obviously } \Phi\left(a_{i}\right)=\left\{I \subset \Phi ; x_{a_{i}} x_{I}=0\right\} \quad \text { where } \quad x_{I}=\prod_{a \in I} x_{a} \\
& \check{\Phi}\left(a_{i}\right)=\left\{a_{j} ; x_{a_{j}} x_{I}=0 \quad \text { for any } I \in \Phi\left(a_{i}\right)\right\}
\end{aligned}
$$

Any relation between $x_{a_{i}}$ 's in $\Gamma\left(X_{0} \mathcal{O}_{X_{0}}\right)$ is induced from proposition 2.3. (iii). So $\Omega_{X}^{1}$ is generated by $d x_{a_{i}}, a_{i} \in \Phi$, and $d x_{a_{i}}$ 's are subject to the relations induced by Proposition 2.3. (iii). We denote $P_{0} \cap U_{k}$ by $X_{k}$ $\left(k \in Z^{g}\right)$.

\section{LEMMA 2.4 .}

$$
\operatorname{Ext}^{0}\left(\Omega_{X_{0}}^{1}, \mathcal{O}_{X_{0}}\right)=\left\{\begin{array}{c}
\sum_{i} f_{a_{i}}(x) x_{a_{i}} \frac{\partial}{\partial x_{a_{i}}} ;\left(^{*}\right) \quad \sum_{i \in I} f_{a_{i}}=\sum_{i \in I} f_{a_{i}} \text { on } Z_{\sigma} \cap X_{0} \\
\text { for a pair }\left(I, I^{\prime}\right) \text { and } \sigma \text { satisfying (1) }
\end{array}\right\}
$$

where $x_{a_{i}}\left(\partial / \partial x_{a_{i}}\right)$ denotes an $\mathcal{O}_{X}$-homomorphism of $\Omega^{1}$ to $\mathcal{O}_{X}$ defined by $\left(x_{a_{i}}\left(\partial / \partial x_{a_{i}}\right)\right)\left(d x_{a_{j}}\right)=\delta_{i j} x_{a_{i}}\left(a_{j} \in \Phi\right), x_{a}=e\left(\frac{1}{2} a \tau_{0}(0)^{t} a\right) s^{a B^{t} a} e\left(a^{t} \zeta\right)$.

Proof. Assume $\omega$ is an element of $\operatorname{Ext}^{0}\left(\Omega_{X_{0}}^{1}, \mathcal{O}_{X_{0}}\right)$ defined by

$$
\omega\left(d x_{a_{i}}\right)=g_{a_{i}} \in \mathcal{O}_{X_{0}}, \quad a_{i} \in \Phi .
$$

For $I \in \Phi\left(a_{i}\right), x_{I} x_{a_{i}}=0$ hence $x_{a_{i}} d x_{I}+x_{I} d x_{a_{i}}=0$. Consequently

$$
0=\omega\left(x_{a_{i}} d x_{I}+x_{I} d x_{a_{i}}\right)=x_{a_{i}} \omega\left(d x_{I}\right)+x_{I} g_{a_{i}} .
$$

Therefore $x_{I}^{2} g_{a_{i}}=0$, hence we have $x_{I} g_{a_{i}}=0$ for $I \in \Phi\left(a_{i}\right)$. According to Lemma 2.2., we obtain $g_{a_{i}}=x_{a_{i}} f_{a_{i}}$ for some $f_{a_{i}} \in \mathcal{O}_{X_{0}}$. From the second relation (iii) of Proposition 2.3., it follows $\omega\left(d \prod_{i \in I} x_{a_{i}}\right)=\omega\left(d \prod_{i \in I^{\prime}} x_{a_{i}}\right)$, and since $\omega\left(d x_{I}\right)=\sum x_{I-a_{i}} \omega\left(d x_{a_{i}}\right)=x_{I}\left(\sum_{i \in I} f_{a_{i}}\right)$. We have $x_{I}\left(\sum_{i \in I} f_{a_{i}}\right.$ $\left.-\sum_{i \in I^{\prime}} f_{a_{i}}\right)=0$, which is equivalent to the equation $\sum_{i \in I} f_{a_{i}}=\sum_{i \in I^{\prime}} f_{a_{i}}$ on $Z_{\sigma} \cap X_{0}$. Conversely $\omega=\sum f_{a_{i}} x_{a_{i}}\left(\partial / \partial x_{a_{i}}\right)$ subject to $\left(^{*}\right)$ defines $a \mathcal{O}_{X_{0}}$ homomorphism of $\Omega_{X_{0}}^{1}$ to $\mathcal{O}_{X_{0}}$ in a canonical manner.

LEMMA 2.5. (i) $\quad 0 \rightarrow \mathscr{E} x t^{0}\left(\Omega_{X_{0}}^{1}, \mathcal{O}_{X_{0}}\right) \stackrel{\iota}{\longrightarrow} \mathcal{O}_{\tilde{X}}^{\sharp(\Phi)} \quad$ (exact)

(ii) Image of $\iota \cong \bigoplus_{0 \in \sigma_{g}} \mathcal{O}\left(X_{\sigma_{g}}\right)^{g}$ where $\iota(\omega)=\left(\pi_{\sigma}^{*} f_{a_{i}} a_{i} \in \sigma\right)$ with the notations in Lemma 2.3., $\pi_{\sigma}: X_{\sigma} \rightarrow \tilde{X}_{0}$ is an inclusion, $\tilde{X}_{0}=\cup_{0 \in \sigma} X_{\sigma}$ is the 
normalization of $X_{0}$.

Proof. At first we must show $\iota$ is well-defined. We take other $f_{a_{i}}$ 's such that

$$
\begin{gathered}
\omega=\sum f_{a_{i}}^{\prime} x_{a_{i}} \frac{\partial}{\partial x_{a_{i}}}=\sum f_{a_{i}} x_{a_{i}} \frac{\partial}{\partial x_{a_{i}}} . \\
0=\left(\sum_{i}\left(f_{a_{i}}^{\prime}-f_{a_{i}}\right) x_{a_{i}} \frac{\partial}{\partial x_{a_{i}}}\right)\left(d x_{a_{j}}\right)=\left(f_{a_{j}}^{\prime}-f_{a_{j}}\right) x_{a_{j}} .
\end{gathered}
$$

So we have $f_{a_{j}}^{\prime}=f_{a_{j}}$ on $X_{\sigma}$ if $a_{j} \in \sigma$, which shows $\iota$ is well-defined. It remains to show $\iota$ is injective. If $\iota(\omega)=0, \pi_{\sigma}^{*} f_{a_{i}}=0$ for any pair $a_{i} \sigma$. Therefore $\omega\left(d x_{a_{i}}\right)=f_{a_{i}} x_{a_{i}}=0$. We deduce the second assertion directly, noting that $\sum_{a_{i} \in I_{1}} f_{a_{i}}=\sum_{a_{i} \in I_{2}} f_{a_{i}}$, and $\sigma_{g}$ has exactly $g$ linearly independent vectors $x_{i}$.

Q.E.D.

LEMMA 2.6. The following sequence is exact,

$$
0 \longrightarrow \mathscr{E} x t^{0}\left(\Omega_{X_{0}}^{1}, \mathcal{O}_{X_{0}}\right) \stackrel{\iota}{\longrightarrow} \oplus \mathcal{O}\left(X_{\sigma_{g}}\right)^{g} \stackrel{\psi_{0}}{\longrightarrow} \oplus \mathcal{O}\left(X_{\sigma_{g-1}}\right)^{g-1}
$$

where $\mathcal{O}\left(X_{\sigma_{k}}\right)^{k}$ is the subsheaf $\mathcal{O}\left(X_{\sigma_{k}}\right)^{N\left(\sigma_{k}\right)}=\left\{\left(f_{a_{\sigma_{k}}}\right) ; \begin{array}{l}a \in \sigma_{k} \cap \Phi \\ f_{a, \sigma_{k}} \in \mathcal{O}\left(X_{\sigma_{k}}\right)\end{array}\right\}\left(N\left(\sigma_{k}\right)=\right.$ $\left.\#\left(\sigma_{k} \cap \Phi\right)\right)$ defined by

$$
\sum_{i \in I_{1}} f_{a_{i}, \sigma}=\sum_{i \in I_{2}} f_{a_{i, \sigma}} \quad \text { if } \quad \sum_{i \in I_{1}} a_{i}=\sum_{i \in I_{2}} a_{i}
$$

and $\psi_{0}$ is defined by

$$
\psi_{0}\left(\left(f_{a, \sigma}\right)_{a \in \sigma}\right)=\left(f_{a_{\tau}}\right), \quad f_{a_{\tau}}=\sum_{\tau<\sigma}[\sigma: \tau] f_{a_{\sigma}} .
$$

Proof. According to Lemma 2.4., it is shown that $\psi_{0}$ is exact (i.e., injective) and the image of $\psi_{0}$ is $\left\{\left(\pi_{\sigma}^{*} f_{a_{i}}\right) a_{i} \in \sigma, f_{a_{i}} \in \mathcal{O}\left(X_{0}\right)\right\}$, which is nothing other than $\operatorname{Ker} \psi_{1}$ because $[\sigma: \tau]=-\left[\sigma^{\prime}: \tau\right]$ for any $(g-1)$ cell $\tau$ and adjacent pair $\sigma, \sigma^{\prime}$ such that $\sigma \cap \sigma^{\prime}=\tau$.

Any $X_{k}$ is isomorphic, so there is the same kind of an exact sequence on $X_{k}$. Patching them together we obtain a global exact sequence as follows,

Proposition 2.5. The following sequence is exact;

$$
0 \longrightarrow \mathscr{E} x t^{0}\left(\Omega_{X}^{1}, \mathcal{O}_{X}\right) \stackrel{\iota}{\longrightarrow} \underset{\sigma_{g} \bmod \Gamma}{\oplus} \mathcal{O}\left(X_{\sigma_{g}}\right)^{g} \stackrel{\psi_{0}}{\longrightarrow} \underset{\sigma_{g-1} \bmod \Gamma}{\bigoplus_{1}} \mathcal{O}\left(X_{\sigma_{g-1}}\right)^{g-1}
$$

Proof. We remark that $X_{0} \cap X_{k} \neq \phi(k \neq 0)$ if and only if $k=a_{i} \in \Phi$. 
On $X_{0} \cap X_{k}$ we shall investigate the relation between two homomorphism $\left.\psi_{0}\right|_{X_{0}}$ and $\left.\psi_{0}\right|_{X_{k}}$.

Let $k$ be an element of $\Phi$, say $a_{1}$. Let

$$
\Gamma\left(X_{0}\right)=C\left[x_{a_{i}}, a_{i} \in \Phi\right], \quad x_{a_{i}}=e\left(\frac{1}{2} a_{i} \tau_{0}^{t} a_{i}\right) s^{a_{i} B^{t} a_{i}} e\left(a_{i}{ }^{t} \zeta\right)
$$

and

$$
\Gamma\left(X_{a_{1}}\right)=C\left[x_{a_{i}}^{\prime}, a_{i} \in \Phi\right], \quad x_{a_{i}}^{\prime}=e\left(\frac{1}{2} a_{i} \tau_{0}^{t} a_{i}+a_{i} \tau_{0}^{t} a_{1}\right) s^{2 a_{i} B^{t} a_{1}+a_{i} B^{t} a_{i}} e\left(a_{i}{ }^{t} \zeta\right) .
$$

$X_{0}$ and $X_{a_{1}}$ are glued along $X_{0} \cap X_{a_{1}}$ by the relation

$$
\begin{aligned}
x_{a_{j}}^{\prime} & =0 & & \text { if } a_{j}+a_{1} \notin \Phi \\
x_{a_{j}} & =0 & & \text { if } a_{j}-a_{1} \notin \Phi \\
x_{-a_{1}}^{\prime} & =x_{a_{1}}^{-1} & & \\
x_{a_{j}-a_{1}}^{\prime} & =x_{a_{1}}^{-1} x_{a_{j}} & & \text { if } a_{j}-a_{1} \in \Phi .
\end{aligned}
$$

Hence we have

$$
\begin{aligned}
d x_{-a_{1}}^{\prime} & =-x_{a_{1}}^{-2} d x_{a_{1}} \\
d x_{a_{j}-a_{1}}^{\prime} & =-x_{a_{1}}^{-2} d x_{a_{j}}+x_{a_{1}}^{-1} x_{a_{j}} d x_{a_{j}} \quad\left(a_{j}-a_{1} \in \Phi\right) .
\end{aligned}
$$

Let $\omega$ be an element of $\operatorname{Ext}^{0}\left(\Omega^{1} X_{0} \cap X_{k}, 0_{X_{0} \cap X_{k}}\right)$ given by

$$
\omega=\sum_{a_{i} \in \Phi} f_{a_{i}} x_{a_{i}} \frac{\partial}{\partial x_{a_{i}}}=\sum_{a_{i} \in \Phi} f_{a_{i}}^{\prime} x_{a_{i}}^{\prime} \frac{\partial}{\partial x_{a_{i}}^{\prime}} .
$$

Then computing $\omega\left(d x_{-a_{1}}^{\prime}\right), \omega\left(d x_{a_{j-a_{1}}}^{\prime}\right)$ we obtain,

$$
f_{-a_{1}}^{\prime}=-f_{a_{1}}, \quad x_{a_{j}}\left(f_{a_{j}-a_{1}}^{\prime}-f_{a_{j}}+f_{a_{1}}\right)=0 \quad\left(a_{j}-a_{1} \in \Phi\right),
$$

hence

$$
\pi_{\sigma}^{*} f_{-a_{1}}^{\prime}=-\pi_{\sigma}^{*} f_{a_{1}}, \quad \pi_{\sigma}^{*} f_{a_{j}-a_{1}}^{\prime}=\pi_{\sigma}^{*} f_{a_{j}}-\pi_{\sigma}^{*} f_{a_{1}} \quad\left(a_{j} \in \sigma, a_{j}-a_{1} \in \Phi\right) .
$$

If we fix a $Z$-basis $e_{j}(j=1, \cdots, g)$ of $Z^{g}$ and define

$$
F_{a_{j^{\sigma}}}=\pi_{\sigma}^{*} f_{a_{j}}, F_{a_{\sigma}}=\sum_{i} n_{i} \pi_{\sigma}^{*} f_{a_{i}} \text { for } a=\sum_{i} n_{i} a_{i}\left(n_{i} \in Z\right), F_{a}^{\prime} \text { likewise, }
$$
then the above isomorphism on $X_{0} \cap X_{k}$ reduces to the trivial identities $F_{e_{i \sigma}}=F_{e_{i \sigma} \sigma}^{\prime}$. On the other hand the exact sequence thus obtained on (Proj $R)_{0}$ can be descended to $X$ because of the invariance under the action of the lattice $\Gamma$.

Q.E.D.

THEOREM 2.1. Under the assumption $(A)$ and $(B), \operatorname{dim} \operatorname{Ext}^{0}\left(\Omega_{X}, \mathcal{O}_{X}\right)$ $=g$ if $\lambda=1$.

Remark. We prove here the above theorem in case that $B$ is positive. 
But as easily shown, we infer from the fact of $\operatorname{dim} \operatorname{Ext}^{0}\left(\Omega_{A}^{1}, \mathcal{O}_{A}\right)=g$ for an nonsingular aberian variety that even in the general case our theorem under the same assumption is true. On the other hand our theorm is not true if $\lambda \neq 1$. For example, $\left.\operatorname{dim} \operatorname{Ext}^{0}\left(\Omega_{X}^{1}, \mathcal{O}\right)\right)=n$ for a chain of lines. $(\lambda=n, g=1)$.

Proof of the Theorem. By virtue of proposition 2.5. we have only to compute the kernel of $\psi_{0}: \oplus H^{0}\left(\mathcal{O}\left(X_{\sigma_{g}}\right)\right)^{g} \rightarrow \oplus H^{0}\left(\mathcal{O}\left(X_{\sigma_{g-1}}\right)\right)^{g-1}$. A section $\left(C_{a, \sigma} a \in \sigma_{g} \cap \Phi\right)$ of $\oplus H^{0}\left(\mathcal{O}\left(X_{\sigma_{g}}\right)\right)^{g}$ is mapped to $\left(C_{a \sigma}-C_{a_{\sigma}}\right)$ for adjacent pair $\sigma$ and $\sigma^{\prime}$, i.e. $\sigma \cap \sigma^{\prime}$ is a $(g-1)$ cell. $\left(C_{a \sigma}\right)$ is contained in $\operatorname{Ker} \psi_{1}$ if and only if $C_{a \sigma}$ is independent of $\sigma$, i.e. $C_{a \sigma}=C_{a}$. Moreover if $a_{i} \in \sigma$, $\sum_{i \in I} a_{i}=0 \bmod \Gamma$, then $\sum_{i \in I} C_{a_{i}}=0$. Since $\lambda=1$, there are at most $g$ linearly independent solution by the assumption $(B) . \quad\left(C^{*}\right)^{g}$ acts effectively on $X$, so $\left.\operatorname{dim} \operatorname{Ext}^{0}\left(\Omega_{X}^{1}, \mathcal{O}_{X}\right)\right)=g$.

Q.E.D. More precisely we obtain

THEOREM 2.2. Let $\Sigma$ be a $D-V$ cone corresponding to a $S Q A V X$ of level 1 , and $\Sigma \subset \mathfrak{Y}_{g}^{+}$. Then under the assumptions $(A)$ and $(B)$ we have $\operatorname{Aut}^{1} X=$ Aut $\Sigma$ where $\operatorname{Aut}^{1} X=\operatorname{Aut} X / \operatorname{Aut}^{0} X$, Aut $\Sigma=\{u \in G L(g Z)$; $\left.u \Sigma^{t} u=\Sigma\right\}$.

Proof. By the assumption that $\Sigma \subset \mathfrak{Y}_{g}^{+}$, any irreducible component $X_{\sigma}$ is a compactification of an algebraic torus $\left(C^{*}\right)^{g}$. Fix a Delone $g$ cell $\sigma_{0}$ and an embedding of $\left(C^{*}\right)^{g}$ into $X_{\sigma_{0}}$. Then assumption $(B)$ implies that for any $g$ cell $\sigma$ in a canonical way an embedding of $\left(C^{*}\right)^{g}$ into $X_{\sigma}$ is uniquely determined. We fix a $Z$-basis of $Z^{g}$. An automorphism $f$ of $X$ induces a polyhedral automorphism $\bar{f}$ of the Delone decomposition associated with $\Sigma$. If two $g$ cells $\sigma_{1}$ and $\sigma_{2}$ are adjacent, then $\bar{f}\left(\sigma_{1}\right)$ and $\bar{f}\left(\sigma_{2}\right)$ are adjacent. $\bar{f}$ on $\sigma_{i}$ is represented by a matrix $u_{\sigma_{i}}$ of $G L(g Z)$ w.r.t. the above basis of $Z^{g}$ and in view of the assumption $(B)$, we have $u_{\sigma_{1}}=u_{\sigma_{2}}$. By induction on the length of a chain of Delone $g$ cells connecting $\sigma$ and $\sigma_{0}$, we have $u_{\sigma}=u_{\sigma_{0}}$, namely, $\bar{f}$ is induced from a linear transformation $u=u_{\sigma_{0}}$ of $\boldsymbol{R}^{g}$, and $u \in$ Aut $\Sigma$. Obviously $u$ induces an automorphism of $X$, so we consider an automorphism $f^{\prime}=f \circ u^{-1}$ of $X$. $f^{\prime}$ transforms $X_{\sigma}$ into itself, moreover, $f^{\prime}$ is a translation by a constant vector $a_{\sigma}\left(\in\left(C^{*}\right)^{g}\right)$ on $X_{\sigma}$. $f^{\prime}$ is an element of Aut $^{0} X=$ the connected component of identity of Aut $X$. Hence by the theorem 2.1., we obtain $a_{\sigma}=a$ independent of $\sigma$ if $\lambda=1$.

Q.E.D. 
Remark. Notice that in the above theorems we mean by an automorphism of $X$ simply that as an algebraic variety, not as a SQAV. Therefore an element $u$ of Aut $X$ may not preserve a section $X$ over $\boldsymbol{C}$.

\section{§. Voronoi compactification and Namikawa's polarized family of SQAV over it.}

(3.1) As stated in the introduction Namikawa gave a precise construction of the Voronoi compactification in [18] by using the theory of troidal embeddings [11]. The Voronoi compactification $\widetilde{\Im}_{g}^{*}(\lambda)$ has been already known as the Igusa monoidal transform of Satake compactification of Siegel space if $g \leqq 3$. It is deeply related with our stable quasi abelian varieties. In fact, Namikawa succeeded in constructing a global polarized family of stable quasi abelian varieties over it. We shall introduce an outline of his results in (3.1) (3.2). In (3.3) we shall investigate the structure of the Voronoi compactification $\widetilde{\Xi}_{g}^{*}(\lambda)$ along the boundary, in particular, the fibering over $\widetilde{\varsigma}_{g-1}^{*}(\lambda)$ of an irreducible component of the boundary of codimension one. We notice that a general fiber of this fibering is a polarized abelian variety with level $\lambda$ structure. Therefore it seems natural to expect the fibers of this fibering are also our stable quasi abelian varieties. However this is not the case. We find that a reducible variety distinct from our stabel quasi abelian variety appears as fiber. But this does not imply that our definition is improper, on which we shall give a comment in $\S 7$. Set

$$
\begin{aligned}
B(\lambda) & =\left\{M=\left(\begin{array}{cc}
1 & B \\
0 & 1
\end{array}\right) ; B \in M_{g}(Z),{ }^{t} B=B, B \equiv 0 \bmod \lambda\right\}, \\
e(x) & =\exp (2 \pi \sqrt{-1} x) \\
\Gamma_{g} & =\operatorname{Sp}(g Z), \quad \Gamma_{g}(\lambda)=\operatorname{Sp}(g Z)(\lambda)=\{M \in \operatorname{Sp}(g Z) ; M \equiv 1 \bmod \lambda\} \\
T_{g} & =\left\{W=\left(w_{i j}\right) ;{ }^{t} W=W, w_{i j} \neq 0\right\} \cong\left(C^{*}\right)^{g(g+1) / 2} \\
T_{g}^{0} & =\left\{W \in T_{g} ; \log W=\left(\log \left|w_{i j}\right|\right) \text { is negative definite }\right\} \\
\mathfrak{Y}_{g}^{+}(r) & =\left\{y \in \mathfrak{Y}_{g}^{+} ; k y^{t} k>r \text { for any non zero integral vector } k\right\} .
\end{aligned}
$$

We fix $\lambda$ once for all. The mapping $e(\lambda)$ from $\widetilde{S}_{g}$ to $T_{g}$ defined by $\left.e(\lambda)(\tau)=\left(e\left((1 / \lambda) \tau_{i j}\right)\right)\right)$ induces an isomorphism between $\mathfrak{S}_{g} / B(\lambda)$ and $T_{g}$. We identify them through this isomorphism.

Let $\Sigma$ be a $\mathrm{D}-\mathrm{V}$ cone in $\overline{\mathfrak{Y}}_{g}^{+}$. Then by an appropriate transformation $u$ of $G L(g Z), \Sigma$ is transformed into a D-V cone $\left\{\left(\begin{array}{cc}0 & 0 \\ 0 & y^{\prime \prime}\end{array}\right) ; y^{\prime \prime} \in \Sigma^{\prime \prime}\right\}$ 
for a $\mathrm{D}-\mathrm{V}$ cone $\Sigma^{\prime \prime}$ in $\mathfrak{Y}_{g^{\prime \prime}}^{+}$. (Notice that not only $\Sigma^{\prime \prime} \subset \mathfrak{Y}_{g^{\prime \prime}}^{+}$). Therefore we assume

$$
\Sigma=\left\{\left(\begin{array}{cc}
0 & 0 \\
0 & y^{\prime \prime}
\end{array}\right) ; y^{\prime \prime} \in \Sigma^{\prime \prime}\right\}, \quad \Sigma^{\prime \prime} \subset \mathfrak{V}_{g^{\prime \prime}}^{+}
$$

For brevity we write

$$
\tau=\left(\begin{array}{cc}
t_{11} & t_{12} \\
& t_{1}
\end{array}\right)
$$

or $\left(t_{1}, t_{12}, t_{2}\right)$ instead of $\tau_{i j}$, where $t_{1} \in \widetilde{S}_{g}, t_{2} \in \widetilde{S}_{g^{\prime \prime}}, t_{12} \in M_{g^{\prime} g^{\prime \prime}}(C), g=g^{\prime}+g^{\prime \prime}$.

Then we define $\mathscr{Z}_{\Sigma^{\prime \prime}}$ to be a troidal embedding of $T_{g^{\prime \prime}}$ associated with $\Sigma^{\prime \prime}$ i.e.

$$
\mathscr{Z}_{\Sigma^{\prime \prime}}=\operatorname{Spec} C\left[e\left(\frac{1}{\lambda} \operatorname{tr}(a \tau)\right) ; a \in\left(\Sigma^{\prime \prime}\right)_{Z}^{\vee}\right]
$$

and

$$
\mathscr{X}_{\Sigma}=\operatorname{Spec} C\left[e\left(\frac{1}{\lambda} \operatorname{tr}(a \tau)\right) ; a \in(\Sigma)_{Z}^{\vee}\right] \cong T_{g^{\prime}} \times\left(C^{*}\right)^{g^{\prime} \theta^{\prime \prime}} \times \mathscr{Z}_{\Sigma^{\prime \prime}}
$$

Let

$$
\begin{aligned}
T_{g g^{\prime \prime}}^{0}(r) & =\left\{W=\left(\begin{array}{rr}
e\left(t_{1}\right) & e\left(t_{12}\right) \\
& e\left(t_{2}\right)
\end{array}\right) ; \operatorname{Im} t_{2}-L_{t_{1}}\left(t_{12}\right) \in \mathfrak{Y}_{g^{\prime \prime}}^{+}(r)\right\} \\
T_{g g^{\prime \prime}}^{0} & =T_{g g^{\prime \prime}}^{0}(0) \quad \text { where } L_{t_{1}}\left(t_{12}\right)={ }^{t} \operatorname{Im}\left(t_{12}\right)\left(\operatorname{Im} t_{1}\right)^{-1} \operatorname{Im}\left(t_{12}\right) .
\end{aligned}
$$

DEFINITION 3.1. $S_{g^{\prime \prime}}\left(V, \Sigma^{\prime \prime}, r\right)=$ the interior of the closure of $T_{g g^{\prime \prime}}^{0}(r)$ in $\left(V \times\left(C^{*}\right)^{g^{\prime} g^{\prime \prime}} \times \mathscr{Z}_{\Sigma^{\prime \prime}}\right)$ where $V$ is an open set in

$$
T_{g^{\prime}}^{0}, \Sigma=\left\{\left(\begin{array}{cc}
0 & 0 \\
0 & y^{\prime \prime}
\end{array}\right) ; y^{\prime \prime} \in \Sigma^{\prime \prime}\right\}
$$

If $\Sigma_{1}^{\prime \prime} \prec \Sigma_{2}^{\prime \prime}$, then $S_{g^{\prime \prime}}\left(V, \Sigma_{1}^{\prime \prime}, r\right)$ can be canonically embedded into $S_{g^{\prime \prime}}\left(V, \Sigma_{2}^{\prime \prime}, r\right)$. If $V_{1} \subset V_{2}$, then $S_{g^{\prime \prime}}\left(V_{1}, \Sigma_{1}^{\prime \prime}, r\right) \subset S\left(V_{2}, \Sigma_{2}^{\prime \prime}, r\right)$. Gluing $S(V$, $\left.\Sigma^{\prime \prime}, r\right)$ together, we set $\mathscr{X}_{\Sigma}^{0}=\bigcup_{V} S\left(V, \Sigma^{\prime \prime}, r\right), S_{g^{\prime \prime}}(V, r)=\bigcup_{\Sigma^{\prime \prime}} S_{g^{\prime \prime}}\left(V, \Sigma^{\prime \prime}, r\right)$. For a general $\Sigma$, we define $S(V, \Sigma, r)$ to be the pull back by a tranformation of $G L(g Z)$ of $S\left(V, \Sigma^{\prime \prime}, r\right)$. We set $\mathscr{X}^{0}=T_{g}^{0} \bigcup_{\substack{r, T \in G L(g Z) \\ \Sigma^{\prime \prime}, V}} T^{-1} S\left(V, \Sigma^{\prime \prime}, r\right)$. and $G L(g Z)$ operates on $\mathscr{X}^{0}$.

For a point $t_{1}^{0}$ in $T_{g}$, the stabilizer subgroup $H\left(t_{1}^{0}\right)$ is finite. Hence we can choose a sufficiently small neighborhood $V$ of $t_{1}^{0}$ such that $M_{1} V \cap V$ $\neq \phi$ if and only if $M_{1} \in H\left(t_{1}^{0}\right)$, and $M_{1} V=V$ for any $M_{1} \in H\left(t_{1}^{0}\right)$. Then we put, 


$$
\Gamma_{g g^{\prime \prime}}\left(t_{1}^{0}\right)(\lambda)=\left\{\left(\begin{array}{cccc}
a_{11} & 0 & b_{11} & b_{12} \\
a_{21} & a_{22} & b_{21} & b_{22} \\
c_{11} & 0 & d_{11} & d_{12} \\
0 & 0 & 0 & d_{22}
\end{array}\right) \in S p(g Z) ;\left(\begin{array}{ll}
a_{11} & b_{11} \\
c_{11} & d_{11}
\end{array}\right) \in H\left(t_{1}^{0}\right)\right\}
$$

Obviously $B_{g}(\lambda)$ is a normal subgroup of $\Gamma_{g g^{\prime \prime}}\left(t_{1}^{0}\right)(\lambda)$, so we set $\bar{\Gamma}_{g g^{\prime \prime}}\left(t_{1}^{0}\right)(\lambda)=\Gamma_{g g^{\prime \prime}}\left(t_{1}^{0}\right)(\lambda) / B_{g}(\lambda)$. If $\lambda \geqq 3$, then $H\left(t_{1}^{0}\right)=1$, hence we have $a_{11}$ $=d_{11}=1, b_{11}=c_{11}=0$ for an element $M$ of $\Gamma_{g g^{\prime \prime}}\left(t_{1}^{0}\right)(\lambda)$. Assume $\lambda \geqq 3$, $M \in \bar{\Gamma}_{g g^{\prime \prime}}\left(t_{1}^{0}\right)(\lambda), M \cap S_{g^{\prime \prime}}\left(V, \Sigma^{\prime \prime}, r\right) \cap S_{g^{\prime \prime}}\left(V, \Sigma^{\prime \prime}, r\right) \neq \phi$ for a $\mathrm{D}-\mathrm{V}$ cone $\Sigma^{\prime \prime}\left(\subset \mathfrak{Y}_{g^{\prime \prime}}^{+}\right)$ and a sufficiently large $r>0$. Let $\left(t_{1}^{\prime} t_{12}^{\prime} t_{2}^{\prime}\right)=M \cdot\left(t_{1} t_{12} t_{2}\right)$. Then we have

$$
\left(\operatorname{Im} t_{2}^{\prime}-\operatorname{Re}\left(L_{t_{1}}\left(t_{12}^{\prime}\right)\right)=a_{22}\left(\operatorname{Im} t_{2}-\operatorname{Re}\left(L_{t_{1}}\left(t_{12}\right)\right)^{t} a_{22} .\right.\right.
$$

We notice $a_{22} \in G L\left(g^{\prime \prime} Z\right)$. Moreover a general point $\left(t_{1} t_{12} t_{2}\right)$ of $S_{g^{\prime \prime}}\left(V, \Sigma^{\prime \prime}, r\right)$ satisfies

$$
\operatorname{Im} t_{2}-\operatorname{Re}\left(L_{t_{1}}\left(t_{12}\right)\right) \in \Sigma^{\prime \prime} \cap \mathfrak{Y}_{g^{\prime \prime}}^{+}(r) .
$$

Hence $a_{22} \in$ Aut $\Sigma^{\prime \prime}$. In view of Proposition 1.2., Aut $\Sigma^{\prime \prime}$ is finite, so that $a_{22}=1$ if $\lambda \geqq 3$. Hence $\Gamma_{g g^{\prime \prime}}\left(t_{1}^{0}\right)(\lambda)$ consists of elements such that $a_{11}=$ $a_{22}=d_{11}=d_{22}=1, b_{11}=c_{11}=0 . \quad(\lambda \geqq 3)$. Then it is easy to check $\Gamma_{g g^{\prime \prime}}\left(t_{1}^{0}\right)(\lambda)$ operates properly discontinuously and freely. Therefore the quotient of $S(V, r)$ by $\Gamma_{g g^{\prime \prime}}\left(t_{1}^{0}\right)(\lambda)$ exists and becomes a normal complex space. Notice that if $S(V, r)$ is nonsingular, so is the quotient. If $\lambda=1$ or 2 , then $\Gamma_{g g^{\prime \prime}}\left(t_{1}^{0}\right)(k \lambda)(k \lambda \geqq 3)$ is of finite index in $\Gamma_{g g^{\prime \prime}}\left(t_{1}^{0}\right)(\lambda)$, so in this case a quotient space $S(V, r)$ by $\Gamma_{g g^{\prime \prime}}\left(t_{1}^{0}\right)(\lambda)$ exists and becomes a normal complex space.

Remark. The author does not know whether the following statement is true, for $g^{\prime \prime}<g$

: For a point $t_{1}^{0}$ in $T_{g}^{0}$ a sufficiently small neighborhood $V$ of $t_{1}^{0}$ and a sufficiently large $r>0, S p(g Z)$-equivalence on $S_{g^{\prime \prime}}(V, r) \cap T_{g}^{0}$ reduces to $\Gamma_{g g^{\prime \prime}}\left(t_{1}^{0}\right)$-equivalence.

THEOREM (Mumford [14], Namikawa [18]). The quotient space $\mathfrak{S}_{g}^{*}(\lambda)$ $=\mathscr{X}^{0} / \Gamma_{g}(\lambda)$ exists and is a normal compact complex space containing $\mathfrak{S}_{g} / \Gamma_{g}(\lambda)$ as a Zariski dense open subset.

(3.2) Next we shall introduce Namikawa's construction of a polarized family of SQAV over the Voronoi compactification. As stated in $\S 1$, his method is an application of troidal embeddings.

DEFINITION 3.2. $\quad V_{\Sigma \sigma}$ is a mixed $\mathrm{D}-\mathrm{V}$ cone associated with $\Sigma$ and $\sigma$ 
where is a $\mathrm{D}-\mathrm{V}$ cone and $\sigma=\left\langle a_{i}, i \in I\right\rangle=\left\{\sum_{\nu=0}^{r} \lambda_{\nu} a_{i_{\nu}} ; 0 \leqq \lambda_{\nu} \leqq 1, \sum_{\nu=0}^{r} \lambda_{\nu}=1\right.$, $\left.i_{\nu} \in I\right\}$ is a Delone cell associated with $\Sigma$, if

$$
\begin{aligned}
& V_{\sigma \Sigma}=\left\{(y, x) \in \bar{\Sigma} \times \boldsymbol{R}^{g} ; m y^{t}\left(m+2 a_{i}\right)+m^{t} x \geqq 0\right. \\
& \left.\qquad \text { for any } m \in \boldsymbol{Z}^{g}, \text { and } i \in I\right\}
\end{aligned}
$$

Then $\left\{V_{\Sigma \sigma}\right\}$ forms a polyhedral decomposition of $\overline{\mathfrak{Y}}_{g}^{+} \times R^{g}$

Definition 3.3. $\left\{V_{\Sigma \sigma}\right\}$ is called a mixed V-D decomposition of $\overline{\mathfrak{Y}}_{g}^{+} \times \boldsymbol{R}^{g}$.

Proposition 3.1 (Namikawa [18]).

$$
V_{\Sigma \sigma} \cap\left(y_{0} \times R^{g}\right)=\Delta(\sigma) \text { with respect to } y_{0} \text {. }
$$

DEFINITION 3.4. $\mathscr{P}_{\Sigma \sigma}=$ the troidal embedding of $T_{g} \times\left(C^{*}\right)^{g}$ associated with $V_{\Sigma_{\sigma}}=\operatorname{Spec} R_{\Sigma \sigma}$ where

$$
R_{\Sigma \sigma}=C\left[e\left(\frac{1}{2 \lambda} \operatorname{tr}(a \tau)+m^{t} \zeta\right),(a, m) \in\left(\check{V}_{\Sigma \sigma}\right)_{z}\right] .
$$

DEFINITION 3.5. $\quad \mathscr{P}_{\Sigma \sigma}^{0}=\mathscr{P}_{\Sigma \sigma} \times_{\mathscr{x}_{\Sigma}} \mathscr{X}_{\Sigma}^{0}, \mathscr{P}_{\Sigma}^{0}=\bigcup_{\sigma} \mathscr{P}_{\Sigma \sigma}^{0}$ and $\mathscr{P}^{0}=\bigcup_{\Sigma} \mathscr{P}_{\Sigma}^{0}$, where $\mathscr{X}_{\Sigma}=\operatorname{Spec} C\left[e((1 / 2 \lambda) \operatorname{tr}(\alpha \tau)), a \in(\Sigma)_{Z}^{\vee}\right]$. If $\Sigma_{1}$ is a face of $\Sigma_{2}$, then $\mathscr{P}_{\Sigma_{1}}^{0} \subset \mathscr{P}_{\Sigma_{2}}^{0}$, canonically. So gluing them, we obtain $\mathscr{P}^{0}=\bigcup_{\Sigma} \mathscr{P}_{\Sigma}^{0}$. We define an invertible sheaf $\mathscr{L}^{0}$ on $\mathscr{P}^{0}$ by,

$$
\mathscr{L}^{0}=U \mathscr{L}_{\Sigma k}^{0}, \quad \mathscr{L}_{\Sigma k}^{0}=e\left(\frac{1}{2 \lambda} \operatorname{tr}\left({ }^{t} k k \tau\right)+k^{t} \zeta\right) \mathcal{O}_{g^{0} 0 k} .
$$

Then $Z^{g}$ acts on both $\mathscr{P}^{0}$ and $\mathscr{L}^{0}$ freely and properly discontinuously via $S_{n}\left(n \in Z^{g}\right)$ as follows; $\mathscr{P}_{\Sigma}^{0}$ is covered with $\mathscr{P}_{\Sigma k}^{0}\left(k \in Z^{g}\right)$. The transformation $S_{n}$ is defined by,

$$
\begin{aligned}
& S_{n}^{*}: R_{\Sigma k+n} \rightarrow R_{\Sigma k} \\
& \left.S_{n}^{*}\left(e\left(\frac{1}{2 \lambda} \operatorname{tr}(a \tau)+m^{t} \zeta\right)\right)\right)=e\left(\frac{1}{2 \lambda} \operatorname{tr}\left(\left(2^{t} n m+a\right) \tau\right)+m^{t} \zeta\right) \\
& S_{n}: \mathscr{L}_{\Sigma k}^{0} \rightarrow \mathscr{L}_{\Sigma k+n}^{0} \\
& S_{n}(c)=e\left(\frac{1}{2 \lambda} \operatorname{tr}\left({ }^{t} n n \tau+2^{t} n k \tau\right)+n^{t} \zeta\right) c
\end{aligned}
$$

Namikawa has proved the action of $\operatorname{Sp}(g Z)(2 \lambda)$ on $\mathbb{S}_{g}$ can be lifted to $\mathscr{A}^{0}=\mathscr{P}^{0} / \boldsymbol{Z}^{g}, \theta^{0}=\mathscr{L}^{0} / \boldsymbol{Z}^{g}$, also $\theta^{0}$ is relatively ample on $\mathscr{A}^{0}$.

THEOREM 3.2 (Namikawa). There exists a principally polarized 
family of SQAV's of level $\lambda$ over the Voronoi compactification $\widetilde{\subseteq}_{g}^{*}(2 \lambda)$ $=\mathscr{X}^{0} / \Gamma_{g}(2 \lambda)$.

There's another construction of a family of SQAV's over the Voronoi compactification by use of $\operatorname{Proj}(*)$ as in $\S 2$. By the same argument as before if $g \leqq 4$, they coincide with each other.

(3.3) Next we shall investigate an irreducible component of the complement of $\widetilde{S}_{g} / \Gamma_{g}(\lambda)$ in $\mathscr{X}^{0} / \Gamma_{g}(\lambda)$, which we call an irreducible component of the boundary for brevity. At least if $g$ is not greater than 3, $\mathscr{X}_{g}^{0} / \Gamma(\lambda)$ is non singular $(\lambda \geqq 3)$, and any irreducible component of the boundary is of codimension one.

Although the results are already known in [8], we recall them from the view point of troidal embeddings for later use.

By the general theory of troidal embeddings, we know that there is a one to one correspondence between irreducible components of the boundary and one-dimensional D-V cones. So with loss of generality we may assume a corresponding $\mathrm{D}-\mathrm{V}$ cone is $\tau_{0}=\left\{\left(\begin{array}{ll}0 & 0 \\ 0 & y\end{array}\right), \begin{array}{c}y>0 \\ (y \in R)\end{array}\right\}$, and we denote by $X_{\tau_{0}}$ an irreducible component of the boundary associated with $\tau_{0}$. Since $\widetilde{\Xi}_{g}^{*}(\lambda)$ is covered with open sets $\mathscr{X}_{\Sigma}^{0}$, ( $\Sigma$ : open $\mathrm{D}-\mathrm{V}$ cones $)$, we suffices to consider only $\mathscr{X}_{\Sigma}^{0}$ for open $\mathrm{D}-\mathrm{V}$ cones in order to investigate the structure of $X_{\tau_{0}}$. We notice that $X_{\tau_{0}}$ is contained in $\mathscr{X}_{\Sigma}^{0}$ if and only if $\tau_{0}$ is a face of $\Sigma$. So we have only to pick up all open $\mathrm{D}-\mathrm{V}$ cones containg $\tau_{0}$, however it can be readily carried out by using a well known classification of open $\mathrm{D}-\mathrm{V}$ cones up to $G L(g Z)$ conjugate in case of $g=$ 2,3 .

At first we consider the case where $g=2$. All open $\mathrm{D}-\mathrm{V}$ cones which have $\tau_{0}$ as a face are listed as follows,

$$
\begin{gathered}
D_{n}=\left\{\left(\begin{array}{cc}
y_{11} & y_{12} \\
y_{12} & y_{22}
\end{array}\right) \in M_{2}(\boldsymbol{R}) ; \begin{array}{c}
n y_{11}-y_{12}>0,-(n-1) y_{11}+y_{12}>0 \\
n(n-1) y_{11}-(2 n-1) y_{12}+y_{22}>0
\end{array}\right\} \quad(n \in Z) \\
X_{\tau_{0}} \cap \mathscr{X}_{D_{n}}^{0}=\left\{\left(s_{11}^{n} s_{12}^{-1}, s_{11}^{-n+1} s_{12}\right) ;\left|\left(s_{11}^{n} s_{12}^{-1}\right)\left(s_{11}^{-n+1} s_{12}\right)\right|<1\right\}
\end{gathered}
$$

where $s_{i j}=\exp \left(2 \pi i \tau_{i j} / \lambda\right)$

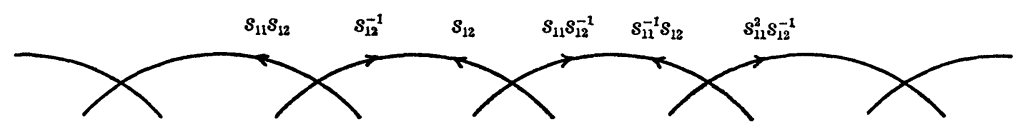


The action of $S p(2 Z)(\lambda)$ on $X_{\tau_{0}}$ reduces to that of $G L(2 Z)(\lambda)$, hence in particular if $\lambda \geqq 3$, reduces to the following isomorphisms,

$$
\begin{gathered}
\mathscr{X}_{\tau_{0}} \cap \mathscr{X}_{D_{n}}^{0} \cong \mathscr{X}_{\tau^{0}} \cap \mathscr{X}_{D_{n^{\prime}}}^{0} \quad\left(n \equiv n^{\prime} \bmod \lambda\right) \\
\left(s_{11}^{n} s_{12}^{-1}, s_{11}^{-n+1} s_{12}\right) \rightarrow\left(s_{11}^{n^{\prime}} s_{12}^{-1}, s_{11}^{-n^{\prime}+1} s_{12}\right)
\end{gathered}
$$

$X_{\tau_{0}}$ has an elliptic fibering over $\mathfrak{S}_{1}^{*}(\lambda)$ defined by, $t=\left(s_{11}^{n} s_{12}^{-1}\right)\left(s_{11}^{-n+1} s_{12}\right)$ $\left(t=\exp (2 \pi i z / \lambda), z \in \mathfrak{S}_{1}\right)$. The fiber at $t=t_{0} \neq 0$ is an elliptic curve with period $\left(1, \lambda \log t_{0} / \sqrt{-1}\right)$, and the fiber at $t=0$ is a chain of $\lambda$-lines. Moreover $X_{\tau_{0}}$ carries the level $\lambda$ structure defined by, $s_{11}^{n} s_{12}^{-1}=$ one of $\lambda$-th root of unity.

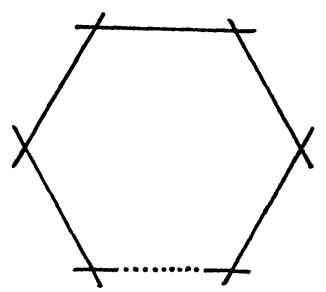

Next we consider the case where $g=3$. Then all the open $\mathrm{D}-\mathrm{V}$ cones dominating $\tau_{0}$ are listed as follows,

$$
\begin{aligned}
& C_{n}=u_{n} C_{0}{ }^{t} u_{n}, \quad C_{n}^{\prime}=u_{n} C_{0}^{\prime t} u_{n}, \quad C_{n}^{\prime \prime}=u_{n} C_{0}^{\prime \prime} u_{n}, \quad u_{n}=\left(\begin{array}{ccc}
1 & 0 & 0 \\
0 & 1 & 0 \\
n_{1} & n_{2} & 1
\end{array}\right), \\
& \left(n_{i} \in Z\right) \\
& C_{0}=\left\{y=\left(y_{i j}\right) ; \begin{array}{l}
y_{11}+y_{12}+y_{13}>0, y_{12}+y_{22}+y_{23}>0 \\
y_{13}+y_{23}+y_{33}>0,-y_{12}>0,-y_{13}>0,-y_{23}>0
\end{array}\right\} \\
& C_{0}^{\prime}=\left\{y=\left(y_{i j}\right) ; \begin{array}{l}
y_{12}+y_{22}>0,+y_{13}+y_{23}>0,-y_{23}>0 \\
y_{11}+y_{12}-y_{13}-y_{23}>0,-y_{12}+y_{23}>0,-y_{13}+y_{33}>0
\end{array}\right\} \\
& C_{0}^{\prime \prime}=\left\{y=\left(y_{i j}\right) ; \begin{array}{l}
y_{11}+y_{12}>0, y_{13}+y_{23}>0,-y_{13}>0 \\
y_{12}+y_{22}-y_{13}-y_{23}>0,-y_{12}+y_{13}>0,-y_{23}+y_{33}>0
\end{array}\right\} \\
& X_{\tau_{0}} \cap \mathscr{X}_{C_{0}}^{0}=\left\{\begin{array}{c}
\left(x_{11} x_{12} x_{13}, x_{12} x_{22} x_{23}, x_{12}^{-1}, x_{13}^{-1}, x_{23}^{-1}\right) ; \\
\left(\begin{array}{cc}
\left(x_{11} x_{12} x_{13}\right)\left(x_{13}^{-1}\right), & x_{12} \\
x_{12} & \left(x_{12} x_{22} x_{23}\right)\left(x_{23}^{-1}\right)
\end{array}\right) \in T_{2}^{0}
\end{array}\right\}, \quad x_{i j}=e\left(\frac{1}{\lambda} \tau_{i j}\right)
\end{aligned}
$$

etc.

$X_{\tau_{0}}$ has a fibering over $\widetilde{\Xi}_{2}^{*}(\lambda)$ with principally polarized abelian varieties of $\operatorname{dim} 2$ as general fibers. Analytic subsets in $X_{\tau_{0}} \cap \mathscr{X}_{C_{0}}^{0}$ defined by $x_{13}, x_{23}=\lambda$-th root of unity and their transforms by $u_{n}$ give a level 
$\lambda$-structure on $X_{\tau_{0}}$.

Let $p$ be the point in $\widetilde{\varsigma}_{2}^{*}(\lambda)$ defined by

$$
s_{12}^{-1}=s_{11} s_{12}=s_{12} s_{22}=0 \text { in } \widetilde{\varsigma}_{2}^{*}(\lambda) \cap \mathscr{X}_{D_{0}} .
$$

Then the fiber at $p$ has the following configuration, $(\lambda \geqq 3)$

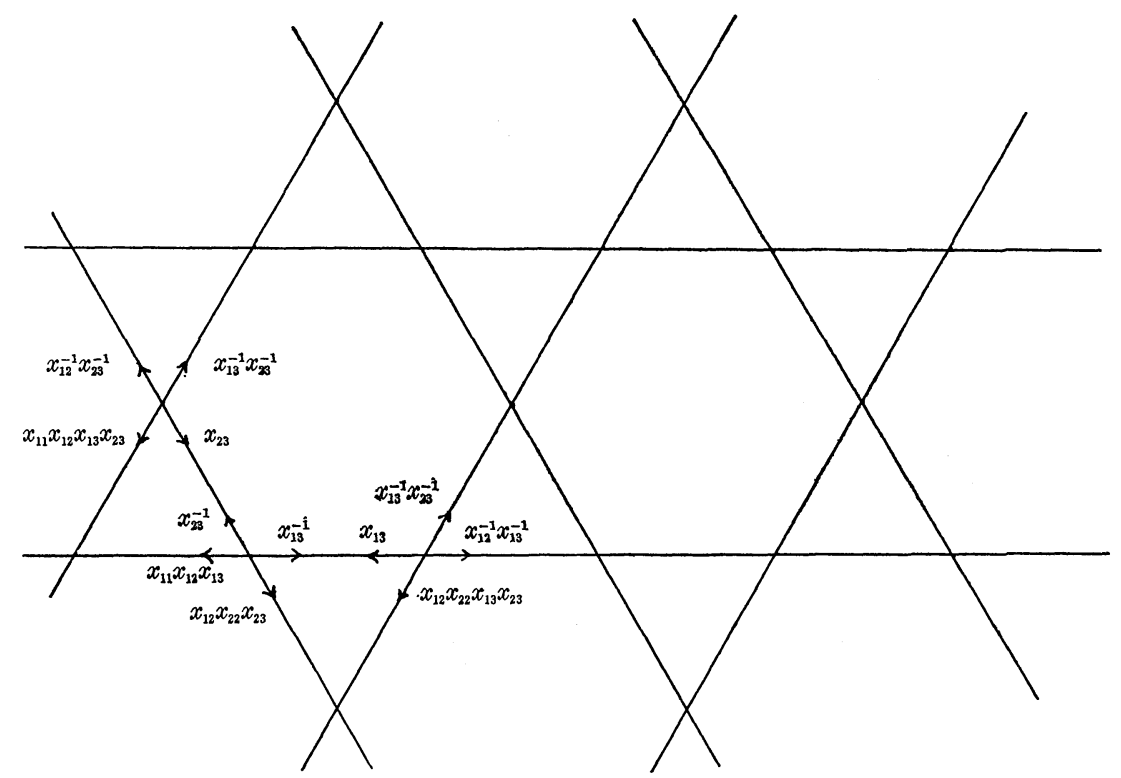

The fibering of $X_{\tau_{0}}$ near $p$ coincides with Deligne's example given in [13]. If $\lambda=2$, the fiber at $p$ consists of eight copies of a projective plane. If $\lambda=1$, the fiber at $p$ consists of two copies of a projective plane, which is nothing but our stable quasi abelian variety of level 1 . In the final section we shall give a brief account of the reason why we have taken our definition of stable quasi abelian varieties in spite of the above fact. In case of $g=4, X_{\tau_{0}}$ has also a fibering over $\widetilde{\Im}_{3}^{*}(\lambda)$, but the fibers are not equidimensional. The details will appear in the subsequent paper.

\section{§ 4. Infinitesimal deformations of SQAV (1)}

(4.1) For later use, we consider the following algebraic affine $C$ scheme $Y$ of $\operatorname{dim} 2$ defined by,

$$
\begin{aligned}
& Y=Y_{3}=\operatorname{Spec} C\left[x_{1}, x_{2}, x_{3}\right] /\left(x_{1} x_{2} x_{3}\right) \quad(n=3) \\
& Y=Y_{n}=\operatorname{Spec} C\left[x_{1}, \cdots, x_{n}\right] /\left(x_{i} x_{j}, j \neq i, i \pm 1 \bmod n\right) \quad(n>3) \\
& \quad \text { where } x_{n+i}=x_{i} .
\end{aligned}
$$


For example, $Y_{4}$ is an affine scheme Spec $C\left[x_{1}, x_{2}, x_{3}, x_{4}\right] /\left(x_{1} x_{3}, x_{2} x_{4}\right)$. We define

$$
\begin{array}{rlr}
D & =\operatorname{Spec} C\left[x_{1}, x_{2}, x_{2}\right] /\left(x_{1} x_{2}, x_{2} x_{3}, x_{3} x_{1}\right), \quad(n=3) \\
D^{\prime} & =\operatorname{Spec} C\left[x_{1}, x_{2}, x_{3}, x_{4}\right] /\left(x_{1} x_{3}, x_{2}, x_{4}\right) \quad(n=4) \\
D^{\prime \prime} & =\operatorname{Spec} C\left[x_{1}, x_{2}, x_{3}, x_{4}\right] /\left(x_{1}, x_{3}, x_{2} x_{4}\right) \quad(n=4) \\
D_{i} & =\operatorname{Spec} C\left[x_{1}, \cdots, x_{n}\right] /\left(x_{1}, \cdots, x_{i-1}, x_{i+1}, \cdots, x_{n}\right) \quad(n>4) .
\end{array}
$$

Then we have,

\section{LEMMA 4.1.}

(1) $\mathscr{E}_{x t^{1}}\left(\Omega_{Y}^{1} \mathcal{O}_{Y}\right)=\mathscr{O}_{D} \quad(n=3)$

(2) $\mathscr{E}_{x t^{1}}\left(\Omega_{Y}^{1} \mathcal{O}_{Y}\right)=\mathcal{O}_{D^{\prime}} \oplus \mathcal{O}_{D^{\prime \prime}} \quad(n=4)$

(3) $\mathscr{E}_{x t^{1}}\left(\Omega_{Y}^{1} \mathcal{O}_{Y}\right)=\bigoplus_{i=1}^{n} \mathcal{O}_{D_{i}} \quad(n>4)$

Proof. (1) and (2) are left to the readers. We prove the last assertion (3) only. At first we have the following exact sequence of $\mathscr{O}_{Y}$ modules

$$
\mathcal{O}_{Y}^{1 / 6 n(n+1)(n-4)} \stackrel{h_{2}}{\longrightarrow} \mathcal{O}_{Y}^{1 / 2 n(n-3)} \stackrel{h_{1}}{\longrightarrow} \mathcal{O}_{Y}^{n} \stackrel{h_{0}}{\longrightarrow} \Omega_{Y}^{1} \longrightarrow 0
$$

where $h_{\nu}$ is defined by,

$$
\begin{aligned}
& h_{0}\left(\left(a_{i}\right)_{i=1, \ldots, n}\right)=\sum_{i=1}^{n} a_{i} d x_{i}, \\
& h_{1}\left(\left(a_{i j}\right)\right)=\left(\sum_{j=1}^{n} a_{i j} x_{j}\right)_{i} ; a_{i j}=0 \quad(j=i, i \pm 1), a_{i j}=a_{j i}, \\
& h_{2}\left(\left(a_{i j}^{k}\right)\right)=\left(\sum_{k=1}^{n} a_{i j}^{k} x_{k}\right)_{i j} ; a_{i j}^{k}=0 \\
& \quad(j=i, i \pm 1), a_{i j}^{k}=a_{j i}^{k}, a_{i k}^{k+1}+a_{i k+1}^{k}=0, a_{i j}^{i}=0
\end{aligned}
$$

In fact, we have $h_{1} \circ h_{2}=0, h_{0} \circ h_{1}=0$ and $\operatorname{Ker} h_{0}=\operatorname{Im} h_{1}$ by definition. We shall show $\operatorname{Ker} h_{1}=\operatorname{Im} h_{2}$. Take an element $a_{i j}\left(a_{i j}=a_{j i}\right)$ of $\operatorname{Ker} h_{1}$. Then any $a_{i j}$ is contained in the maximal ideal $\sum_{j=1}^{n} x_{j} \mathcal{O}_{X}$, so we write $a_{i j}=\sum_{k=1}^{n} a_{i j}^{k} x_{k}$. We shall show that we can choose $a_{i j}^{k}$ in such a way that $a_{i j}^{k}=a_{j i}^{k}, a_{i j}^{i}=0, a_{i k}^{k+1}+a_{i k+1}^{k}=0, a_{i j}^{k}=0(j=i, i \pm 1)$. Since

$$
\begin{aligned}
\sum_{j=1}^{n} a_{i j} x_{j} & =\sum_{j=1}^{n}\left(a_{i j}^{j-1} x_{j-1} x_{j}+a_{i j}^{j} x_{j}^{2}+a_{i j}^{j+1} x_{j} x_{j+1}\right) \\
& =\sum_{j=1}^{n}\left(a_{i j}^{j+1}+a_{i j+1}^{j}\right) x_{j} x_{j+1}+\sum_{j=1}^{n} a_{i j}^{j} x_{j}^{2},
\end{aligned}
$$

we have on $X_{k}=\operatorname{Spec} C\left[x_{1}, \cdots, x_{n}\right] /\left(x_{j} j \neq k, k+1\right)$ 


$$
\left(a_{i k}^{k+1}+a_{i k+1}^{k}\right) x_{k} x_{k+1}+a_{i k}^{k} x_{k}^{2}+a_{i k+1}^{k+1} x_{k+1}^{2}=0 .
$$

Hence it follows $a_{i k}^{k} x_{k} \in x_{k-1} x_{k} \mathcal{O}_{X}+x_{k} x_{k+1} \mathcal{O}_{X}$ so that by a change of a choice of $\alpha_{i k}^{k-1}$ and $a_{i k}^{k+1}$ we may assume $a_{i k}^{k}=0$. Since $i \neq k-1, k+1$ (we notice that if $i=k \pm 1$, then $a_{i k}=0$ so that we may assume $a_{i k}^{j}=0$ from the beginning.), we can also choose $a_{i k}^{i-1}$ and $a_{i k}^{i+1}$ in such a way that $a_{i k}^{i}=0$. Consequently we obtain $\sum_{j=1}^{n} a_{i j} x_{j}=\sum_{j=1}^{n}\left(a_{i j}^{j+1}+a_{i j+1}^{j}\right) x_{j} x_{j+1}$. Then moreover we may assume $a_{i j}^{j+1}+a_{i j+1}^{j}=0$. In fact, $x_{k} x_{k+1}\left(\sum_{j=1}^{n} a_{i j} x_{j}\right)$ $=\left(a_{i k}^{k+1}+a_{i k+1}^{k}\right) x_{k}^{2} x_{k+1}^{2}=0$ hence $a_{i k}^{k+1}+a_{i k+1}^{k} \in \sum_{j \neq k, k+1} x_{j} \mathcal{O}_{X}$ so that we can write $a_{i k}^{k+1}+a_{i k+1}^{k}=\sum_{j \neq k, k+1} g_{j} x_{j}$. We change $a_{i k}^{k+1}$ and $a_{i k+1}^{k}$ into $a_{i k}^{k+1}+$ $\sum_{j \neq k, k+1, k+2} g_{j} x_{j}$ and $a_{i k+1}^{k}+g_{k+2} x_{k+2}$ respectively. Then we have $\left(a_{i k}^{k+1}+\right.$ $\left.\sum_{j \neq k, k+1, k+2} g_{j} x_{j}\right) x_{k+1}=a_{i k}^{k+1} x_{k+1}$ and $\left(a_{i k+1}^{k}+g_{k+2} x_{k+2}\right) x_{k}=a_{i k+1}^{k} x_{k}$. Thus we have proved the exactness of $\left(^{*}\right)$.

Next, dualizing $(*)$ by Hom $\left(\mathcal{O}_{X}\right)$, we obtain the following sequence

$$
\mathcal{O}_{X}^{n} \stackrel{h_{1}^{*}}{\longrightarrow} \mathcal{O}_{X}^{n(n-3) / 2} \stackrel{h_{2}^{*}}{\longrightarrow} \mathcal{O}_{X}^{n(n+1)(n-4) / 6},
$$

where $h_{\nu}^{*}$ is the dual of $h_{\nu}$, hence given by

$$
\begin{aligned}
h_{1}^{*}\left(\left(a_{i}^{*}\right)\right) & =\left(a_{i}^{*} x_{j}+a_{j}^{*} x_{i}\right)_{(i, j)} \\
h_{2}^{*}\left(\left(a_{i j}^{*}\right)\right) & =\left(a_{i j}^{* k}\right), \quad a_{i j}^{*}=a_{j i}^{*}, a_{i j}^{*}=0 \quad(j=i, i \pm 1) . \\
a_{i j}^{* k} & =a_{i j}^{*} x_{k} \\
a_{i j}^{* j+1} & =a_{i j}^{*} x_{j+1}-a_{i j+1}^{*} x_{j}
\end{aligned}
$$

By the same deduction we conclude that

$$
\operatorname{Ker} h_{2}^{*}=\left\{\begin{array}{l}
\left(a_{i j}^{*}\right) \in \mathcal{O}_{X}^{n(n-3) / 2} ; \\
a_{i j}^{*}=c_{j} x_{i}+c_{i} x_{j} \quad(j \neq i, i \pm 1, i+2) \\
a_{i i+2}^{*}=c_{i+2} x_{i}+c_{i} x_{i+2}+b_{i+1} x_{i+1} \quad c_{i} \in \mathcal{O}_{X}, b_{i} \in \mathcal{O}_{X}
\end{array}\right\}
$$

On the other hand, we infer $\operatorname{Im} h_{1}^{*}=\left\{\left(a_{i j}^{*}\right) ; a_{i j}^{*}=c_{i} x_{j}+c_{j} x_{i}\right\}$, hence $\mathscr{E}_{x t}{ }^{1}\left(\Omega_{X}^{1} \mathcal{O}_{X}\right)=\bigoplus_{i=1}^{n} \mathcal{O}_{D_{i}}$.

(4.2) The main purpose of this and next section is to prove the following

Theorem 4.1. Assume $X$ to be a SQAV of dimension $g(g=2,3)$ of level 1. Then we have,

$$
\operatorname{dim}_{C} \operatorname{Ext}^{1}\left(\Omega_{X}^{1} \mathcal{O}_{X}\right)=g^{2}
$$


We shall calculate $\operatorname{dim} \operatorname{Ext}^{1}\left(\Omega_{X}^{1} \mathcal{O}_{X}\right)$ by use of the following spectral sequence,

$$
E_{2}^{p g}=H^{p}\left(X, \mathscr{E}_{x t^{q}}\left(\Omega_{X}^{1} \mathcal{O}_{X}\right)\right) \rightarrow \operatorname{Ext}^{p+q}\left(\Omega_{X}^{1} \mathcal{O}_{X}\right)
$$

Our idea is to compute the two terms $H^{0}\left(X, \mathscr{E} x t^{1}\left(\Omega_{X}^{1} \mathcal{O}_{X}\right)\right)$, and $H^{0}\left(X, \mathscr{E} x t^{0}\left(\Omega_{X}^{1} \mathcal{O}_{X}\right)\right)$ and show $\operatorname{dim} H^{0}\left(X, \mathscr{E}_{x t} t^{1}\left(\Omega_{X}^{1} \mathcal{O}_{X}\right)\right)+\operatorname{dim} H^{1}\left(X, \mathscr{E} x t^{0}\left(\Omega_{X}^{1} \mathcal{O}_{X}\right)\right)$ $\leqq g^{2}$ if $g=2,3$. If this is done, we have $\operatorname{dim} \operatorname{Ext}^{1}\left(\Omega_{X}^{1} \mathcal{O}_{X}\right) \leqq g^{2}$, while using the explicit structure of $\mathrm{D}-\mathrm{V}$ cones we can construct a flat family of not necessarily polarized deformations of $X$ depending on $g^{2}$ effective parameters, so that we have $\operatorname{dim} \operatorname{Ext}^{1}\left(\Omega_{X}^{1} \mathcal{O}_{X}\right)=g^{2}$.

In case of $g=2$, all the possible SQAV's of level 1 are listed in the following table.

(1) non singular principally polarized abelian surface

(2) a $P^{1}$-bundle over an elliptic curve glued along 0 -section and $\infty$ section

(3) $P^{1} \times P^{1}$ glued along two pairs of opposite lines.

(4) two copies of $P^{2}$ glued along three pairs of lines.

In what follows, we simply denote by $X_{\nu}$ the $\nu$-th SQAV, for instance, by $X_{1}$ a non-singular abelian surface etc.

case 1)

LEMMA 4.2. $\operatorname{dim} \operatorname{Ext}^{1}\left(\Omega_{X_{1}}^{1} \mathcal{O}_{X_{1}}\right)=4$

Proof. well known.

case 2)

$X_{2}$ is isomorphic to the closed fiber of $\operatorname{Proj} R(\tau) / \Gamma$ where $\tau(s)=$ $\left(\begin{array}{ll}\tau_{11} & \tau_{12} \\ \tau_{12} & \tau_{22}\end{array}\right), \tau_{22}=\log s / 2 \pi \sqrt{-1}$, and $\tau_{11}, \tau_{12}$ : constant. The normalization $\tilde{X}_{2}$ of $X_{2}$ is a $P^{1}$ bundle over an elliptic curve $E$ with periods $1, \tau_{11}$, which is a compactification of $C^{*}$ bundle over $E$ whose corresponding one cocyle is $e\left(\tau_{12}\right)$ in $\operatorname{Ext}^{1}\left(E, C^{*}\right) \cong E$ through a canonical isomorphism. $X_{2}$ is obtained from $\tilde{X}_{2}$ by identifying 0 section and $\infty$ section by the relation $(z, 0) \equiv\left(z+\tau_{12}, \infty\right)$. We denote by $C$ the double curve in $X_{2}$. It is obvious that $C$ is isomorphic with $E$, and a long $C, X_{2}$ is described by;

$$
X_{2}=\{(x, y, z) ; x y=0, z \in E\} \text {. }
$$

The semi universal covering $P_{0}$ of $X$ is covered with $V_{i}, i \in Z$, and $V_{i}$ is locally given by $\left\{\left(x_{i}, y_{i}, z\right), x_{i} y_{i}=0, z \in E\right\}$ along $C_{i}=\left\{x_{i}=y_{i}=0\right\}$ 
LEMMA 4.3.

(1) $0 \longrightarrow \mathscr{E} x t^{0}\left(\Omega_{X}^{1} \mathcal{O}_{X}\right) \stackrel{\iota}{\longrightarrow} \mathcal{O}_{\tilde{X}}^{\oplus 2} \stackrel{\psi_{0}}{\longrightarrow} \mathcal{O}_{C} \longrightarrow 0 \quad$ (exact)

(2) $\operatorname{Ext}^{1}\left(\Omega_{X}^{1} \mathcal{O}_{X}\right) \cong \mathcal{O}_{C}$.

where in (1), the first homomorphism c is given by

$$
\begin{aligned}
\iota\left(a_{0}(x, y, z) \frac{\partial}{\partial z}+a_{1}(x, y, z) x \frac{\partial}{\partial x}+a_{2}(x, y, z) y \frac{\partial}{\partial y}\right. \\
\quad=\left(a_{0}(x, 0, z)+a_{1}(x, 0, z), a_{0}(0, y, z)+a_{2}(0, y, z)\right)
\end{aligned}
$$

and also $\psi_{0}$ is defined by

$$
\psi_{0}\left(\left(a_{0}(x, z), a_{1}(x, z)\right) \oplus\left(a_{0}^{\prime}(y, z), a_{2}(y, z)\right)\right)=a_{0}(0, z)-a_{0}^{\prime}(0, z) .
$$

Proof. The exactness of (1) follows directly from the above definitions of $\iota, \psi_{0}$. On $P_{0}=(\operatorname{Proj} R)_{0}$, there exists an isomorphism $\mathscr{E} x t^{1}\left(\Omega_{P_{0}}^{1} \mathcal{O}_{P_{0}}\right)$ $=\oplus_{i=-\infty}^{\infty} \mathcal{O}_{C_{i}}$. This isomorphism is deduced from the complex,

$$
\mathcal{O}_{P_{0}}^{\oplus 3} \stackrel{h_{1}^{*}}{\longrightarrow} \mathcal{O}_{P_{0}} \stackrel{h_{2}^{*}}{\longrightarrow} 0
$$

obtained by dualizing an exact sequence of $\mathcal{O}_{P_{0}}$ modules,

$$
0 \stackrel{h_{2}}{\longrightarrow} \mathcal{O}_{P_{0}} \stackrel{h_{1}}{\longrightarrow} \mathcal{O}_{P_{0}}^{\oplus 3} \stackrel{h_{0}}{\longrightarrow} \mathcal{O}_{P_{0}} \longrightarrow 0 \text {. }
$$

In the last sequence $h_{0}, h_{1}$ and $h_{2}$ are defined by

$$
\begin{gathered}
h_{0}\left(\left(a_{1}, a_{2}, a_{3}\right)\right)=a_{1} d z+a_{2} d x_{i}+a_{3} d y_{i} \\
h_{1}\left(a_{4}\right)=\left(0, a_{4} y_{i}, a_{4} x_{i}\right), \quad h_{2}=0 .
\end{gathered}
$$

The isomorphism between $\operatorname{Ker} h_{2}^{*} / \operatorname{Im} h_{1}^{*}$ and $\bigoplus_{i=-\infty}^{\infty} \mathcal{O}_{C_{i}}$ is invariant under the action of $\Gamma$, so we have our lemma.

Q.E.D.

From Lemma 4.3., it follows,

$$
\operatorname{dim} H^{0}\left(\mathscr{E} x t^{1}\left(\Omega_{X}^{1} \mathcal{O}_{X}\right)\right)=1
$$

and

$$
\begin{aligned}
0 & \rightarrow H^{0}\left(\mathscr{E} x t^{0}\left(\Omega_{X}^{1} \mathcal{O}_{X}\right)\right) \rightarrow C \oplus C \rightarrow C \\
& \rightarrow H^{1}\left(\mathscr{E} x t^{0}\left(\Omega_{X}^{1} \mathcal{O}_{X}\right)\right) \rightarrow C \oplus C \rightarrow 0 \\
& \rightarrow H^{2}\left(\mathscr{E} x t^{0}\left(\Omega_{X}^{1} \mathcal{O}_{X}\right)\right) \rightarrow 0
\end{aligned}
$$

so that we obtain, $\operatorname{dim} H^{0}\left(\mathscr{E} x t^{0}\left(\Omega_{X}^{1} \mathcal{O}_{X}\right)\right)=2, \operatorname{dim} H^{1}\left(\mathscr{E} x t^{0}\left(\Omega_{X}^{1} \mathcal{O}_{X}\right)\right)=3$ and 
$\operatorname{dim} H^{2}\left(\mathscr{E} x t^{0}\left(\Omega_{X}^{1} \mathcal{O}_{X}\right)\right)=0$.

In this case the spectral sequence $E_{2}^{p q}=H^{p}\left(X, \mathscr{E}_{x t^{q}}\left(\Omega_{X}^{1} \mathcal{O}_{X}\right)\right)$ gives the exact dimension of $\operatorname{Ext}^{1}\left(\Omega_{X}^{1} \mathcal{O}_{X}\right)$ as follows; the sequence

$$
0 \rightarrow E_{2}^{1,0} \rightarrow \mathscr{E}_{x t^{1}}\left(\Omega_{X}^{1} \mathcal{O}_{X}\right) \rightarrow E_{2}^{0,1} \rightarrow E_{2}^{2,0}=0
$$

is exact, so we have $\operatorname{dim} \operatorname{Ext}^{1}\left(\Omega_{X}^{1} \mathcal{O}_{X}\right)=4$.

Remark. The geometric meaning of the above computation is accounted for by considering that $\operatorname{dim} H^{0}\left(\mathscr{E}_{x t} t^{1}\left(\Omega_{X}^{1} \mathcal{O}_{X}\right)\right)$ is the number of parameters deforming the singular loci of $X$, and $\operatorname{dim} H^{1}\left(\mathscr{E} x t^{0}\left(\Omega_{X}^{1} \mathcal{O}_{X}\right)\right)$ is the sum of the numbers of parameters of the moduli of the normalization $\tilde{X}_{2}$ and parameters of gluing $\tilde{X}_{2}$ into $X_{2}$. case 3)

$X_{3}$ is isomorphic to the closed fiber of $\operatorname{Proj} R(\tau) / \Gamma$ with $\tau(s)=$ $\left(\begin{array}{ll}\tau_{11} & \tau_{12} \\ \tau_{12} & \tau_{22}\end{array}\right), \tau_{11}=\tau_{22}=\log s / 2 \pi \sqrt{-1}, \tau_{12}:$ constant.
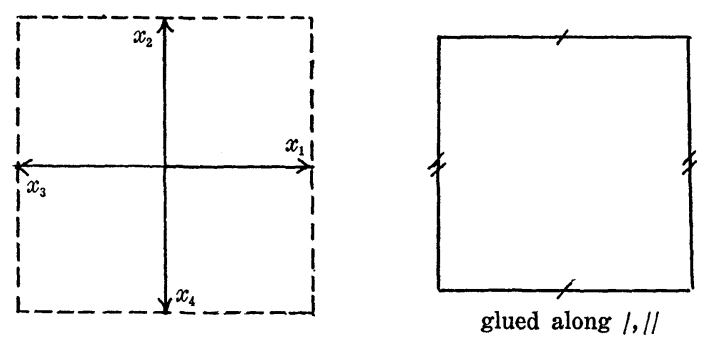

At $p, X_{3}$ is locally isomorphic to Spec $C\left[x_{1}, x_{2}, x_{3}, x_{4}\right] /\left(x_{1} x_{3}, x_{3} x_{4}\right) . \quad X_{3}$ has two double curves $C_{1}, C_{2}$ which are rational curves with one ordinally double point respectively.

The normalization $\tilde{X}_{3}$ of $X_{3}$ is $P^{1} \times P^{1} . \quad X_{3}$ is obtained from $\tilde{X}_{3}$ by identifying two pair of opposite lines by the relations,

$$
\left(x_{1}, 0\right) \equiv\left(e\left(\tau_{12}\right) x_{1}, \infty\right), \quad\left(0, x_{2}\right) \equiv\left(\infty, e\left(\tau_{12}\right) x_{2}\right)
$$

where $\left(x_{1}, x_{2}\right)$ is the usual inhomogeneous coordinate of $P^{1} \times P^{1}$. Then we have,

LEMMA 4.4.

(1) $0 \longrightarrow \mathscr{E} x t^{0}\left(\Omega_{X}^{1} \mathcal{O}_{X}\right) \stackrel{\iota}{\longrightarrow} \mathcal{O}_{\tilde{X}_{3}}^{\oplus 2} \stackrel{\psi_{0}}{\longrightarrow} \mathcal{O}_{\tilde{C}_{1}} \oplus \mathcal{O}_{\tilde{C}_{2}} \longrightarrow 0 \quad$ (exact)

(2) $\mathscr{E}_{x t^{1}}\left(\Omega_{X}^{1} \mathcal{O}_{X}\right) \cong \mathcal{O}_{C_{1}} \oplus \mathcal{O}_{C_{2}}$ 
where $\tilde{C}_{i}$ denotes the normalization of $C_{i}$ and $\iota, \psi_{0}$ are given by at $p=$ $\left(x_{1}, x_{2}, x_{3}, x_{4}\right)=(0,0,0,0)$

$$
\begin{aligned}
& \iota\left(\sum_{i=1}^{4} a_{i}(x) x_{i} \frac{\partial}{\partial x_{i}}\right)=\left(a_{i}\left(x_{i}, x_{i+1}\right)+a_{i+1}\left(x_{i}, x_{i+1}\right)\right)_{i=1, \ldots, 4} \\
& \psi_{0}\left(\left(a_{i}^{\prime}\left(x_{i}, x_{i+1}\right)+a_{i}^{\prime \prime}\left(x_{i}, x_{i+1}\right)\right)=\left(\alpha_{i}^{\prime \prime}\left(0, x_{i+1}\right)-a_{i+1}^{\prime}\left(x_{i+1}, 0\right)_{i=1, \ldots, 4}\right.\right.
\end{aligned}
$$

and we put $a_{i}(x)=a_{i}\left(x_{1}, x_{2}, x_{3}, x_{4}\right), a_{i}\left(x_{j}, x_{j+1}\right)=a_{i}\left(0 \cdots 0, x_{j}, x_{j+1}, 0 \cdots 0\right)$, $x_{5}=x_{1}$.

Proof. (1) follows directly from the above definition.

(2) is proved in the same way as before by virtue of Lemma 4.1.

Q.E.D.

According to Lemma 4.4., it follows

$$
\begin{array}{rlrl}
\operatorname{dim} H^{0}\left(X, \mathscr{E} x t^{0}\left(\Omega_{X}^{1} \mathcal{O}_{X}\right)\right) & =2, & & \operatorname{dim} H^{1}\left(X, \mathscr{E} x t^{0}\left(\Omega_{X}^{1} \mathcal{O}_{X}\right)\right)=2 \\
\operatorname{dim} H^{2}\left(X, \mathscr{E} x t^{0}\left(\Omega_{X}^{1} \mathcal{O}_{X}\right)\right)=0, & & \operatorname{dim} H^{0}\left(X, \mathscr{E} x t^{1}\left(\Omega_{X}^{1} \mathcal{O}_{X}\right)\right)=2 .
\end{array}
$$

Hence we have $\operatorname{dim} \operatorname{Ext}^{1}\left(\Omega^{1}{ }_{X} \mathcal{O}_{X}\right)=4$ in the same way as in the case (2).

case 4)

$X_{4}$ is isomorphic to the closed fiber of $\operatorname{Proj} R(\tau) / \Gamma$ with $\tau=$ $\left(\begin{array}{rr}2 & -1 \\ -1 & 2\end{array}\right) \log s / 2 \pi \sqrt{-1} . \quad X_{4}$ is a union of two copies of the projective plane $P_{2}$, has three double curves $C_{i}(i=1,2,3) . \quad C_{i}$ is also a rational curve with one ordinary double point, and all $C_{i}$ meet at the unique point $p$, and there $X_{4}$ is locally isomorphic to $Y_{6}$ given in (4.1). Denoting by $\tilde{C}_{i}$ the normalization of $C_{i}(i=1,2,3)$ we have,

\section{LEMMA 4.5.}

(1) $\quad 0 \longrightarrow \mathscr{E} x t^{0}\left(\Omega_{X}^{1} \mathcal{O}_{X}\right) \stackrel{\iota}{\longrightarrow} \mathcal{O}_{\tilde{X}}^{\oplus 2} \stackrel{\psi_{0}}{\longrightarrow} \bigoplus_{i=0}^{3} \mathcal{O}_{\tilde{C}_{i}} \longrightarrow 0$

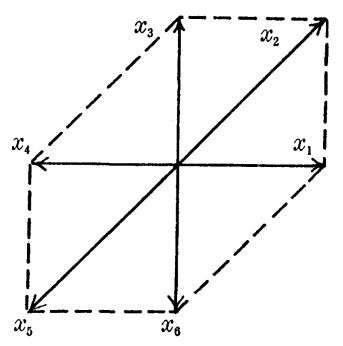


(2) $\mathscr{E}_{x t^{1}}\left(\Omega_{X}^{1} \mathcal{O}_{X}\right)=\oplus_{i=1}^{3} \mathcal{O}_{\mathcal{O}_{i}}$

where $c$ and $\psi_{0}$ are defined by,

$$
\begin{gathered}
\iota\left(\sum_{i=1}^{6} a_{i}(x) x_{i} \frac{\partial}{\partial x_{i}}\right)=\left(a_{i}\left(x_{i}, x_{i+1}\right)+a_{i+1}\left(x_{i}, x_{i+1}\right)\right)_{i=1, \ldots, 6}, \\
a_{i}(x)=a_{i}\left(x_{1}, \cdots, x_{6}\right) \quad \text { and } \quad a_{i}\left(x_{j}, x_{j+1}\right)=a_{i}\left(0 \ldots 0, x_{j}, x_{j+1}, 0 \cdots 0\right) \\
\psi_{0}\left(\left(a_{i}^{\prime}\left(x_{i}, x_{i+1}\right) \oplus a_{i}^{\prime \prime}\left(x_{i}, x_{i+1}\right)\right)=\left(a_{i}^{\prime \prime}\left(0, x_{i+1}\right)-a_{i+1}^{\prime}\left(x_{i+1}, 0\right)\right)_{i=1, \ldots, 6} .\right.
\end{gathered}
$$

We omit the proof of Lemma. From this lemma, we infer that

$$
\begin{array}{ll}
\operatorname{dim} H^{0}\left(X, \mathscr{E} x t^{1}\left(\Omega_{X}^{1} \mathcal{O}_{X}\right)\right)=3, & \operatorname{dim} H^{0}\left(X, \mathscr{E} x t^{0}\left(\Omega_{X}^{1} \mathcal{O}_{X}\right)\right)=2, \\
\operatorname{dim} H^{1}\left(X, \mathscr{E} x t^{0}\left(\Omega_{X}^{1} \mathcal{O}_{X}\right)\right)=1, & \operatorname{dim} H^{2}\left(X, \mathscr{E} x t^{0}\left(\Omega_{X}^{1} \mathcal{O}_{X}\right)\right)=0 .
\end{array}
$$

In fact, for instance, $H^{1}\left(X, \mathscr{E}_{x t^{0}}\left(\Omega_{X}^{1} \mathcal{O}_{X}\right)\right)$ is calculated as follows. We fix an orientation of each Delone cell as described in the picture. Then $H^{1}\left(X \mathscr{E}_{x t}{ }^{0}\left(\Omega_{X}^{1} \mathcal{O}_{X}\right)\right)$ is given by,

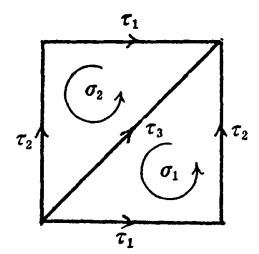

$$
\operatorname{Ker} \psi_{0} / \operatorname{Im} \iota=\left\{\left(C_{\tau_{j}}\right)_{j=1,2,3}\right\} /\left\{C_{\tau_{j}}=\Sigma_{i}\left[\sigma_{i}: \tau_{j}\right] C_{\left.\sigma_{i \tau}\right\}}\right\}
$$

where

$$
C_{\sigma i \tau_{3}}=C_{\sigma_{i \tau_{1}}}+C_{\sigma_{i \tau_{2}}}, \quad C_{\tau_{j}}, \quad C_{\sigma_{i \tau_{j}}} \in \boldsymbol{C},
$$

Hence $\operatorname{dim} H^{1}\left(X, \mathscr{E}_{x t^{0}}\left(\Omega_{X}^{1} \mathcal{O}_{X}\right)\right)=1$.

For the completeness of arguments we shall construct a "complete" flat family of deformations of $X$ depending on 4 effective parameters. We mean by "complete" that the Kodaira-Spencer mapping is surjective (see $\S 5$, [27]). Let $\tau(s)=\left(\begin{array}{ll}1 & 0 \\ 0 & 0\end{array}\right) \log s_{11} / 2 \pi \sqrt{-1}+\left(\begin{array}{ll}0 & 0 \\ 0 & 1\end{array}\right) \log s_{22} / 2 \pi \sqrt{-1}+$ $\left(\begin{array}{rr}1 & -1 \\ -1 & 1\end{array}\right) \log s_{12} / 2 \pi \sqrt{-1}+\left(\begin{array}{cc}0 & 0 \\ s_{21} & 0\end{array}\right)$ and $\mathscr{X}=\operatorname{Proj} \tilde{R}(\tau) / \Gamma$ where $\tilde{R}(\tau)=$ $\left[\tilde{\xi}_{m} \cdot \theta, m \in Z^{g}\right], \tilde{\xi}_{m}=e\left(\frac{1}{2} m \tau(s)^{t} m+m^{t}\left(z+\frac{1}{2} r(s)\right), r(s)=\left(\log s_{11}+\log s_{12} / 2 \pi \sqrt{-1}\right.\right.$, $\left.\log s_{12}+\log s_{22} / 2 \pi \sqrt{-1}\right) \mathcal{O}=\mathcal{O}_{0, D_{\varepsilon}}, D_{\varepsilon}=\left\{\left(s_{i j}\right) ;\left|s_{i j}\right|<\varepsilon\right\}$

Notice that ${ }^{t} \tau(s) \neq \tau(s)$. However the quotient of $\operatorname{Proj} \tilde{R}$ by $\Gamma$ really exists and the general fiber is a complex torus with period $(1, \tau(s))$ and the fiber $\mathscr{X}_{0}$ at the origin is $X$. 
In order to show the completeness of this family, we investigate the structure along the singular loci $X_{\tau_{i}}\left(=C_{i}\right)(i=1,2,3)$ of $X . P=\operatorname{Proj} \tilde{R}(\tau)$ is covered with open affines $U_{k}, k \in Z^{2}$, where

$$
U_{k}=\operatorname{Spec} \mathcal{O}\left[\tilde{\xi}_{m} \tilde{\xi}_{k}^{-1}, m \in Z^{2}\right] .
$$

We have only to see $U_{0}$. By an explicit calculation $U_{0}$ is given by,

$$
U_{0} \cong \operatorname{Spec} \mathcal{O}\left[w_{1}^{-1}, w_{2}^{-1}, s_{12}^{-1} w_{1}^{-1} w_{2}^{-1}, s_{11} s_{12} w_{1}, s_{12} s_{22} w_{2}, s_{11} s_{12} s_{22} w_{1} w_{2}\right]
$$

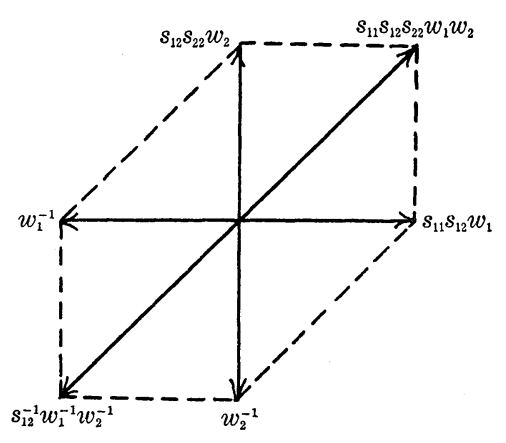

At the generic point of $X_{\tau_{1}}$, we have $s_{11} s_{12} w_{1} \neq 0$

$$
s_{22}=\left(s_{11} s_{12} w_{1}\right)^{-1}\left(s_{11} s_{12} s_{22} w_{1} w_{2}\right) w_{2}^{-1} .
$$

Hence $U_{0}$ is locally isomorphic to

$$
\text { Spec } C\left[s_{11}, s_{12}, s_{21}, s_{11} s_{12} w_{1},\left(s_{11} s_{12} w_{1}\right)^{-1}, w_{2}^{-1}, s_{11} s_{12} s_{22} w_{1} w_{2}\right]
$$

Hence $\partial / \partial s_{22}$ is mapped onto $H^{0}\left(X \mathcal{O}_{X_{\tau_{1}}}\right)\left(\subset H^{0}\left(X, \mathscr{E} x t^{1}\left(\Omega_{X}^{1} \mathcal{O}_{X}\right)\right)\right.$. In the same way, it is shown that $\partial / \partial s_{11}, \partial / \partial s_{12}, \partial / \partial s_{22}$ are mapped into a basis of $H^{0}\left(X, \mathscr{E} x t^{1}\left(\Omega_{X}^{1} \mathcal{O}_{X}\right)\right)$. On the other hand, $\partial / \partial s_{21}$ is mapped into a basis of $H^{1}\left(X, \mathscr{E} x t^{0}\left(\Omega_{X}^{1} \mathcal{O}_{X}\right)\right)$. Thus "completeness" is proved. ([27] theorem 3.5.)

Summing up the above calculations we obtain,

THEOREM 4.1. $\operatorname{dim} \operatorname{Ext}^{1}\left(\Omega_{X}^{1} \mathcal{O}_{X}\right)=4$ for a $S Q A V X$ of 2 dimension of level 1.

\section{§ 5. Infinitesimal deformations of SQAV (2)}

(5.1) At first, we shall give the complete list of $\mathrm{D}-\mathrm{V}$ cones in $\overline{\mathfrak{Y}}_{3}^{+}$ (up to $G L(3 Z)$ equivalence and the associated Delone decompositions (Voronoi [26]). D-V cones : 


$$
\begin{aligned}
& \Sigma_{1}=\left\{\left(\begin{array}{ccc}
0 & 0 & 0 \\
0 & 0 & 0 \\
0 & 0 & y_{33}
\end{array}\right) ; y_{33}>0\right\}, \quad \Sigma_{2}=\left\{\left(\begin{array}{ccc}
0 & 0 & 0 \\
0 & y_{22} & 0 \\
0 & 0 & y_{33}
\end{array}\right) ; y_{22}>0, y_{33}>0\right\} \\
& \Sigma_{3}=\left\{\left(\begin{array}{ccc}
0 & 0 & 0 \\
0 & y_{22} & y_{23} \\
0 & y_{23} & y_{33}
\end{array}\right) ; \begin{array}{r}
y_{22}+y_{23}>0 \\
y_{23}+y_{33}>0
\end{array}\right\}, \quad \Sigma_{4}=\left\{\left(\begin{array}{ccc}
y_{11} & 0 & 0 \\
0 & y_{22} & 0 \\
0 & 0 & y_{33}
\end{array}\right) ; y_{i i}>0\right\} \\
& \Sigma_{5}=\left\{\left(\begin{array}{ccc}
y_{11} & 0 & 0 \\
0 & y_{22} & y_{23} \\
0 & y_{23} & y_{33}
\end{array}\right) ; \quad \begin{array}{r}
y_{11}>0, y_{22}+y_{23}>0 \\
-y_{23}>0, y_{23}+y_{33}>0
\end{array}\right\} \\
& \left.\Sigma_{6}=\left\{\begin{array}{lll}
y_{11} & y_{12} & 0 \\
y_{12} & y_{22} & y_{23} \\
0 & y_{23} & y_{33}
\end{array}\right) ; \begin{array}{l}
y_{11}+y_{12}>0,-y_{12}>0 \\
y_{12}+y_{22}+y_{23}=0,-y_{23}>0 \\
y_{23}+y_{33}>0
\end{array}\right\} \\
& \left.\Sigma_{7}=\left\{\begin{array}{ccc}
y_{11} & y_{12} & 0 \\
y_{12} & y_{22} & y_{23} \\
0 & y_{23} & y_{33}
\end{array}\right) ; \begin{array}{l}
y_{11}+y_{12}>0,-y_{12}>0,-y_{23}>0 \\
y_{12}+y_{22}+y_{23}>0, y_{23}+y_{33}>0
\end{array}\right\} \\
& \left.\Sigma_{8}=\left\{\begin{array}{lll}
y_{11} & y_{12} & y_{13} \\
y_{12} & y_{22} & y_{23} \\
y_{13} & y_{23} & y_{33}
\end{array}\right) ; \begin{array}{cl}
\sum_{j=1}^{3} y_{i j}>0 & (i=1,2,3) \\
-y_{i j}>0 & (i \neq j)
\end{array}\right\}
\end{aligned}
$$

Associated Delone decompositions. (For brevity we only write representatives of Delone cells modulo translations by integral vectors)

Remark. In case of $\Sigma_{4}$, only one cube appears. In case of $\Sigma_{5}$ two triagonal prisms appear. In case of $\Sigma_{6}$, the shaded face is not a Delone cell, and all the Delone cells of dimension 3 modulo translations are two tetrahedrons and an octahedron which is a union of separated two copies
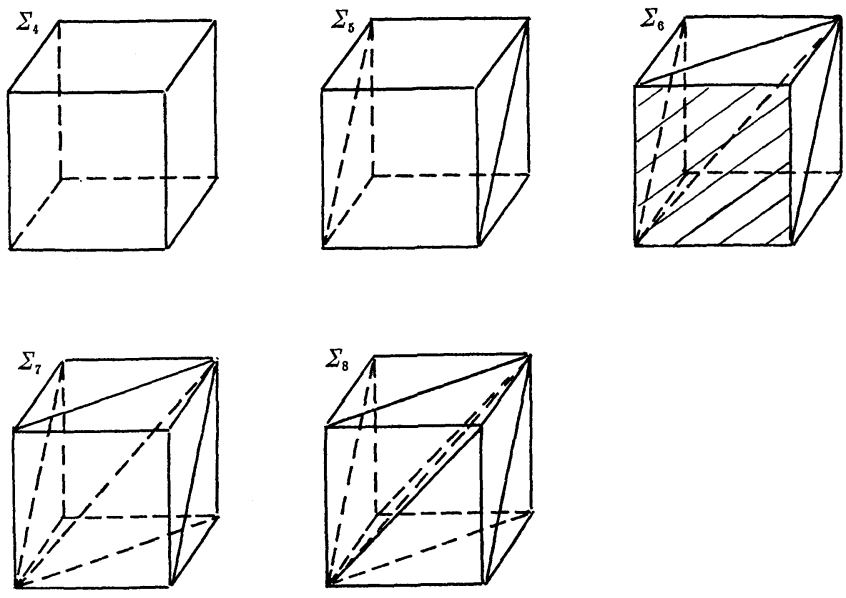
of quadrangular pyramid. On the contrary in case of $\Sigma_{7}$ two quadrangular pyramids and two tetrahedrons are all the Delone cells of dimension 3 although the picture looks like that in case of $\Sigma_{6}$. In the last case six tetrahedrons are all the Delone cells.

(5.2) For $\mathrm{D}-\mathrm{V}$ cones $\Sigma_{i}(i=1,2,3)$ the calculation of $\operatorname{Ext}^{1}\left(\Omega_{X}^{1} \mathcal{O}_{X}\right)$ can be carried out in the same way as before, so we omit the details and state the results only. We note that we restrict ourselves to the case $\lambda=1$.

case $\left.\Sigma_{1}\right) \quad \tau(s)=\left(\begin{array}{ccc}\tau_{11} & \tau_{12} & \tau_{13} \\ \tau_{12} & \tau_{22} & \tau_{23} \\ \tau_{13} & \tau_{23} & 0\end{array}\right)+\left(\begin{array}{ccc}0 & 0 & 0 \\ 0 & 0 & 0 \\ 0 & 0 & 1\end{array}\right) \log s / 2 \pi \sqrt{-1}$

with constant $\tau_{i j}((i j) \neq(33))$

$$
\begin{array}{ll}
H^{0}\left(X, \mathscr{E} x t^{0}\left(\Omega_{X}^{1} \mathcal{O}_{X}\right)\right)=3, & H^{1}\left(X, \mathscr{E}_{x t}{ }^{0}\left(\Omega_{X}^{1} \mathcal{O}_{X}\right)\right)=8 \\
H^{2}\left(X, \mathscr{E} x t^{0}\left(\Omega_{X}^{1} \mathcal{O}_{X}\right)\right)=7, & H^{0}\left(X, \mathscr{E}_{x t}{ }^{1}\left(\Omega_{X}^{1} \mathcal{O}_{X}\right)\right)=1
\end{array}
$$

Although $H^{2}\left(X, \mathscr{E} x t^{0}\left(\Omega_{X}^{1} \mathcal{O}_{X}\right)\right)$ does not vanish we can conclude $\operatorname{dim} \operatorname{Ext}^{1}\left(\Omega_{X}^{1} \mathcal{O}_{X}\right)$ $=9$ from the existence of a flat family of deformations of $X$ depending on 9 effective parameters.

case $\left.\Sigma_{2}\right) \quad \tau(s)=\left(\begin{array}{ccc}\tau_{11} & \tau_{12} & \tau_{13} \\ \tau_{12} & 0 & \tau_{23} \\ \tau_{13} & \tau_{23} & 0\end{array}\right)+\left(\begin{array}{ccc}0 & 0 & 0 \\ 0 & 1 & 0 \\ 0 & 0 & 1\end{array}\right) \log s / 2 \pi \sqrt{-1}$ with constant

Let $X$ be the closed fiber of $\operatorname{Proj}(R(\tau)) / \Gamma$. Then we have

$$
\begin{aligned}
& \operatorname{dim} H^{0}\left(X, \mathscr{E} x t^{0}\left(\Omega_{X}^{1} \mathcal{O}_{X}\right)\right)=3, \quad \operatorname{dim} H^{1}\left(X, \mathscr{E} x t^{0}\left(\Omega_{X}^{1} \mathcal{O}_{X}\right)\right)=7, \\
& \operatorname{dim} H^{0}\left(X, \mathscr{E} x t^{1}\left(\Omega_{X}^{1} \mathcal{O}_{X}\right)\right)=2 .
\end{aligned}
$$

Also in this case by constructing a flat family of deformations of $X$ depending on 9 effective parameters we obtain $\operatorname{dim} \operatorname{Ext}^{1}\left(\Omega_{X}^{1} \mathcal{O}_{X}\right)=9$

case $\left.\Sigma_{3}\right) \quad \tau(s)=\left(\begin{array}{ccc}\tau_{11} & \tau_{12} & \tau_{13} \\ \tau_{12} & 0 & 0 \\ \tau_{13} & 0 & 0\end{array}\right)+\left(\begin{array}{rrr}0 & 0 & 0 \\ 0 & 2 & -1 \\ 0 & -1 & 2\end{array}\right) \log s / 2 \pi \sqrt{-1}$.

Then similarly we have

$$
\begin{aligned}
& \operatorname{dim} H^{0}\left(X, \mathscr{E} x t^{0}\left(\Omega_{X}^{1} \mathcal{O}_{X}\right)\right)=3, \quad \operatorname{dim} H^{1}\left(\mathscr{E} x t^{0}\left(\Omega_{X}^{1} \mathcal{O}_{X}\right)\right)=6 \\
& \operatorname{dim} H_{0}\left(X, \mathscr{E} x t^{1}\left(\Omega_{X}^{1} \mathcal{O}_{X}\right)\right)=3, \quad \operatorname{dim} \operatorname{Ext}^{1}\left(\Omega_{X}^{1} \mathcal{O}_{X}\right)=9 .
\end{aligned}
$$

(5.3) Before we enter into the computation of $\operatorname{Ext}^{1}\left(\Omega_{X}^{1} \mathcal{O}_{X}\right)$ in case 
of $\Sigma_{i}(i \geqq 4)$, we introduce here a concept of a graph associated with SQAV $X$ as follows. A vertex denotes a subscheme of codimension one contained in the singular loci of $X$. Of course the latter corresponds to a Delone cell of codimension one, so we may say a graph corresponds to a Delone decomposition. Two or three vertices are connected by arrows only if

(i) If $X$ is locally given by Spec $C\left[x_{1}, x_{2}, x_{3}, x_{4}, z\right] /\left(x_{1} x_{3}, x_{2} x_{4}\right)$ along $Z=\operatorname{Spec} C[z]$ then we connect a vertex corresponding to $D_{1}$ with one corresponding to $D_{3}$, and do so for $D_{2}$ and $D_{4}$, where $D_{i}=\operatorname{Spec} C\left[x_{i}, z\right]$, $z=\left(z_{1}, \cdots, z_{g-2}\right)$

(ii) If $X$ is locally isomorphic with $\operatorname{Spec} C\left[x_{1}, x_{2}, x_{3}, z\right] /\left(x_{1} x_{2} x_{3}\right)$ along $Z=\operatorname{Spec} C[z]$ then we connect all the vertices at one point where $D_{i}=$ $\operatorname{Spec} C\left[x_{i}, z\right]$.

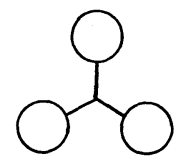

Otherwise we don't connect vertices any more. Notice that a graph thus obtained is not necessarily connected. Under the assumption (A), any irreducible subcheme of codimension one in $X$ is reduced, so the above definition of a graph seems to be natural. However the author does not know how we should define it in the general case. Anyway in the three dimensional case the condition (A) does hold for any $\Sigma$. We shall give here a complete list of graphs of $\mathrm{D}-\mathrm{V}$ cones in $\overline{\mathfrak{Y}}_{3}^{+}$. In the following table an arrow starting from a vertex into itself means that
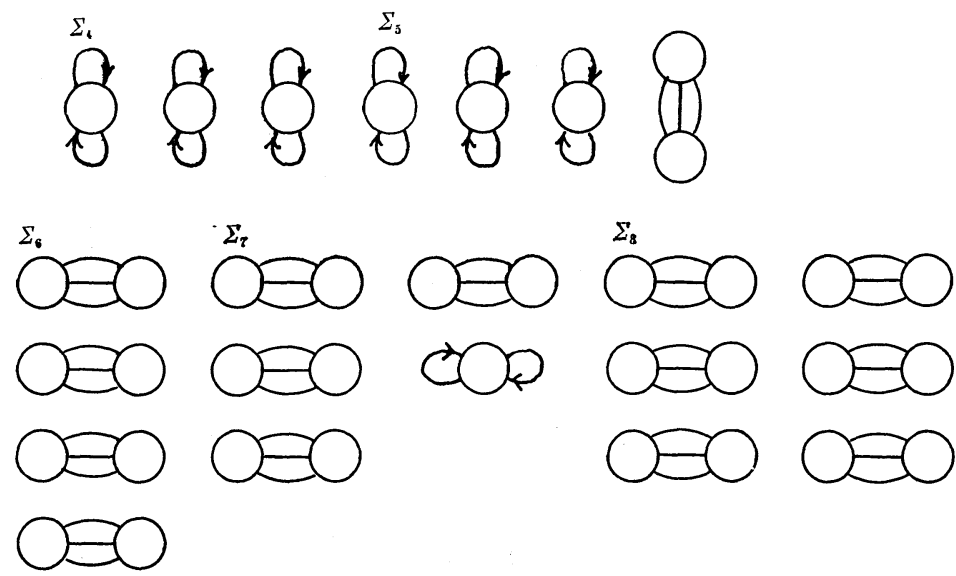
a subscheme corresponding to the vertex intersects with itself along a one-codimensional subscheme of it. Three arrows from one vertex to another imply that two subschemes of codimension one corresponding to vertices meet along three distinct subschemes of codimension two.

In order to make our idea clear, we shall show that in case of $\Sigma_{5}$, the associated graph is given as above. As stated before the Delone decomposition consists of two trigular prisms and their faces modulo

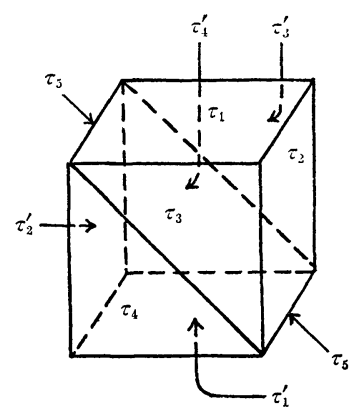

translation. We call the upper face $\tau_{1}$, the lower $\tau_{1}^{\prime}$ respectively. Also we call the face on the right hand $\tau_{2}$ and its reverse face $\tau_{2}^{\prime}$. Moreover we call the faces on this side, $\tau_{3}$ and $\tau_{4}$ from above, and their counterparts $\tau_{3}^{\prime}, \tau_{4}^{\prime}$ respectively. $\tau_{i}$ coincide with $\tau_{i}^{\prime}$ modulo translation by an integral vector. We call the face contained in the interior of the cube $\tau_{5}$. On the other hand any pair of oppositive lines on $\tau_{1}$ (or $\tau_{2}, \tau_{5}$ ) are coincident with each other. Thus we have the former three graphs.
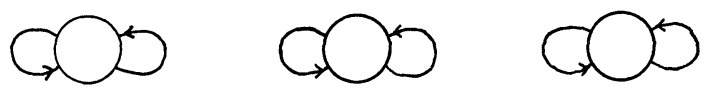

And also the upper edge of $\tau_{3}$ and the lower edge of $\tau_{4}$ are coincident, and so are the right edge of $\tau_{3}$ and the left of $\tau_{4}$. Hence we obtain the last graph.

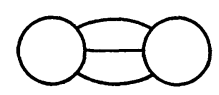

(5.4) In order to compute $\operatorname{Ext}^{1}\left(\Omega_{X}^{1} \mathcal{O}_{X}\right)$ for a SQAV $X$ whose corresponding $\mathrm{D}-\mathrm{V}$ cone is one of $\Sigma_{i}(4 \leqq i \leqq 8)$ we require the following lemmae. 
LEMma 5.1. Suppose $X$ to be a Cohen-Macaulay scheme of dimension $n(n \geqq 3)$, and $Z$ a subscheme of $X$ of at least three codimension. Let $i$ be an inclusion of $X-Z$ in $X$. Then we have, $\mathscr{E} x t^{q}\left(\Omega_{X}^{1} \mathcal{O}_{X}\right)=$ $i_{*} \mathscr{E}_{x t^{q}}\left(\Omega_{X-Z}^{1}, \mathcal{O}_{X-Z}\right) . \quad(q=0,1)$

Proof. We have a usual exact sequence of local cohomology,

$$
\begin{aligned}
0 & \rightarrow \mathscr{E} x t^{0}{ }_{Z}\left(\Omega_{X}^{1} \mathcal{O}_{X}\right) \rightarrow \mathscr{E} x t^{0}\left(\Omega_{X}^{1} \mathcal{O}_{X}\right) \rightarrow i_{*} \mathscr{E}_{x t^{0}}\left(\Omega_{X-Z}^{1} \mathcal{O}_{X-Z}\right) \\
& \rightarrow \mathscr{E} x t_{Z}{ }_{Z}\left(\Omega_{X}^{1} \mathcal{O}_{X}\right) \rightarrow \mathscr{E} x t^{1}\left(\Omega_{X}^{1} \mathcal{O}_{X}\right) \rightarrow i_{*} \mathscr{E}_{x t}{ }^{1}\left(\Omega_{X-Z}^{1} \mathcal{O}_{X-Z}\right) \\
& \rightarrow \mathscr{E} x t^{2}{ }_{Z}\left(\Omega_{X}^{1} \mathcal{O}_{X}\right) \rightarrow \cdots
\end{aligned}
$$

On the other hand there is a spectral sequence

$$
E_{2}^{p q}=\mathscr{E}_{x t}^{p}\left(\Omega_{X}^{1} \mathscr{H}_{Z}^{q}\left(\mathcal{O}_{X}\right)\right) \text { converging to } \mathscr{E}_{x t_{Z}^{p+q}}\left(\Omega_{X}^{1} \mathcal{O}_{X}\right) \text {. }
$$

By assumption we have $\mathscr{H}_{Z}^{q}\left(\mathcal{O}_{X}\right)=0(q=0,1,2)$, so that our lemma follows.

Q.E.D.

To a connected component $\Delta$ of the graph of $X$ we associate a connected reduced subvariety $Z_{\Delta}$ of codimension 1 in $X$ whose support is a union of $X_{\tau}$, where $X_{\tau}$ denotes the divisor corresponding to a Delone cell $\tau(\subset \Delta)$.

Our main interest is to know the number of connected components of the graph of $X$ because of the following lemma e.

LEMMA 5.2. Under the assumption $\left(A^{*}\right), \mathscr{E}_{x t^{1}}\left(\Omega_{X}^{1} \mathcal{O}_{X}\right)=i_{*}\left(\oplus_{\Delta} F_{\Delta}\right)$ where $\Delta$ runs over the set of connected components of the graph of $X$ and $F_{\Delta}$ is an invertible sheaf on $Z_{\Delta}-Z$.

Proof of Lemma 5.2. Since the condition (A) is satisfied in view of Lemma 1.6., the structure of $X$ is given in proposition 2.3.. Let $Z=\cup_{r} Z_{\gamma}$ be a union of all the subvarieties $Z_{\gamma}(\gamma:$ Delone $(g-3)$ cell $)$. According to Lemma 5.1 we suffice to investigate $\mathscr{E}_{x t^{1}}\left(\Omega_{X-Z}^{1}, \mathcal{O}_{X-Z}\right)$. Let $X_{k_{\rho}}=\operatorname{Spec} C\left[x_{a}^{(k)}, a \in \Phi,\left(x_{a}^{(k)}\right)^{-1}, a+k \in \rho\right]$ be an open subscheme of $\left(U_{k}\right)_{0}$. $Z_{\Delta}-Z$ is covered with the open affine sets $X_{k, \rho}$.

Since $\left(U_{k}\right)_{0} \cong\left(U_{0}\right)_{0}$ we shall prove $\mathscr{E} x t^{1}\left(\Omega_{X_{0 \rho} \rho}^{1} \mathcal{O}_{X_{0 \rho}}\right)=\oplus \mathcal{O}_{Z_{\Delta} \cap X_{0 \rho}}$ on $X_{0 \rho}$.

In what follows, we denote Delone cells containing the origin by $\sigma$, $\tau, \rho$.

Let $\sigma$ be a Delone $g$-cell, $\sigma=\left\{x \in \boldsymbol{R}^{g} ; c_{i}{ }^{t} x \geqq 0 i=1, \cdots, N\right\}, \rho$ be a Delone $(g-2)$ cell defined by $\rho=\left\{x \in \sigma ; c_{i}{ }^{t} x=c_{2}{ }^{t} x=0\right\}$. Then $\tau_{\nu}=$ $\left\{x \in \sigma ; c_{\nu}{ }^{t} x=0\right\}$ is a face of $\sigma$, and $\rho$ is a face of $\tau_{\nu}$.

By assumption $\left(A^{*}\right)$, there exists Delone 1-cell $\hat{a}_{\nu}(\nu=1,2)$ such that $Z_{0}^{+} \hat{a}_{\nu}+Z_{0}^{+} \rho=\left\{n_{1} a_{\nu}+\Sigma m_{j} a_{j} ; a_{j} \in \rho n_{1} \geqq 0, m_{j} \geqq 0\right\}$ contains a basis of $Z \tau_{\nu}=$ 
$\left\{\sum m_{j} a_{j}, a_{j} \in \tau_{\nu}, m_{j} \in Z\right\}$. Hence, any vertex a of $\sigma$ is by assumption $\left(A^{*}\right)$ written as a linear combination of $\hat{a}_{1}, \hat{a}_{2}$ and vertices of $\rho$. Let $a=$ $n_{1} \hat{a}_{1}+n_{2} \hat{a}_{2}+\sum_{j} m_{j} a_{j}, a_{j} \in \rho . \quad$ Since $c_{t}^{1} a=c_{t}^{1}\left(n_{1} \hat{a}_{1}+n_{2} \hat{a}_{2}\right)=n_{2} c_{1}^{t} \hat{a}_{2} \geq 0, c_{1}^{t} \hat{a}_{2}>0$, we have $n_{2} \geq 0$. Similarly $n_{1} \geq 0$. Then we have $x_{a}=x_{\hat{a}_{1}}^{n_{1}} \cdot x_{\hat{a}_{2}}^{n_{2}} \cdot \prod x_{a_{j}}^{m_{j}}$, i.e., $x_{a}$ is contained in $C\left[x_{\hat{a}_{1}}, x_{\hat{a}_{2}}, x_{a}^{ \pm 1}, a \in \rho\right]$. Hence, $X_{0_{\rho}} \cap Z_{\sigma} \cong \operatorname{Spec} C\left[x_{\hat{a}_{1}}, x_{\hat{a}_{2}}\right.$, $\left.x_{a}^{ \pm 1}, a \in \rho\right]$. Therefore $X_{0 \rho}$ is isomorphic with $Y_{n} \times \operatorname{Spec} C\left[x_{a}^{ \pm 1}, a \in \rho\right]$ where $Y_{n}$ is one of the affine schemes given in (4.1.). According to Lemma 4.1., we have an isomorphism $\mathscr{E}_{x t^{1}}\left(\Omega_{X_{0 \rho}}^{1}, \mathcal{O}_{X_{0 \rho}}\right) \cong \oplus \mathcal{O}_{\Sigma_{\Delta} \cap X_{0 \rho}}$.

LEMMA 5.3. If $g=3$, then $F_{\Delta}=\mathcal{O}_{Z_{\Delta}-Z}$, a fortiori $\mathscr{E}_{x t^{1}}\left(\Omega_{X}^{1} \mathcal{O}_{X}\right) \cong$ $\oplus \mathcal{O}_{Z_{\Delta}}$. In particular $\operatorname{dim} H^{0}\left(X, \mathscr{E} x t^{1}\left(\Omega_{X}^{1} \mathcal{O}_{X}\right)\right)=\#($ connected components of the graph of $X$ ).

Proof. Since $F_{\Delta}$ is invertible on $Z_{\Delta}-Z$, we suffices to show the existence of a global section of $\mathscr{E}_{x t^{1}}\left(\Omega_{X}^{1} \mathcal{O}_{X}\right)$ vanishing nowhere on $Z_{\Delta}-Z$. In fact, $Z_{\Delta}$ is Cohen-Macaulay so that $i_{*} \mathcal{O}_{Z_{\Delta-Z}}=\mathcal{O}_{Z_{\Delta}}$. The existence of a non vanishing section is proved by investigating closely a local family of deformations of $X$. The proof is a case by case examination. We deal with only the case $\Sigma=\Sigma_{6}$ for brevity. With the notations in (5.5), (5.6) we put

$$
\begin{gathered}
\tilde{\tau}(s)=\left(\begin{array}{ccc}
0 & 0 & \tau_{13} \\
0 & 0 & \tau_{23} \\
\tau_{13} & \tau_{23} & 0
\end{array}\right)+e_{1} \log s_{11} / 2 \pi \sqrt{-1}+e_{12} \log s_{12} / 2 \pi \sqrt{-1} \\
\quad+e_{23} \log s_{22} / 2 \pi \sqrt{-1}+e_{3} \log s_{33} / 2 \pi \sqrt{-1} \\
2 r(s)=\left(\log \left(s_{11} s_{12}\right) 2 \pi \sqrt{-1}, \quad \log \left(s_{12} s_{22}\right) 2 \pi \sqrt{-1}, \quad \log \left(s_{22} s_{23}\right) / 2 \pi \sqrt{-1}\right) \\
\tilde{R}(\tilde{\tau})=\mathcal{O}\left[\tilde{\xi}_{m} \cdot \theta, m \in Z^{3}\right], \quad \mathcal{O}=\mathcal{O}_{0 E}, \quad E=\left\{\left(s_{i j}\right) \in C^{4} ;\left|s_{i j}\right|<\varepsilon\right\} \\
\left.\tilde{P}=\operatorname{Proj} \tilde{R}(\tilde{\tau}), \quad X=\tilde{P} / \Gamma, \quad \Gamma=Z^{3} . \quad \text { (see }(2.4)\right)
\end{gathered}
$$

Then $\tilde{P}$ is covered with $U_{k}, k \in Z^{3} . \quad U_{0}$ is given by,

$$
\operatorname{Spec} \mathcal{O}\left(\begin{array}{l}
w_{i}^{-1}(i=1,2,3), s_{11} s_{12} w_{1}, s_{12} s_{22} w_{2}, s_{22} s_{33} w_{3} \\
s_{12}^{-1} w_{1}^{-1} w_{2}^{-1}, s_{22}^{-1} w_{2}^{-1} w_{3}^{-1}, s_{11} s_{12} s_{22} w_{1} w_{2}, s_{12} s_{22} s_{33} w_{2} w_{3} \\
s_{12}^{-1} s_{22}^{-1} w_{1}^{-1} w_{2}^{-1} w_{3}^{-1}, s_{11} s_{12} s_{22} s_{33} w_{1} w_{2} w_{3}
\end{array}\right)
$$

We shall show $\partial / \partial s_{33}$ is mapped by the Kodaira-Spencer mapping into a section vanishing nowhere on $Z_{\Delta_{1}}-Z, \Delta_{1}=\tau_{1} \cup \tau_{2}$.

At generic points of $Z_{\tau_{1}}$ and $Z_{\tau_{2}}$, we have

$$
\begin{array}{lll}
U_{0} \cong \operatorname{Spec} \mathcal{O}\left[x_{a_{1}}^{ \pm 1}, x_{a_{4}}^{ \pm 1}, x_{a_{6}}, w_{3}^{-1}\right], & \frac{x_{a_{6}} w_{3}^{-1}=s_{33} x_{a_{4}}}{x_{0}} & \text { along } Z_{\tau_{1}} \\
U_{0} \cong \operatorname{Spec} \mathcal{O}\left[x_{a_{2}}^{ \pm 1}, x_{a_{4}}^{ \pm 1}, x_{a_{5}}, w_{3}^{-1}\right], & \underline{x_{a_{5}} w_{3}^{-1}=s_{33} x_{a_{2}}} & \text { along } Z_{\tau_{2}}
\end{array}
$$


Moreover at generic points of $Z_{a_{1}}, Z_{a_{2}}$ and $Z_{a_{4}}$ respectively we have

$$
\begin{aligned}
U_{0} \cong & \operatorname{Spec} \mathcal{O}\left[x_{a_{1}}^{ \pm 1}, x_{a_{4}}, x_{a_{6}}, w_{2}^{-1}, s_{22}^{-1} w_{2}^{-1} w_{3}^{-1}\right] \\
& x_{a_{4}} \cdot w_{2}^{-1}=s_{22} x_{a_{1}}, x_{a_{6}} \cdot s_{22}^{-1} w_{2}^{-1} w_{3}^{-1}=s_{33} x_{a_{1}} \\
U_{0} \cong & \operatorname{Spec} \mathcal{O}\left[x_{a_{2}}^{ \pm 1}, x_{a_{4}}, x_{a_{5}}, w_{1}^{-1}, w_{3}^{-1}\right] \\
& x_{a_{4}} \cdot w_{1}^{-1}=s_{11} x_{a_{2}}, x_{a_{5}} \cdot w_{3}^{-1}=s_{33} x_{a_{2}} \\
U_{0} \cong & \operatorname{Spec} \mathcal{O}\left[x_{a_{4}}^{ \pm 1}, x_{a_{1}}, x_{a_{6}}, x_{a_{2}}, w_{3}^{-1}\right] \\
& x_{a_{1}} x_{a_{2}}=s_{12} x_{a_{4}}, x_{a_{6}} \cdot w_{3}^{-1}=s_{33} x_{a_{4}}
\end{aligned}
$$

Therefore the image of $\partial / \partial s_{33}$ vanishes nowhere on $Z_{\Delta_{1}}-Z$. By the same way as above, we can show the existence of a non vanishing section for any $\Delta$, which completes the proof.

(Q.E.D.)

In view of Lemma 5.3. we obtain,

COROLlary OF LEMma 5.3.

$$
\operatorname{dim} H^{0}\left(X, \mathscr{E}_{x t^{1}}\left(\Omega_{X}^{1} \mathcal{O}_{X}\right)\right)= \begin{cases}3 & \left(\Sigma=\Sigma_{4}\right) \\ 4 & \left(\Sigma=\Sigma_{5}, \Sigma_{6}\right) \\ 5 & \left(\Sigma=\Sigma_{7}\right) \\ 6 & \left(\Sigma=\Sigma_{8}\right)\end{cases}
$$

(5.5) As a special case of proposition 2.5. we have

LEMMA 5.4. With the notations in (4.1.) the following sequence is exact $(n \geqq 3)$,

$$
0 \longrightarrow \mathscr{E} x t^{0}\left(\Omega_{Y}^{1} \mathcal{O}_{Y}\right) \stackrel{\iota}{\longrightarrow} \mathcal{O}_{\tilde{Y}}^{+2} \stackrel{\psi_{0}}{\longrightarrow} \bigoplus_{i=1}^{n} \mathcal{O}_{D_{i}} \longrightarrow 0
$$

where $\tilde{Y}$ denotes the normalization of $Y$ and $D_{i}=\operatorname{Spec} C\left[x_{1}, \cdots, x_{n}\right] /\left(x_{1}\right.$, $\left.\cdots, x_{i-1}, x_{i+1}, \cdots, x_{n}\right)$. The proof is straightforward.

Proposition 5.1. Under the assumption $\left(A^{*}\right)$ we have an exact sequence,

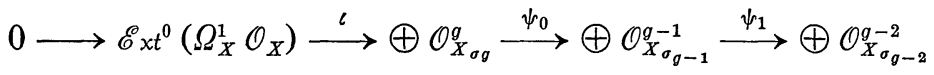

$$
\begin{aligned}
& \text { on } X-Z
\end{aligned}
$$

where $Z=\cup_{r} Z_{\gamma}(\gamma:$ Delone $(g-3)$ cell $), \psi_{k}\left(\left(f_{a_{\sigma}}\right)_{a \subset \sigma}\right)=\left(\sum_{\tau \prec \sigma}[\sigma: \tau] f_{a_{\tau}}\right)_{a \subset \tau}$ and the other notations are the same as in proposition 2.5.

Proof. As seen in the proof of lemma 5.2., $X-Z$ is covered with $X_{k, \rho} . \quad X_{k \rho}=\operatorname{Spec} C\left[\xi_{a}^{(k)}, a \in \Phi,\left(\xi_{a}^{(k)}\right)^{-1}, a+k \in \rho\right] \cong Y_{n} \times \operatorname{Spec} C\left[x_{a}^{ \pm 1}, a \in \rho\right]$ for 
some $n$. Hence according to lemma 5.4., we have

$$
\mathscr{E} x t^{0}\left(\Omega_{X}^{1} \mathcal{O}_{X}\right)=\mathscr{E}_{x t} t^{0}\left(\Omega_{Y}^{1} \mathcal{O}_{Y}\right) \otimes \mathcal{O}_{X} \oplus \mathcal{O}_{X}^{g-2}
$$

so that we obtain the above exact sequence.

Remark. We notice the sequence $(* *)$ is defined on the whole $X$. The author conjectures (**) is exact on $X$.

LEMMA 5.5. Consider a sequence of $\mathcal{O}_{X}$ modules

$$
0 \longrightarrow F \stackrel{\iota}{\longrightarrow} A_{0} \stackrel{\psi_{0}}{\longrightarrow} A_{1} \stackrel{\psi_{1}}{\longrightarrow} A_{2}
$$

such that $\psi_{1} \circ \psi_{0}=0, \psi_{0} \circ \iota=0$. Assume $0 \rightarrow F \rightarrow A_{0} \rightarrow A_{1}$ to be exact, $H^{1}\left(X, A_{0}\right)=0$. Then we have $\operatorname{dim} H^{1}(X, F) \leqq \operatorname{dim} H^{1}\left(\left\{H^{0}\left(X, A_{j}\right)\right\}\right)$.

Proof. Let $B$ be the image of $A_{0}$. Then we have a long exact sequence

$$
0 \rightarrow H^{0}(X, F) \rightarrow H^{0}\left(X, A_{0}\right) \rightarrow H^{0}(X, B) \rightarrow H^{1}(X, F) \rightarrow 0 .
$$

Hence

$$
\begin{aligned}
\operatorname{dim} H^{1}(X, F) & =\operatorname{dim} H^{0}(X, B) / \psi_{0}\left(H^{0}\left(X, A_{0}\right)\right. \\
& \leqq \operatorname{dim} \operatorname{Ker}\left(H^{0}\left(A_{1}\right) \rightarrow H^{0}\left(A_{2}\right)\right) / \psi_{0}\left(H^{0}\left(A_{0}\right)\right) .
\end{aligned}
$$

Now we shall compute the upper bound of $\operatorname{dim} H^{1}\left(X, \operatorname{Ext}^{0}\left(\Omega^{1}{ }_{X} \mathcal{O}_{X}\right)\right)$ using the above lemma 5.5. Since each irreducible component $X_{\sigma}$ is rational, so $H^{i}\left(X_{\sigma} \mathcal{O}_{X_{\sigma}}\right)$ vanishes for $i>0$ and any Delone cell $\sigma$ ([11] p. 44, 52) $\operatorname{dim} H^{1}\left(X, \operatorname{Ext}^{0}\left(\Omega_{X}^{1} \mathcal{O}_{X}\right)\right)$ is dominated by the dimension of the cohomology of the complex,

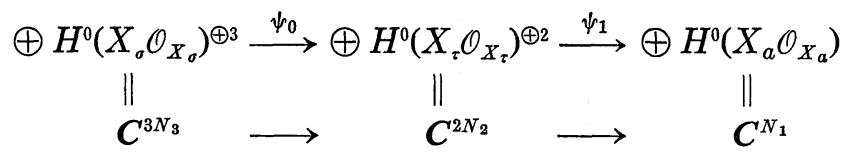

where $N_{\nu}$ denotes the number of $\nu$-dimensional Delone cells $\bmod \Gamma$. In this case $N_{1}=6, N_{2}=8, N_{3}=3$.

We define oriented cells $\sigma_{i}, \tau_{j}, a_{k} \bmod \Gamma$ as follows

$$
\begin{aligned}
& \sigma_{1}=\left\langle P_{1}, P_{5}, P_{6}, P_{7}, P_{8}\right\rangle \cup\left\langle P_{7}, P_{1}, P_{2}, P_{3}, P_{4}\right\rangle \\
& \sigma_{2}=\left\langle P_{1}, P_{2}, P_{6}, P_{7}\right\rangle \\
& \sigma_{3}=\left\langle P_{1}, P_{4}, P_{7}, P_{8}\right\rangle \\
& \tau_{1}=\left\langle P_{1}, P_{2}, P_{6}\right\rangle
\end{aligned}
$$




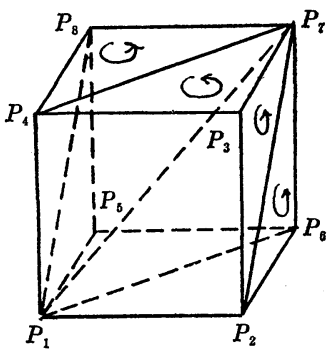

$$
\begin{aligned}
& \tau_{2}=\left\langle P_{1}, P_{6}, P_{5}\right\rangle \\
& \tau_{3}=\left\langle P_{1}, P_{5}, P_{8}\right\rangle \\
& \tau_{4}=\left\langle P_{1}, P_{8}, P_{4}\right\rangle \\
& \tau_{5}=\left\langle P_{1}, P_{2}, P_{7}\right\rangle \\
& \tau_{6}=\left\langle P_{1}, P_{7}, P_{8}\right\rangle \\
& \tau_{7}=\left\langle P_{1}, P_{6}, P_{7}\right\rangle \\
& \tau_{8}=\left\langle P_{1}, P_{7}, P_{4}\right\rangle \\
& a_{1}=\left\langle P_{1}, P_{2}\right\rangle, \quad a_{2}=\left\langle P_{1}, P_{5}\right\rangle, \quad a_{3}=\left\langle P_{1}, P_{4}\right\rangle \\
& a_{4}=\left\langle P_{1}, P_{6}\right\rangle, \quad a_{5}=\left\langle P_{1}, P_{8}\right\rangle, \quad a_{6}=\left\langle P_{1}, P_{7}\right\rangle
\end{aligned}
$$

where $P_{i}$ denotes a point in $\boldsymbol{R}^{3}$ whose coordinate is given by,

$$
\begin{array}{lll}
P_{1}=(0,0,0), & P_{2}=(1,0,0), & P_{3}=(1,0,1) \\
P_{4}=(0,0,1), & P_{5}=(0,1,0), & P_{6}=(1,1,0) \\
P_{7}=(1,1,1), & P_{8}=(0,1,1) . &
\end{array}
$$

Then the homomorphisms $\psi_{0}, \psi_{1}$ are given by,

$$
\begin{aligned}
& \psi_{0}\left(\left(f_{a_{\sigma}}\right)_{a \subset \sigma}\right)=\left(\sum_{\tau \prec \sigma}[\sigma: \tau] f_{a_{\sigma}}\right)_{\tau} \\
& \psi_{1}\left(\left(f_{a_{\tau}}\right)_{a \subset \tau}\right)=\left(\sum_{a \prec \tau}[\tau: a] f_{a_{\tau}}\right)_{a} .
\end{aligned}
$$

More explicitly, with the notations as above $\psi_{1}$ is given by,

$$
\begin{aligned}
& {\left[\tau_{1}: a_{1}\right] f_{a_{1 \tau_{1}}}+\left[\tau_{2}: a_{1}\right] f_{a_{1 \tau_{2}}}+\left[\tau_{5}: a_{1}\right] f_{a_{1 \tau_{5}}}+\left[\tau_{6}: a_{1}\right] f_{a_{1 \tau_{6}}},} \\
& {\left[\tau_{1}: a_{2}\right] f_{a_{2 \tau_{1}}}+\left[\tau_{2}: a_{2}\right] f_{a_{2 \tau_{2}}}+\left[\tau_{3}: a_{2}\right] f_{a_{2 \tau_{3}}}+\left[\tau_{4}: a_{2}\right] f_{a_{2 \tau_{4}}},} \\
& {\left[\tau_{3}: a_{3}\right] f_{a_{3 \tau_{3}}}+\left[\tau_{4}: a_{3}\right] f_{a_{3} \tau_{4}}+\left[\tau_{7}: a_{3}\right] f_{a_{3} \tau_{7}}+\left[\tau_{8}: a_{3}\right] f_{a_{3 \tau_{8}}},} \\
& {\left[\tau_{1}: a_{4}\right] f_{a_{4 \tau_{1}}}+\left[\tau_{2}: a_{4}\right] f_{a_{4} \tau_{2}}+\left[\tau_{7}: a_{4}\right] f_{a_{4} \tau_{7}}+\left[\tau_{8}: a_{4}\right] f_{a_{4} \tau_{8}},} \\
& {\left[\tau_{3}: a_{5}\right] f_{a_{5} \tau_{3}}+\left[\tau_{4}: a_{5}\right] f_{a_{5} \tau_{4}}+\left[\tau_{5}: a_{5}\right] f_{a_{5} \tau_{5}}+\left[\tau_{6}: a_{5}\right] f_{a_{5} \tau_{6}},} \\
& {\left[\tau_{5}: a_{6}\right] f_{a_{6 \tau_{5}}}+\left[\tau_{6}: a_{6}\right] f_{a_{6} \tau_{6}}+\left[\tau_{7}: a_{6}\right] f_{a_{6} \tau_{7}}+\left[\tau_{8}: a_{6}\right] f_{a_{6} \tau_{8}},}
\end{aligned}
$$


Notice that

$$
\begin{array}{ll}
f_{a_{4} \tau_{1}}=f_{a_{1 \tau_{1}}}+f_{a_{2 \tau_{1}}}, & f_{a_{4} \tau_{2}}=f_{a_{1 \tau_{2}}}+f_{a_{2 \tau_{2}}}, \\
f_{a_{5} \tau_{3}}=f_{a_{2} \tau_{3}}+f_{a_{3 \tau_{3}}}, & f_{a_{5} \tau_{4}}=f_{a_{2 \tau_{4}}}+f_{a_{3} \tau_{4}}, \\
f_{a_{6} \tau_{5}}=f_{a_{1 \tau_{5}}}+f_{a_{5} \tau_{5}}, & f_{a_{6} \tau_{6}}=f_{a_{1 \tau_{6}}}+f_{a_{5} \tau_{8}}, \\
f_{a_{6} \tau_{7}}=f_{a_{3} \tau_{7}}+f_{a_{4} \tau_{7}}, & f_{a_{6 \tau_{8}}}=f_{a_{3} \tau_{8}}+f_{a_{4} \tau_{8}} .
\end{array}
$$

Since $\sum_{a \prec \tau}[\tau: a] f_{a, \tau}=0$ where the summation is all over the pairs $a \prec \tau$, it is easy to check $\operatorname{dim} \operatorname{Im} \psi_{1}=5$. Similary we have $\operatorname{dim} \operatorname{Im} \psi^{0}=6$, so $\operatorname{dim} H^{1}\left(X, \mathscr{E}_{x t^{0}}\left(\Omega_{X}^{1} \mathcal{O}_{X}\right)\right) \leqq 5$,

In the same way as above we obtain

LEMMA 5.4.

$$
\operatorname{dim} H^{1}\left(X, \mathscr{E} x t^{0}\left(\Omega_{X}^{1} \mathcal{O}_{X}\right)\right) \leqq \begin{cases}6 & \left(\Sigma=\Sigma_{4}\right) \\ 5 & \left(\Sigma=\Sigma_{5}, \Sigma_{6}\right) \\ 4 & \left(\Sigma=\Sigma_{7}\right) \\ 3 & \left(\Sigma=\Sigma_{8}\right)\end{cases}
$$

According to Corollary of Lemma 5.3, Lemma 5.6, we have $\operatorname{dim} \operatorname{Ext}^{1}\left(\Omega_{X}^{1} \mathcal{O}_{X}\right) \leqq 9$ for a $\operatorname{SQAV} X$ of level 1 .

(5.6) Next we shall construct a flat family of deformations of $X$ depending on 9 effective parameters.

Because of the similarity of computations we deal with only the case $\Sigma=\Sigma_{6}$ as a succession of (5.5). Let $X$ be a SQAV whose corresponding $\mathrm{D}-\mathrm{V}$ cone is $\Sigma_{6}$. Let

$$
\tau(s)=\left(\begin{array}{ccc}
0 & 0 & \tau_{13} \\
0 & 0 & \tau_{23} \\
\tau_{13} & \tau_{33} & 0
\end{array}\right)+\left(\begin{array}{rrr}
2 & -1 & 0 \\
-1 & 2 & -1 \\
0 & -1 & 2
\end{array}\right) \log s / 2 \pi \sqrt{-1}
$$

( $\tau_{i j}$ : constant). Then $X$ is the closed fiber of $\operatorname{Proj} R(\tau) / \Gamma$.

We put

$$
\begin{aligned}
\tilde{\tau}(s)= & \left(\begin{array}{ccc}
0 & 0 & \tau_{13}+s_{13} \\
s_{21} & 0 & \tau_{23}+s_{23} \\
\tau_{13}+s_{31} & \tau_{23}+s_{32} & 0
\end{array}\right)+\left(\begin{array}{lll}
1 & 0 & 0 \\
0 & 0 & 0 \\
0 & 0 & 0
\end{array}\right) \log s_{11} / 2 \pi \sqrt{-1} \\
& +\left(\begin{array}{rrr}
1 & -1 & 0 \\
-1 & 1 & 0 \\
0 & 0 & 0
\end{array}\right) \log s_{12} / 2 \pi \sqrt{-1}+\left(\begin{array}{rrr}
0 & 0 & 0 \\
0 & 1 & -1 \\
0 & -1 & 1
\end{array}\right) \log s_{22} / 2 \pi \sqrt{-1} \\
& +\left(\begin{array}{lll}
0 & 0 & 0 \\
0 & 0 & 0 \\
0 & 0 & 1
\end{array}\right) \log s_{33} / 2 \pi \sqrt{-1}
\end{aligned}
$$




$$
\begin{gathered}
2 r(s)=\left(\log \left(s_{11} s_{12}\right) / 2 \pi \sqrt{-1}, \quad \log \left(s_{12} s_{22}\right) / 2 \pi \sqrt{-1}, \quad \log \left(s_{22} s_{33}\right) / 2 \pi \sqrt{-1}\right), \\
\tilde{R}(\tilde{\tau})=\mathcal{O}\left[\tilde{\xi}_{m} \cdot \theta, m \in Z^{3}\right], \quad \mathcal{O}=\mathcal{O}_{0, E}, \quad E=\left\{\left(s_{i j}\right) \in C^{9} ;\left|s_{i j}\right|<\varepsilon\right\} \\
\tilde{\xi}_{m}=e\left(\frac{1}{2} m \tilde{\tau}(s)^{t} m+m^{t}(\zeta+r(s))\right), \quad x_{a}=\tilde{\xi}_{a} \\
\mathscr{X}=\operatorname{Proj} \tilde{R}(\tilde{\tau}) / \Gamma, \quad \Gamma=Z^{3} . \quad \text { (see (2.4.) Remark) }
\end{gathered}
$$

Then $\mathscr{X}$ is covered with affine open sets $U_{k}\left(k \in Z^{3}\right), U_{k}=\operatorname{Spec} \mathcal{O}\left[\tilde{\xi}_{m} \tilde{\xi}_{k}^{-1}\right.$, $m \in Z^{3}$. More explicitly $U_{0}$ is given by,

$$
U_{0}=\operatorname{Spec} \mathcal{O}\left(\begin{array}{l}
w_{i}^{-1}(i=1,2,3), s_{11} s_{12} w_{1}, s_{12} s_{22} w_{2}, s_{22} s_{33} w_{3} \\
s_{12}^{-1} w_{1}^{-1} w_{2}^{-1}, s_{22}^{-1} w_{2}^{-1} w_{3}^{-1}, s_{11} s_{12} s_{22} w_{1} w_{2}, s_{12} s_{22} s_{33} w_{2} w_{3} \\
s_{12}^{-1} s_{22}^{-1} w_{1}^{-1} w_{2}^{-1} w_{3}^{-1}, s_{11} s_{12} s_{22} s_{33} w_{1} w_{2} w_{3}
\end{array}\right)
$$

It is easy to check $\mathscr{X}$ is Cohen-Macaulay. ([11]) Since the fibering of $\mathscr{X}$ over $\operatorname{Spec} \mathscr{O}$ is equidimensional and $\operatorname{Spec} \mathcal{O}$ is smooth, the projection $\varpi$ from $\mathscr{X}$ to $\operatorname{Spec} \mathcal{O}$ is flat. Obviously the fiber $\widetilde{\varpi}^{-1}(0)$ is $X$. In order to show the Kodaira-Spencer mapping from $T_{0}(E)$ to $\operatorname{Ext}^{1}\left(\Omega_{X}^{1} \mathcal{O}_{X}\right)$ is surjective, we shall begin with showing that $H^{0}\left(X, \mathscr{E} x t^{1}\left(\Omega_{X}^{1} \mathcal{O}_{X}\right)\right)$ is spanned by $\partial / \partial s_{11}, \partial / \partial s_{12}, \partial / \partial s_{22}, \partial / \partial s_{33}$. We use the notations in (5.5). Denote four connected components of the graph of $X$ by $\Delta_{1}=\tau_{1} \cup \tau_{2}, \Delta_{2}=\tau_{3} \cup \tau_{4}$, $\Delta_{3}=\tau_{5} \cup \tau_{6}, \Delta_{4}=\tau_{7} \cup \tau_{8}$. Let $P_{1}$ be a generic point of $Z_{\tau_{1}} \cap U_{0}$. At $P_{1}$, we have $x_{a_{1}} \neq 0, x_{a_{4}} \neq 0$, so that $U_{0}$ near $P_{1}$ is isomorphic with $\operatorname{Spec} \mathcal{O}\left[x_{a_{1}}^{ \pm 1}\right.$, $\left.x_{a_{4}}^{ \pm 1}, x_{a_{6}}, w_{3}^{-1}\right]$. We notice that $x_{a_{6}} \cdot w_{3}^{-1}=s_{33} x_{a_{4}}$. Hence $\partial / \partial s_{33}$ is projected by Kodaira-Spencer mapping to a basis of $H^{0}\left(Z_{\Delta_{1}} \mathcal{O}_{Z_{\Delta_{1}}}\right)\left(\subset H^{0}\left(X, \mathscr{E} x t^{1}\left(\Omega_{X}^{1} \mathcal{O}_{X}\right)\right)\right.$. Next let $P_{2}, P_{3}$ and $P_{4}$ be generic points of $Z_{\tau_{3}} \cap U_{0}, Z_{\tau_{5}} \cap U_{0}, Z_{\tau_{7}} \cap U_{0}$ respectively. $U_{0}$ near $P_{i}$ is isomorphic with

$$
\begin{array}{ll}
\operatorname{Spec} \mathcal{O}\left[x_{a_{2}}^{ \pm 1}, x_{a_{5}}^{ \pm 1}, x_{a_{4}}, w_{1}^{-1}\right], x_{a_{4}} \cdot w_{1}^{-1}=s_{11} x_{a_{2}} & (i=2) \\
\operatorname{Spec} \mathcal{O}\left[x_{a_{1}}^{ \pm 1}, x_{a_{6}}^{ \pm 1}, x_{a_{3}}, x_{a_{4}}\right], x_{a_{3}} x_{a_{4}}=s_{22} x_{a_{6}} & (i=3) \\
\operatorname{Spec} \mathcal{O}\left[x_{a_{4}}^{ \pm 1}, x_{a_{6}}^{ \pm 1}, x_{a_{1}}, x_{a_{2}}\right], x_{a_{1}} x_{a_{2}}=s_{12} x_{a_{4}} & (i=4)
\end{array}
$$

Hence $\partial / \partial s_{11}, \partial / \partial s_{22}, \partial / \partial s_{12}$ are projected to a basis of $H^{0}\left(Z_{\Delta_{2}}, \mathcal{O}_{Z_{\Delta_{2}}}\right), H^{0}\left(Z_{\Delta_{3}}, \mathcal{O}_{Z_{\Delta_{3}}}\right)$, $H^{0}\left(Z_{\Delta_{4}}, \mathcal{O}_{Z_{\Delta_{4}}}\right)$ respectively.

It remains to show $\partial / \partial s_{i j}(i, j) \neq(1,1)(1,2)(2,2)(3,3)$ form a basis of $H^{1}\left(X, \mathscr{E}_{x t^{0}}\left(\Omega_{X}^{1} \mathcal{O}_{X}\right)\right)$. For that purpose, we consider a mapping $\varphi$ from Spec $C[\varepsilon]\left(\varepsilon^{2}=0\right)$ to Spec $\mathcal{O}$ defined by $\varphi^{*} s_{i j}=a_{i j} \varepsilon, a_{11}=a_{12}=a_{22}=a_{33}=0$, $a_{i j} \in C . \quad \varphi^{*} \mathscr{X}$ corresponds to a 1-cocycle $D_{\gamma}=\left\langle a^{t} \gamma, \partial / \partial \zeta\right\rangle$ because $\Gamma$ operates on $\varphi^{*} \operatorname{Proj} R(\tilde{\tau})$ as follows, $\zeta^{\prime}=\zeta+\gamma \varphi^{*} \tau, \zeta=\left(\zeta_{1}, \zeta_{2}, \zeta_{3}\right), \gamma=\left(\gamma_{1}, \gamma_{2}, \gamma_{3}\right) \in \Gamma$ so that $\partial / \partial \varepsilon=a_{13} \gamma_{3}\left(\partial / \partial \zeta_{1}\right)+\left(a_{21} \gamma_{1}+a_{23} \gamma_{3}\right)\left(\partial / \partial \zeta_{2}\right)+\left(a_{31} \gamma_{1}+a_{32} \gamma_{2}\right)\left(\partial / \partial \zeta_{3}\right)$. Each of $a_{13} \gamma_{3}, a_{21} \gamma_{1}+a_{23} \gamma_{3}$ and $a_{31} \gamma_{1}+a_{32} \gamma_{2}$ defines an element of $H^{1}(X, \mathcal{O})$ which is 
contained in the image of $H^{1}(X, C)$ by the canonical homomorphism. Obviously $\gamma_{1}, \gamma_{2}, \gamma_{3}$ form a basis of $H^{1}(X, \mathcal{O})$.

Hence $\left(a_{13} \gamma_{3}, a_{21} \gamma_{1}+a_{23} \gamma_{3}, a_{31} \gamma_{1}+a_{32} \gamma_{2}\right)$ in $H^{1}\left(X, \mathcal{O}_{X}\right)^{\oplus 3}$ gives a basis of $H^{1}\left(X, \mathscr{E} x t^{0}\left(\Omega_{X}^{1} \mathcal{O}_{X}\right)\right)$, which completes the proof. From the existence of a flat family of deformations of $X$ depending on 9 effective parameters we infer $\operatorname{dim} \operatorname{Ext}^{1}\left(\Omega_{X}^{1} \mathcal{O}_{X}\right)=9$. Moreover according to the above proof, $\mathscr{X}$ is locally complete because the Kodaira-Spencer mapping is surjective ([27] Theorem 3.5.), while the inequalities in Lemma 5.6 turn out to be equalities.

In case of $\Sigma_{7}$ we put,

$$
\begin{aligned}
\tau(s)= & \left(\begin{array}{ccc}
0 & 0 & \tau_{13}+s_{13} \\
s_{21} & 0 & 0 \\
\tau_{13}+s_{31} & s_{32} & 0
\end{array}\right)+\sum_{i=1}^{3} e_{i} \log s_{i i} / 2 \pi \sqrt{-1} \\
& +e_{12} \log s_{12} / 2 \pi \sqrt{-1}+e_{23} \log s_{23} / 2 \pi \sqrt{-1}
\end{aligned}
$$

In case of $\Sigma_{8}$ we put,

$$
\tau(s)=\left(\begin{array}{ccc}
0 & 0 & 0 \\
s_{21} & 0 & 0 \\
s_{31} & s_{32} & 0
\end{array}\right)+\sum_{i=1}^{3} e_{i} \log s_{i i} / 2 \pi \sqrt{-1}+\sum_{i<j} e_{i j} \log s_{i j} / 2 \pi \sqrt{-1}
$$

where

$$
\begin{array}{ccc}
e_{1}=\left(\begin{array}{lll}
1 & 0 & 0 \\
0 & 0 & 0 \\
0 & 0 & 0
\end{array}\right), & e_{2}=\left(\begin{array}{lll}
0 & 0 & 0 \\
0 & 1 & 0 \\
0 & 0 & 0
\end{array}\right), & e_{3}=\left(\begin{array}{lll}
0 & 0 & 0 \\
0 & 0 & 0 \\
0 & 0 & 1
\end{array}\right) \\
e_{12}=\left(\begin{array}{rrr}
1 & -1 & 0 \\
-1 & 1 & 0 \\
0 & 0 & 0
\end{array}\right), & e_{23}=\left(\begin{array}{rrr}
0 & 0 & 0 \\
0 & 1 & -1 \\
0 & -1 & 1
\end{array}\right), & e_{13}=\left(\begin{array}{rrr}
1 & 0 & -1 \\
0 & 0 & 0 \\
-1 & 0 & 1
\end{array}\right) .
\end{array}
$$

Let $\tilde{R}(\tau)=\mathcal{O}\left[\tilde{\xi}_{m} \cdot \theta, m \in Z^{3}\right], \mathcal{O}=\mathcal{O}_{0 E}$ with the notations as in the case of $\Sigma_{6}$. Then $\mathscr{X}=\operatorname{Proj} \tilde{R}(\tau) / \Gamma$ is a complete flat family of deformations of $\mathscr{X}_{0}$ depending on 9 effective parameters. In any case we can construct the desired family of deformations of $X$, so we obtain

THEOREM 5.1. $\operatorname{dim} \operatorname{Ext}^{1}\left(\Omega_{X}^{1} \mathcal{O}_{X}\right)=9$ for a SQAV $X$ of $\operatorname{dim} 3(\lambda=1)$.

Remark. We shall point out an interesting fact that $\Sigma$ is one of $\Sigma_{\nu}(\nu=6,7,8)$ then $\operatorname{dim} H^{1}\left(X, \mathscr{E} x t^{0}\left(\Omega_{X}^{1} \mathcal{O}_{X}\right)\right)$ is given by a formula

$$
\operatorname{dim} H^{1}\left(X, \mathscr{E} x t^{0}\left(\Omega^{1}{ }_{X} \mathcal{O}_{X}\right)\right)=g-3 N_{3}+2 N_{2}-N_{1}+N_{0} \quad(g=3)
$$

where $N_{\nu}$ denotes the number of $\nu$-dimensional Delone cells $\bmod \Gamma$. In 
case of $\Sigma_{6}$ we have $N_{0}=1, N_{1}=6, N_{2}=8, N_{3}=3$. And also we have $N_{0}=1, N_{1}=6, N_{2}=9, N_{3}=4$ if $\Sigma=\Sigma_{7}, N_{0}=1, N_{1}=7, N_{2}=12, N_{3}=6$ if $\Sigma=\Sigma_{8}$. Remark that $\Sigma_{\nu}(\nu=6,7,8)$ does not come from lower dimensional $\mathrm{D}-\mathrm{V}$ cones. However this is not the case if $g=4$.

\section{§ 6. Existence of moduli of SQAV $(g \leqq 3)$}

(6.1) In this section we shall show the existence of the coarse moduli of $\operatorname{SQAV}(\lambda=1,2)$ the fine moduli $(\lambda \geqq 3)$ applying a theorem of Artin ([1][5]) and our results obtained in $\S 4,5$.

At first we prepare the preliminary results and define a functor of SQAV.

THEOREM (Artin [1], [3]). Let $F$ be a contravariant functor from $C$-schemes to sets. Then $\boldsymbol{F}$ is represented by a separated algebraic space locally of finite presentation over $\boldsymbol{C}$ if and only if the following conditions hold.

[0] $F$ is a sheaf for the etale topology

[1] $F$ is locally of finite presentation

[2] $F$ is effectively pro-representable

[3] Let $X$ be a $C$-scheme of finite type and let $\xi, \eta \in F(X)$. Then the condition $\xi=\eta$ is represented by a closed subsheme.

[4] Let $X$ be a $C$-scheme of finite type and let $\xi: X \rightarrow F$ be a map. If $\xi$ is formally etale at a point $x \in X$, then it is formally etale in a neighborhood of $x$.

According to Deligne-Rapoport ([5] (p.201,202, p.210 2.6.), $F$ is represented by an algebraic space locally of finite presentation if the conditions [0][1][2][4] and the following (a)(b) are satisfied,

(a) For any $\xi \in F(C)$, let $\xi^{\prime}=\xi \times{ }_{c}$ Spec $C[\varepsilon]\left(\varepsilon^{2}=0\right)$ then $\operatorname{Aut}_{c} \xi=$ Aut $_{C[\varepsilon]} \xi^{\prime}$

(b) For any $\xi \in F(C)$, Aut $c \xi=\{1\}$.

And also the condition [4] holds if $F$ is (locally) effectively pro-represented by complete normal rings of the same Krull dimension.

Notice that in this case $F$ is not necessarily represented by a separated algebraic space ([5] p. 204 Remarque 2.6.)

Definition 6.1. $(g \leqq 4)\left(X, \pi, S, G, H, H^{\prime}, \varepsilon_{j} j=0, \cdots, 2 g, L\right)$ is a principally polarized stable quasi abelian scheme of level $\lambda$ over a $C$ scheme $S$ if 
(0) $\pi$ is a flat proper reduced and surjective morphism

(1) $G$ is an open $S$-subscheme of a $S$-scheme $X$ and a commutative $S$ group scheme operating on $X$ with identity $\varepsilon_{0}$.

(2) $H$ is a $S$-group subscheme of $G$ consisting of all $\lambda$-division points of $G$ and isomorphic with $(Z / \lambda)^{2 g} \times S$. $\quad \varepsilon_{j}$ is a $Z$-basis of $H(j=1, \cdots, 2 g)$.

(3) $H^{\prime}$ is a $S$-group subscheme of $H$ acting freely on $X$, is isomorphic with $(Z / \lambda)^{g} \times S . \quad H^{\prime}$ is generated by $\varepsilon_{g_{+j}}(j=1, \cdots, g)$

(4) $L$ is a relatively ample invertible sheaf on $X$ and $H^{\prime}$ operates on $L$ so that the following diagram commutes.

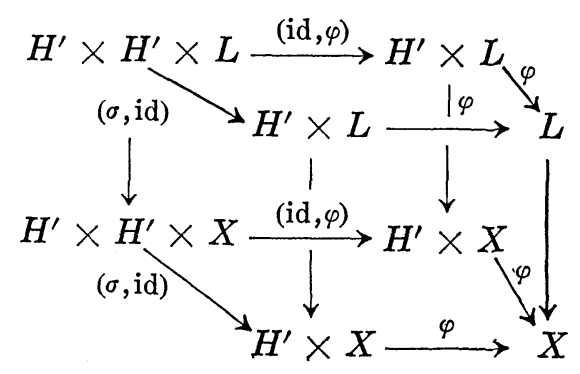

where $\sigma$ is a group mutiplication of $H^{\prime}$ and $\varphi$ is an action of $H^{\prime}$ on $X$. (5) for any geometric point Spec $K$ of $S$ the fiber product over $S$

$$
\left(X \times K, \pi \times K, \operatorname{Spec} K, G \times K, H \times K, H^{\prime} \times K, \varepsilon_{j} \times K, L \times K\right)
$$

is a principally polarized stable quasi-abelian scheme of level $\lambda$ over $K$.

Remark. If $\lambda=1, H=H^{\prime}=\varepsilon$ where $\varepsilon$ denotes the identity of $G$. So we write a SQAS $\xi$ of level 1 over $S$ by $\xi=(X, \pi, S, G, \varepsilon, L)$.

DeFINITION 6.2. Two SQAV's over $S\left(X_{i}, \pi_{i}, S, G_{i}, H_{i}, H_{i}^{\prime}, \varepsilon_{j}^{(i)}, L_{i}\right)(i=$ $1,2)$ are isomorphic to each other if the following condition is satisfied, there exists an $S$-isomorphism $f$ between $X_{1}$ and $X_{2}$

$$
f: X_{1} \rightarrow X_{2}
$$

such that the restriction of $f$ to $G_{1}$ induces an isomorphism between $G_{1}$ and $G_{2}$ and

$$
f^{*} \varepsilon_{j}^{(2)}=\varepsilon_{j}^{(1)}, \quad f^{*} H_{2}=H_{1}, \quad f^{*} H_{2}^{\prime}=H_{1}^{\prime}, \quad\left(f^{*} L_{2}\right) L_{1}^{-1} \in \mathrm{Pic}^{0}\left(X_{1} / S\right) .
$$

DeFinition 6.3. $\quad F_{g}(\lambda)$ is a contravariant functor from $C$-schemes to sets defined by, 
$F_{g}(\lambda)(S)=$ the set of isomorphism classes of stable quasi abelian schemes over $S$.

Let $\mathscr{S}_{g}(\lambda)$ be the sheafification of $F_{g}(\lambda)$ in the etale topology.

Remark. We use here a terminology "a stable quasi abelian scheme" (abbr. SQAS) over $S$ only for an element of $F_{g}(\lambda)$.

Our aim is to prove that $\mathscr{S}_{g}(\lambda)$ satisfies the conditions [0][1][a][b] and is locally effectively pro-represented by complete regular local rings of Krull dimension $\frac{1}{2} g(g+1)$ if $\lambda \geqq 3, g=2,3$.

LEMMA 6.1. $\mathscr{S}_{g}(\lambda)$ is locally of finite presentation.

Proof. Let $\left\{B_{\nu}\right\}$ be an inductive system of $C$-algebras of finite presentation and $B=\lim _{\rightarrow} B_{\nu}$. Take an element $\xi$ of $\mathscr{S}_{g}(\lambda)(B)$. Then $\xi$ is represented by, for an etale covering $S_{\alpha}, \alpha \in I$ of Spec $B$ and SQAS's over $S_{\alpha}\left(X_{\alpha}, \pi_{\alpha}, S_{\alpha}, G_{\alpha}, H_{\alpha}, H_{\alpha}^{\prime}, \varepsilon_{j}^{\alpha}, L_{\alpha}\right)$.

Since $\operatorname{Spec} B$ is quasi-compact (even if $B$ is not neotherian) so we may assume $I$ to be a finite set and moreover $S_{\alpha}$ to be affine. $X_{\alpha}$ is projective over $S_{\alpha}$ hence $G_{\alpha}$ is quasi-projective, and so they are of finite presentation over $S$. And then the group law of $G_{\alpha}$ is of finite presentation (EGA IV 8.13.2).

On the other hand the open immersion and the closed immersion are also locally of finite preseptation (EGA IV 8.10.5) and so on. Hence there exists a $\nu_{0}$ such that $\xi$ comes from an element of $\mathscr{S}_{g}(\lambda)\left(B_{\nu_{0}}\right)$.

Next take two inductive systems $\left\{\xi_{\nu}\right\},\left\{\eta_{\nu}\right\}$ such that $\xi=\eta$ in $\mathscr{S}_{g}(B)$ where $\xi=\lim \xi_{\nu}, \eta=\lim \eta_{\nu}$. Hence there exists a finite etale affine covering $S_{\alpha}$ of $\operatorname{Spec} B$ so that $\xi$ and $\eta$ are represented by $\xi^{\alpha}=\left(X_{\alpha}, \pi_{\alpha}, S_{\alpha}, G_{\alpha}\right.$, $\left.H_{\alpha}, H_{\alpha}^{\prime}, \varepsilon_{j}^{\alpha}, L_{\alpha}\right), \eta^{\alpha}=\left(\tilde{X}_{\alpha}, \tilde{\pi}_{\alpha}, \tilde{S}_{\alpha}, \tilde{G}_{\alpha}, \tilde{H}_{\alpha}, \tilde{H}_{\alpha}^{\prime}, \tilde{\varepsilon}_{j}^{\alpha}, \tilde{L}_{\alpha}\right)$ respectively and $\xi^{\alpha} \cong \eta^{\alpha}$. By assumption all except $L_{\alpha}$ and $\tilde{L}_{\alpha}$ are isomorphic and these isomorphisms are also of finite presentation. (EGA IV 8.8.2.) Since $X_{\alpha}$ is projective over $S_{\alpha}$ and $\pi_{\alpha}$ is cohomologically flat (at least if $g \leqq 4$ ), so $\operatorname{Pic}^{0}\left(X_{\alpha} / S_{\alpha}\right)$ is locally of finite presentation ([1]) there exists a $\nu$ such that $\xi^{\alpha}$ and $\eta^{\alpha}$ are isomorphic in $\mathscr{S}_{g}(\lambda)\left(B_{\nu}\right)$. Thus we have proved ourproposition.

LEMMA 6.2. $\mathscr{S}_{g}(\lambda)(A)=F_{g}(\lambda)(A)$ for a complete local ring $A$.

Proof. An element $\xi$ of $\mathscr{S}_{g}(\lambda)(A)$ is represented by $\xi_{\nu}=\left(X_{\nu}, \pi_{\nu}, S_{\nu}, G\right.$, $\left.H_{\nu}, H_{\nu}^{\prime}, \varepsilon_{j}^{\nu}, L_{\nu}\right)$ with a finite affine etale covering $S_{\nu}$ of Spec $A$. Take an $S_{\nu}$. Since $A$ consists of a unique point as a topological space, $S_{\nu}$ consists of a finite number of points as a topological space. Let $x_{1}^{(\nu)} \cdots x_{k_{\nu}}^{(\nu)}$ be 
all the closed points of $S_{\nu^{\prime}}$ and $A_{j}^{(\nu)}=O_{x_{j} S_{\nu}}$ be the local rings of $S_{\nu}$ at $x_{j}$. Then $A_{j}^{(\nu)}$ is etale over $A$, hence $A_{j}^{(\nu)}$ is isomorphic with $A$, for $A$ is a complete local ring. Hence we can consider another etale covering $T_{\nu, j}$ (=Spec $A_{j}^{(\nu)}$ ) of $\operatorname{Spec} A$, and a pull back $\xi_{\nu, j}$ of $\xi_{\nu}$. Since they coincide with each other over $T_{\nu . j} \times_{\text {Spec } A} T_{\mu i}$, they determine only a SQAV $\xi^{*}$ of level $\lambda$ over $A$, namely, $\xi$ is represented by a SQAV $\xi^{*}$ of level $\lambda$ over $\operatorname{Spec} A$.

The isomorphism between $\xi^{\prime} \mathrm{s}\left(\xi \in \mathscr{S}_{g}(\lambda)(A)\right)$ is nothing but that between $\xi^{*}$ in $F_{g}(\lambda)(A)$. Hence we have the isomorphism between $\mathscr{S}_{g}(\lambda)(A)$ and $F_{g}(\lambda)(A)$ for a complete local ring $A$.

Q.E.D.

(6.2) Let $\xi=\left(X, \pi, S, G, H, H^{\prime}, \varepsilon_{j}, L\right)$ be a SQAS of level $\lambda$ over $S$ $\left(\xi \in F_{g}(\lambda)(S)\right)$. Then we can construct a quotient $\xi \bmod H^{\prime}$ as follows. Since $H^{\prime}$ acts on $X$ and $L$ in a compatible way, so there exists a scheme $Y$ and a projection $f$ from $X$ to $Y$ and an invertible sheaf $L_{Y}$ on $Y$ such that $L=f^{*} L_{Y}$, the following diagram commutes

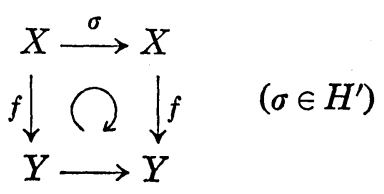

Set $G_{Y}=G / H^{\prime}, \varepsilon_{Y}=f \circ \varepsilon, \pi_{Y}=\pi \circ f^{-1}$ where $\varepsilon$ is the identity section of $G$. Then obviously $\left(Y, \pi_{Y}, S, G_{Y}, \varepsilon_{Y}, L_{Y}\right)$ is a SQAS of level 1 over $S$.

LEMMA 6.3. ( $g \leqq 4)$ Let $\xi=\left(X, \pi, S, G, H, H^{\prime}, \varepsilon_{j}, L\right)$ be a $S Q A S$ of level $\lambda$ and $\eta=\left(X, \pi_{Y}, S, G_{Y}, \varepsilon_{Y}, L_{Y}\right)$ be a $S Q A S$ constructed above. For given $X, \varepsilon_{j}, H^{\prime}$ and $\pi$ there exists only one structure on $X$ as a $S Q A V$ of level $\lambda$.

Proof. Let $X^{\text {reg }}$ be the maximal open subscheme of $X$ such that for any geometric point $K$ of $S, X^{\mathrm{reg}} \times{ }_{S} K$ is smooth over $K$. Let $\mathscr{F}$ be the set of open subschemes $Z$ of $X^{\text {reg }}$ containing $H^{\prime}$ such that for any geometric point $K$ of $S$

$$
\#\left(\text { connected components of } Z_{R}\right)=\lambda^{g^{\prime \prime}}
$$

where $g^{\prime \prime}$ is the rank of an integral matrix associated with $X_{K}$. Then $G$ is the maximal element of $\mathscr{F}$ w.r.t. inclusion. In fact, if $g \leqq 4$, a SQAS over an algebraically closed field $K$ is described explicitly in $\S 2$. From this our claim follows readily. 
The group structure of the connected component $G_{K}^{0}$ of $G_{K}$ containing $\varepsilon_{0}$ is uniquely determined by $\varepsilon_{0}$ and $G_{Y} \times_{S} K$ where $\varepsilon_{0}$ is the identity of $G$. Iu fact since $G_{K}^{0}$ is an extension of a split torus $\left(K^{*}\right)^{g^{\prime \prime}}$ by an abelian variety $A_{K}$. The quotient $A_{K}^{\prime}=A_{K} / H^{\prime} \times G_{K} G_{K}^{0}$ is also an abelian variety. Then $G_{Y} \times{ }_{S} K$ is an extension of a split torus $\left(K^{*}\right)^{g^{\prime \prime}}$ by $A_{K}^{\prime}$. So $A_{K}^{\prime}$ is uniquely determined by $G_{Y} \times_{S} K$, then the group structure $A_{K}$ is uniquely determined by $A_{K}^{\prime}, \varepsilon_{0}$ and $\varepsilon_{j}$, hence by $G_{Y} \times_{S} K$ and $\varepsilon_{0}$. Moreover the group structure of $\left(K^{*}\right)^{g^{\prime \prime}}$ is uniquely determined also by $\varepsilon_{0}$, so that of $G_{K}^{0}$ is uniquely determined by $G_{X} \times{ }_{S} K$ and $\varepsilon_{0}$. The structure of $G_{K}$ is determined by $\varepsilon_{j}$ and $G_{K}^{0}$, so by $G_{X} \times_{S} K, \varepsilon_{j}$ and $\varepsilon_{0}$. (see footnote.)

Let two group structures on $G$ be $G_{1}$ and $G_{2}, f$ be an identity morphism of $G$. Then we have only to prove $f$ is a $S$-group homomorphism from $G_{1}$ to $G_{2}$. Given two points $a, b \in G_{1}(S)$, we have $f(a) f(b)$ $=f(a b)$ over $K$ for any geometric point $K$ of $S$. Since $f(a) f(b)$ and $f(a b)$ are sections over $S$, they coincide over $k(x)$ for any point $x$ of $S$ by the f.p.q.c. descend. So in particular, they coincide simply as subsets in $X$. On the other hand the images of these two sections by the projection from $G$ to $G_{Y}$ are also sections of $G_{Y}$ over $S$, and coincide as sections by assumption. In the etale topology the section of $G$ over $S$ and its image in $G_{Y}$ is isomorphic, therefore the sections $f(a) f(b)$ and $f(a b)$ coincide in the same topology.

$L$ is the pull back of $L_{Y}$, so uniquely determined by $\eta$. Thus we have proved our proposition.

(6.3) Assume $X$ to be a proper reduced $C$-scheme and $L$ an invertible sheaf on $X$. We define a covariant functor $D_{L}$ from the category of complete local rings $\widetilde{C}$ over $C$ to sets by, $D_{L}(A)=$ the set of equivalence classes of a pair $(\mathscr{X}, \mathscr{L})$ such that

(1) $\mathscr{X}$ is a proper flat $A$-scheme with $\mathscr{X}_{0}=X$

(2) $\mathscr{L}$ is an invertible sheaf on $\mathscr{X}$ with $\mathscr{L}_{0}=L$.

where $\left(\mathscr{X}_{1}, \mathscr{L}_{1}\right)$ and $\left(\mathscr{X}_{2}, \mathscr{L}_{2}\right)$ are defined to be equivalent if there exists an $A$-isomorphism $f$ from $\mathscr{X}_{1}$ to $\mathscr{X}_{2}$ such that $\left(f^{*} \mathscr{L}_{2}\right) \mathscr{L}_{1}^{-1} \in \operatorname{Pic}^{0}\left(\mathscr{X}_{1} / A\right)(A)$.

LEMMA 6.4. Let the notations and the assumptions be as above. Suppose that the natural homomorphism from $H^{1}(X, C)$ to $H_{\mathrm{I}}(X, \mathcal{O})$ is surjective. Then $D_{L}$ has a hull. Moreover if the deformation functor $D_{X}$ of $X$ is pro-representable then so is $D_{L}$.

We can prove $G_{Y} \times_{S} K / \operatorname{Im}\left(H \times{ }_{S} G_{K}^{0}\right) \cong G_{K}^{0}$. 
Proof. At first we prove the following

Claim; Let $A, B$ be complete local rings and $\varphi$ be an epimorphism from $B$ to $A$. Suppose $\mathscr{X}$ to be a proper flat B-scheme with $\mathscr{X}_{0}=X$. Denote by $\mathscr{X}_{A}$ the fiber product of $\mathscr{X}$ and $A$ through $\varphi$. Then a canonical homomorphism $\varphi^{*}$ from $H^{1}\left(\mathscr{X}, \mathcal{O}_{\mathscr{Q}}\right)$ to $H^{1}\left(\mathscr{X}_{A}, \mathcal{O}_{\mathscr{x}_{A}}\right)$ is surjective. In fact, since $\mathscr{X}$ is proper over $B, H^{1}\left(\mathscr{X}, \mathcal{O}_{\mathscr{x}}\right)$ is a finite $B$-module. Put $M=$ Coker $\left(H^{1}(\mathscr{X}, B) \rightarrow H^{1}\left(\mathscr{X}, \mathcal{O}_{\mathscr{X}}\right)\right)$. Then we have $M \otimes B / m_{B}=0$ by assumption, where $m_{B}$ denotes the maximal ideal of $B$. Hence by an aid of Nakayama's lemma, we conclude $M=0$. On the other hand, it is obvious that $H^{1}(\mathscr{X}, B)=H^{1}(X, B) \cong H^{1}(X, C) \otimes B, H^{1}(\mathscr{X}, A)=H^{1}(X, C) \otimes A$. Therefore it follows from a commutative diagram

$$
\begin{aligned}
H^{1}(\mathscr{X}, B) & \rightarrow H^{1}\left(\mathscr{X}, \mathcal{O}_{\mathscr{X}}\right) \\
\downarrow & \downarrow \\
H^{1}(\mathscr{X}, A) & \rightarrow H^{1}\left(\mathscr{X}_{A}, \mathcal{O}_{\mathscr{X}_{A}}\right)
\end{aligned}
$$

that $\varphi^{*}$ is surjective (QED of the claim).

According to Schlessinger's theorem ([23] theorem 2.11) we have only to check the conditions $\left(H_{1}\right)\left(H_{2}\right)\left(H_{3}\right)$ in order to prove our lemma. We proceed our proof in the same way as in [23]. We use freely the terminology in [23].

$\left(H_{1}\right)$ Let $u^{\prime}:\left(A^{\prime}, \eta^{\prime}\right) \rightarrow(A, \eta)$ and $u^{\prime \prime}:\left(A^{\prime \prime}, \eta^{\prime \prime}\right) \rightarrow(A, \eta)$ be morphisms of couples where $u^{\prime \prime}$ is surjective. Let $\mathscr{X}^{\prime}, \mathscr{X}, \mathscr{X}^{\prime \prime}$ and $\mathscr{L}^{\prime}, \mathscr{L}, \mathscr{L}^{\prime \prime}$ corresponding schemes and invertible sheaves on them respectively. Namely $\eta^{\prime}=$ $\left(\mathscr{X}^{\prime}, \mathscr{L}^{\prime}\right), \eta=(\mathscr{X}, \mathscr{L})$ and $\eta^{\prime \prime}=\left(\mathscr{X}^{\prime \prime}, \mathscr{L}^{\prime \prime}\right)$.

Then we have isomorphisms $\mathscr{X}^{\prime} \otimes A \cong \mathscr{X}, \mathscr{X}^{\prime \prime} \otimes A \cong \mathscr{X}$ and $\left(\mathscr{L}^{\prime} \otimes A\right) \mathscr{L}^{-1} \in \operatorname{Pic}^{0}(\mathscr{X}),\left(\mathscr{L}^{\prime \prime} \otimes A\right) \mathscr{L}^{-1} \in \operatorname{Pic}^{0}(\mathscr{X})$.

By the above claim there exist invertible sheaves $\hat{\mathscr{L}}^{\prime}, \hat{\mathscr{L}}^{\prime \prime}$ respectively on $\mathscr{X}^{\prime}, \mathscr{X}^{\prime \prime}$ such that $\hat{\mathscr{L}}^{\prime} \otimes A \cong \mathscr{L}, \hat{\mathscr{L}}^{\prime \prime} \otimes A \cong \mathscr{L}$. Put $Z$ with $\mathcal{O}_{Z}=\mathcal{O}_{\mathscr{x}^{\prime}} \times_{\mathscr{O}_{\mathscr{X}}} \mathcal{O}_{\mathscr{X}^{\prime \prime}}$, $|Z|=|X|$ and $\mathscr{N}=\hat{\mathscr{L}}^{\prime} \times_{\mathscr{L}} \hat{\mathscr{L}}^{\prime \prime}, \eta^{\prime \prime \prime}=(Z, \mathscr{N})$. Then we have isomorphisms $\eta^{\prime \prime \prime} \otimes A^{\prime} \cong \eta^{\prime}, \eta^{\prime \prime \prime} \otimes A^{\prime \prime} \cong \eta^{\prime \prime}$, which completes the proof of $\left(H_{1}\right)$.

$\left(H_{2}\right) \quad$ Next let $\left(\mathscr{X}_{1}, \mathscr{L}_{1}\right)$ and $\left(\mathscr{X}_{2}, \mathscr{L}_{2}\right)$ be elements of $D_{L}\left(A^{\prime} \times{ }_{c} A^{\prime \prime}\right), A^{\prime \prime}=C[\varepsilon]$ $\left(\varepsilon^{2}=0\right)$. If $\mathscr{X}_{1} \otimes A^{\prime} \cong \mathscr{X}_{2} \otimes A^{\prime}, \mathscr{X}_{1} \otimes A^{\prime \prime} \cong \mathscr{X}_{2} \otimes A^{\prime \prime}$ and through these isomorphisms $\mathscr{L}_{1} \otimes A^{\prime}=\mathscr{L}_{2} \otimes A^{\prime} \bmod \operatorname{Pic}^{0}\left(\mathscr{X}_{1} \otimes A^{\prime}\right)$ and $\mathscr{L}_{1} \otimes A^{\prime \prime}=\mathscr{L}_{2} \otimes A^{\prime \prime}$ $\bmod \operatorname{Pic}^{0}\left(\mathscr{X}_{1} \otimes A^{\prime \prime}\right)$, then there exists by $\left(H_{1}\right)$ an invertible sheaf $\mathscr{L}$ on $\mathscr{X}_{1}$ such that

$$
\mathscr{L} \otimes A^{\prime}=\left(\mathscr{L}_{1} \otimes A^{\prime}\right)\left(\mathscr{L}_{2} \otimes A^{\prime}\right)^{-1}, \quad \mathscr{L} \otimes A^{\prime \prime}=\left(\mathscr{L}_{1} \otimes A^{\prime \prime}\right)\left(\mathscr{L}_{2} \otimes A^{\prime \prime}\right)^{-1}
$$

Since $\mathscr{L}_{0}=\mathcal{O}_{X}, \mathscr{L}$ is an element of $\operatorname{Pic}^{0}(Z)$ where $Z$ is defined by $\mathcal{O}_{Z}=$ 
$\mathcal{O}_{x_{1}} \times_{O_{X}} \mathcal{O}_{x_{2}},|Z|=|X|$. In fact, since $\operatorname{Pic}^{0}\left(Z / A^{\prime \prime \prime}\right)=\operatorname{Pic}^{0}\left(Z_{a n} / A^{\prime \prime \prime}\right)$ where $Z_{a n}$ denotes an analytic space associated with $Z, A^{\prime \prime \prime}=A^{\prime} \times{ }_{c} A^{\prime \prime}$, we have only to prove the assertion in the analytic case. From a commutative diagram

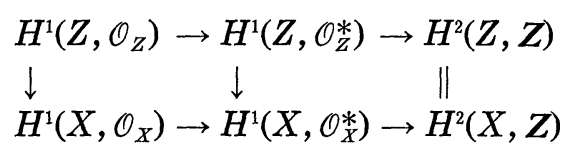

we infer that any invertible sheaf $\mathscr{L}$ on $Z$ whose restriction on $X$ is an element of $\operatorname{Pic}^{0}(X)$ is projected to zero in $H^{2}(Z, Z)$, i.e., comes from $H^{1}\left(Z, \mathcal{O}_{Z}\right)$.

Therefore changing $\mathscr{L}_{1}$ into $\mathscr{L}_{1} \otimes \mathscr{L}^{-1}$, we have isomorphisms

$$
\begin{aligned}
& \mathscr{X}_{1} \otimes A^{\prime} \cong \mathscr{X}_{2} \otimes A^{\prime}, \quad \mathscr{X}_{1} \otimes A^{\prime \prime} \cong \mathscr{X}_{2} \otimes A^{\prime \prime} \\
& f \quad g \\
& \mathscr{L}_{1} \otimes A^{\prime} \cong f^{*} \mathscr{L}_{2} \otimes A^{\prime}, \quad \mathscr{L}_{1} \otimes A^{\prime \prime} \cong g^{*} \mathscr{L}_{2} \otimes A^{\prime \prime} .
\end{aligned}
$$

By the same argument as in [23], we conclude that $\left(\mathscr{X}_{1}, \mathscr{L}_{1}\right)$ and $\left(\mathscr{X}_{2}, \mathscr{L}_{2}\right)$ are isomorphic if $A^{\prime \prime}=C[\varepsilon], A=C$, which proves $\left(H_{2}\right)$.

$\left(H_{3}\right)$ is obvious since $D_{L}$ is a subfunctor of the deformation functor. The last assertion follows readily from Th. 2.11 and Lemma 3.8 in [23].

Q.E.D.

LEMma 6.5. Let $X$ be a proper reduced k-scheme and $L$ be an invertible sheaf on $X$. Suppose that $\mathscr{X}$ is flat and proper over $k[\varepsilon], \varepsilon^{2}=0$ and $\mathscr{X}_{0}=X$. Moreover we assume $\mathscr{X}$ be an element of $H^{1}\left(X, \mathscr{E} x t^{0}\left(\Omega_{X}^{1} \mathcal{O}_{X}\right)\right)$ through the canonical isomorphism between $\operatorname{Ext}^{1}\left(\Omega_{X}^{1} \mathcal{O}_{X}\right)$ and the tangent space of a deformation functor of $X$. Then an obstruction o(X, $L)$ to extend $L$ to the whole $\mathscr{X}$ is given by the cup-product of $\mathscr{X}$ and $c(L)$, and $o(\mathscr{X}, L)$ is an element of $H^{2}\left(X, \mathcal{O}_{X}\right)$ where $c$ is a canonical homomorphism induced from the homomorphism from $\mathcal{O}^{*}$ into $\Omega^{1}$ defined by $c(a)=d a / a$, so $c(L)$ is an element of $H^{1}\left(X, \Omega_{X}^{1}\right)$.

Proof. This lemma is well-known, however for the later use we recall the outline of the proof. Take an open affine covering $U_{j}$ of $X$ and an open covering $\mathscr{U}_{j}$ of $\mathscr{X}$ such that $\mathscr{U}_{j} \times_{\mathscr{x}} X=U_{j}$. Let $L$ be defined by a 1-cocycle $\left\{f_{j k}\right\}, f_{j k} \in \Gamma\left(U_{j k}, \mathcal{O}^{*}\right)$. And assume $\mathscr{X}$ to correspond to a 1-cocycle $D_{j k}, D_{j k} \in \Gamma\left(U_{j k} \mathscr{E}_{x t}{ }^{0}\left(\Omega_{X}^{1} \mathcal{O}_{X}\right)\right)$.

Now assume that there exists an extension $\mathscr{L}$ of $L$, say, defined by a 1-cocycle $F_{j k}, F_{j k} \in \Gamma\left(\mathscr{U}_{j k}, \mathcal{O}^{*}\right)$ such that $F_{j k}(0)=f_{j k}$. We write $F_{j k}^{1}=$ 
$\partial F_{j k} / \partial \varepsilon, F_{j k}^{0}=f_{j k}$. Then one-cocycle $F_{j k}$ satisfies the equality $F_{i j} F_{j k}=$ $F_{i k}$. Differentiating this equality with $\varepsilon$, we have,

$$
F_{i j}^{0} F_{j k}^{1}+F_{i j}^{1} F_{j k}^{0}+\left(D_{j k} F_{i j}^{0}\right) F_{j k}^{0}=F_{i k}^{1}
$$

Hence we conclude a 2-cocycle $\left(1 / F_{i j}^{0}\right) D_{j k} F_{i j}^{0}$ is cohomologous to zero in $H^{2}\left(X, \mathcal{O}_{X}\right)$.

The converse is left to the reader.

Remark. The above obstruction will be often denoted by $o(\partial / \partial \varepsilon, L)$.

(6.4) Next we shall calculate $o(\partial / \partial \varepsilon, L)$ for SQAV of level 1 using the above result. Because of the similarity of calculations we deal with only SQAV's of dimension 2.

case 1 )

Assume $X$ to be a principally polarized abelian surface. Let the period matrix of $X$ be $(1, \tau), \tau=\left(\begin{array}{ll}\tau_{11} & \tau_{12} \\ \tau_{12} & \tau_{22}\end{array}\right)$. Then a complete family of deformations is given by setting $\tau(s)=\left(\begin{array}{ll}\tau_{11}+s_{11} & \tau_{12}+s_{12} \\ \tau_{12}+s_{21} & \tau_{22}+s_{22}\end{array}\right),\left|s_{i j}\right|<\varepsilon$. Consider a mapping $\varphi$ from Spec $C[\varepsilon], \varepsilon^{2}=0$ into $s_{i j}$-space defined by $\varphi^{*} s_{i j}=a_{i j} \varepsilon$ so that we may obtain a flat family of deformations with period $\tau(\varepsilon)=\tau+\left(\begin{array}{ll}a_{11} & a_{12} \\ a_{21} & a_{22}\end{array}\right) \varepsilon$ over Spec $C[\varepsilon]$.

Then $o(\partial / \partial \varepsilon, L)$ vanishes if and only if $a_{12}=a_{21}$. We shall give a proof of it.

An invertible sheaf $L$ is given by a 1-cocycle $\theta_{\gamma}=e\left(-\frac{1}{2} \gamma \tau^{t} \gamma-\gamma^{t} \zeta\right)$. Denoting the global coordinate of the generic fiber by $\zeta(\varepsilon)$, then we can write a transformation induced by $\gamma$ as follows,

$$
\zeta^{\prime}(\varepsilon)=\zeta+\tau(\varepsilon)^{t} \gamma=\zeta^{\prime}+\left(a^{t} \gamma\right) \varepsilon
$$

where $a=\left(a_{i j}\right), \zeta^{\prime}=\zeta^{\prime}(0)$. Hence we have $D_{r}=(\partial / \partial \varepsilon)_{\gamma}=\left\langle a^{t} \gamma, \partial / \partial \zeta\right\rangle=$ $\sum_{i, j} a_{i j} \gamma_{j}\left(\partial / \partial \zeta_{i}\right)$. The cup-product of $D_{r}$ and $\theta_{r^{\prime}}$ is given by a 2-cocycle $C_{r r^{\prime}}=-\gamma^{\prime} a^{t} \gamma$, where $\gamma=\left(\gamma_{1}, \gamma_{2}\right), \gamma^{\prime}=\left(\gamma_{1}^{\prime}, \gamma_{2}^{\prime}\right)$.

However $\gamma_{1} \gamma_{1}^{\prime}, \gamma_{2} \gamma_{2}^{\prime},\left(\gamma_{1}+\gamma_{2}\right)\left(\gamma_{1}^{\prime}+\gamma_{2}^{\prime}\right)$ is cohomologous to zero because each of them is the cup-product of a 1-cocycle in $H^{1}(\Gamma, C)$ and itself. On the other hand, $\gamma_{1} \gamma_{2}^{\prime}$ is not cohomologous to zero, so $o(\partial / \partial \varepsilon, L)$ vanishes if and only if $a_{12}=a_{21}$.

case 2)

Assume $X$ be a SQAV of the second type (see (4.2.)). Then the corresponding local period matrix to $X$ is given by $\tau(s)=\left(\begin{array}{cc}\tau_{11} & \tau_{12} \\ \tau_{12} & 0\end{array}\right)+$ 
$\left(\begin{array}{ll}0 & 0 \\ 0 & 1\end{array}\right) \log s / 2 \pi \sqrt{-1}$. And a complete family $\mathscr{X}$ of deformations of $X$ is given by setting $\tilde{\tau}(s)=\left(\begin{array}{cc}\tau_{11}+s_{11} & \tau_{12}+s_{12} \\ \tau_{12}+s_{21} & 0\end{array}\right)+\left(\begin{array}{ll}0 & 0 \\ 0 & 1\end{array}\right) \log s_{22} / 2 \pi \sqrt{-1},\left(\left|s_{i j}\right|<\varepsilon\right)$, $\mathscr{X}=\operatorname{Proj} \tilde{R}(\tilde{\tau}) / \Gamma$ ((2.4.) Remark).

Consider a mapping $\varphi$ of Spec $C[\varepsilon]$ into $s_{i j}$-space defined by $\varphi^{*} s_{i j}=$ $a_{i j},(i j) \neq(22), \varphi^{*} s_{22}=0$, so that we have a flat family $\varphi^{*} \mathscr{X}$ over Spec $C[\varepsilon]$.

Then we may consider $\varphi^{*} \mathscr{X}$ corresponds to a 1-cocycle $D_{r}=\left\langle a^{t} \gamma, \partial / \partial \zeta\right\rangle$ symbolically, where $a=\left(\begin{array}{cc}a_{11} & a_{12} \\ a_{21} & 0\end{array}\right)$. By the same argument as above we have a 2-cocycle $o(\partial / \partial \varepsilon, L)=-\gamma^{\prime} a^{t} \gamma$, therefore $o(\partial / \partial \varepsilon, L)$ vanishes if and only if $a_{12}=a_{21}$.

case 3)

Assume $X$ be a SQAV of the third type whose corresponding local period matrix $\tau(s)$ given by $\tau(s)=\left(\begin{array}{cc}0 & \tau_{12} \\ \tau_{12} & 0\end{array}\right)+\left(\begin{array}{cc}1 & 0 \\ 0 & 1\end{array}\right) \log s / 2 \pi \sqrt{-1}$. And a complete family $\mathscr{X}$ of deformations of $X$ is given by setting $\tilde{\tau}(s)=$ $\left(\begin{array}{cc}0 & \tau_{12}+s_{12} \\ \tau_{12}+s_{21} & 0\end{array}\right)+\left(\begin{array}{ll}1 & 0 \\ 0 & 0\end{array}\right) \log s_{11} / 2 \pi \sqrt{-1}+\left(\begin{array}{ll}0 & 0 \\ 0 & 1\end{array}\right) \log s_{22} / 2 \pi \sqrt{-1}, \mathscr{X}=$ $\operatorname{Proj} \tilde{R}(\tilde{\tau}) / \Gamma$. Consider a pull back $\varphi^{*} \mathscr{X}$ where $\varphi^{*} s_{i j}=a_{i j} \varepsilon(i \neq j), \varphi^{*} s_{i i}=0$. Then $D_{r}=a_{12} \gamma_{2} w_{1}\left(\partial / \partial w_{1}\right)+a_{21} \gamma_{1} w_{2}\left(\partial / \partial w_{2}\right)$ where $w_{i}=e\left(\zeta_{i}\right)$. Also in this case, calculating $o(\partial / \partial \varepsilon, L)$, we can show that $o(\partial / \partial \varepsilon, L)$ vanishes if and only if $a_{12}=a_{21}$.

case 4)

Assume $X$ be a SQAV of the fourth type, whose corresponding local period matrix is given by $\tau(s)=\left(\begin{array}{rr}2 & -1 \\ -1 & 1\end{array}\right) \log s / 2 \pi \sqrt{-1}$. And also by setting $\tilde{\tau}(s)=\left(\begin{array}{cc}0 & 0 \\ s_{21} & 0\end{array}\right)+\left(\begin{array}{ll}1 & 0 \\ 0 & 0\end{array}\right) \log s_{11} / 2 \pi \sqrt{-1}+\left(\begin{array}{rr}1 & -1 \\ -1 & 1\end{array}\right) \log s_{12} / 2 \pi \sqrt{-1}$ $+\left(\begin{array}{ll}0 & 0 \\ 0 & 1\end{array}\right) \log s_{22} / 2 \pi \sqrt{-1}$, we obtain a locally complete family $\mathscr{X}$ of deformations of $X . \quad \mathscr{X}=\operatorname{Proj} \tilde{R}(\tilde{\tau}) / \Gamma$. Consider a pull-back $\varphi^{*} \mathscr{X}$ where $\varphi^{*} s_{21}=$ $a_{21} \varepsilon, \varphi^{*} s_{i j}=0$ (otherwise). Then $D_{r}=a_{21} \gamma_{1} w_{2}\left(\partial / \partial w_{2}\right)$ where $w_{2}=e\left(\zeta_{2}\right)$ and $o(\partial / \partial \varepsilon, L)=-a_{21} \gamma_{1} \gamma_{2}^{\prime}$, hence $o(\partial / \partial \varepsilon, L)$ vanishes if and only if $a_{21}=0$.

In any case, there is a polarized family of deformations of $X$ over a complete regular local ring of Krull dimension 3, and this family is formally complete as for the functor $D_{L}$ defined in (6.3.) in view of Corollarly 2.2 (ii), (4.2.), Lemma 6.4. and [23] Th. 2.11., hence prorepresents $D_{L}$. 
In the case of dimension 3 , by quite similar calculations, we can prove the pro-representability of $D_{L}$.

Summing up these results we have,

THEOREM 6.1. $\quad D_{L}$ is effectively pro-representable for a $\operatorname{SQAV}(X, L)$ of level 1 defined over $C . \quad(g=2,3)$

COROLLARY OF THEOREM 6.1. $\quad \mathscr{S}_{g}(\lambda)$ is locally effectively pro-represented by complete regular local rings of dimension $\frac{1}{2} g(g+1) . \quad(g=2,3)$

Proof. Let $\xi_{0}$ be a SQAS of level $\lambda$ over $C, \xi_{0}=\left(X_{0}, G_{0}, H, H^{\prime}, \varepsilon_{j}, L_{0}\right)$ $\mathscr{S}_{g}(\lambda)(C)\left(=F_{g}(\lambda)(C)\right)$ and $\eta_{0}$ be a SQAS of level 1 obtained from $\xi_{0}$ by taking a quotient, $\eta_{0}=\left(Y_{0}, G_{Y_{0}}, \varepsilon_{0}, L_{Y_{0}}\right)$. ((6.2.))

Then we define covariant functors $\mathscr{S}_{g}(\lambda)\left(\xi_{0}\right), \mathscr{S}_{g}(1)\left(\eta_{0}\right)$ from the category of complete local rings to sets by,

$$
\begin{aligned}
& \mathscr{S}_{g}(\lambda)\left(\xi_{0}\right)(A)=\left\{\xi \in \mathscr{S}_{g}(\lambda)(A) ; \xi \times{ }_{A} A / m_{A} \cong \xi_{0}\right\} \\
& \mathscr{S}_{g}(1)\left(\eta_{0}\right)(A)=\left\{\eta \in \mathscr{S}_{g}(1)(A) ; \eta \times{ }_{A} A / m_{A} \cong \eta_{0}\right\}
\end{aligned}
$$

where $m_{A}$ denotes the maximal ideal of $A$. $D_{L_{\mathrm{Y}_{0}}}$ is effectively prorepresented by a complete regular local ring $A$ of dimension $\frac{1}{2} g(g+1)$ and a flat polarized family $\mathscr{Y}$ of deformations of $Y_{0}$ over $A$ constructed before. Then it's easy to check that $\mathscr{Y}$ is also a SQAS over $A$. Hence any element $\eta$ of $D_{L_{0}}(B)$ is also a SQAS over $B$ for any complete local ring $B$. Therefore $\mathscr{S}_{g}(1)\left(\eta_{0}\right)$ coincides with $D_{L_{Y_{0}}}$ because according to Lemma $6.2 \mathscr{S}_{g}(1)\left(\eta_{0}\right)(B)=F_{g}(1)\left(\eta_{0}\right)(B)$ for a complete local ring $B$ where $F_{g}(1)\left(\eta_{0}\right)$ is defined in the same way as $\mathscr{S}_{g}(1)\left(\eta_{0}\right)$, which proves the effective pro-representability of $\mathscr{S}_{g}(1)\left(\eta_{0}\right)$.

As seen in (6.2.), we can define a SQAS $\eta$ of level 1 for a SQAS $\xi$ of level $\lambda$, which we denote $\eta=\psi(\xi)$. Now we shall prove the mapping $\psi$ from $\mathscr{S}_{g}(\lambda)\left(\xi_{0}\right)(B)$ to $\mathscr{S}_{g}(1)\left(\eta_{0}\right)(B)$ is bijective for any complete local ring $B$. Any element $\eta$ of $\mathscr{S}_{g}(1)\left(\eta_{0}\right)(B)$ is induced from $(\mathscr{Y}, A)$ and there exists an unramified covering $\mathscr{X}$ of $\mathscr{Y}$ which is also a SQAS of level $\lambda$ over $A$ and $\mathscr{X} / H^{\prime}=\mathscr{Y}$, hence $\psi$ is surjective. The injectivity of $\psi$ follows easily from Lemma 6.3.

Q.E.D.

THEOREM 6.2. (i) $\mathscr{S}_{g}(\lambda)$ is represented by a smooth C-algebraic space $S_{g}^{*}(\lambda)$. Moreover the greatest separated quotient $S_{g}(\lambda)$ of $S_{g}^{*}(\lambda)$ exists and is isomorphic with Igusa monoidal transform $\widetilde{\Xi}_{g}^{*}(\lambda)$ of Satake compactification of the Siegel modular space of level $\lambda . \quad(g=2,3, \lambda \geqq 3)$ 
(ii) $\widetilde{\Im}_{g}^{*}(\lambda)(\lambda=1$ or 2$)$ has the following property;

(1) there exists a unique morphism $f$ from $S$ to $\widetilde{\Xi}_{g}^{*}(\lambda)$ in the category of algebraic spaces for a given $S Q A S$ over $S$ functorially.

(2) For any separated $C$-algebraic space $N$ enjoying the property (1), a unique morphism $g$ from $\widetilde{\Im}_{g}^{*}(\lambda)$ to $N$ is defined in such a way that the following diagram commutes, for any $S Q A S \eta$ over $S$

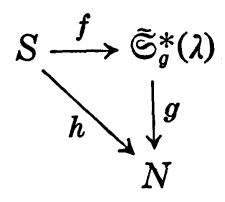

Proof of (i). According to a generalization of Artin's theorem by Deligne-Rapoport (6.1.), it remains to show the conditions (a)(b).

Take a SQAS $\xi$ of level $\lambda$ over $C$, then we can construct $\eta$ of level 1 as before. An automorphism of $\xi^{\prime}=\xi \times{ }_{c} C[\varepsilon],\left(\varepsilon^{2}=0\right)$ induces that of $\eta^{\prime}=\eta \times_{C} C[\varepsilon]$ by definition. By virtue of Theorem 2.1. $\operatorname{Aut}_{C} \eta=\operatorname{Aut}_{C[\varepsilon]} \eta^{\prime}$ because $\eta^{\prime}$ has a section over $C[\varepsilon]$. Hence we have $\operatorname{Aut}_{C} \xi=\operatorname{Aut}_{C[\varepsilon]} \xi^{\prime}$, thus (a) is proved.

Next we shall prove (b). Let $\xi=\left(X, G, H, H^{\prime}, \varepsilon_{j}, L\right) \in \mathscr{S}_{g}(\lambda)(C) . \quad X^{\mathrm{reg}}$ $=X-\operatorname{sing} X$ consists of disjoint union of a split torus bundle over a fixed principally polarized abelian variety $A$ with level $\lambda$ structure. By the condition (B), any automorphism $\sigma$ of $\xi$ induces that of any connected component of $X^{\text {reg }}$, moreover a unique automorphism $\sigma^{\prime}$ of $A$ as a principally polarized abelian variety with level $\lambda$ structure. Hence if $\lambda \geqq 3$, $\sigma^{\prime}$ is an identity. Therefore $\sigma$ operates on $X$ preserving all the fibers. However on the fiber of $X^{\mathrm{reg}}$ at the zero of $A, \sigma$ has $\lambda$-division points as fixed points, so if $\lambda \geqq 3, \sigma$ equals to the identity. (proposition 1.2) So there exists an algebraic space $S_{g}^{*}(\lambda)$ locally of finite presentation which represents $\mathscr{S}_{g}(\lambda)$. The smoothness of $S_{g}^{*}(\lambda)(\lambda \geqq 3)$ follows from Corollary of Theorem 6.1. and (b).

(6.5) We are still on the way of the proof of our theorem.

Now we shall prove the second assertion of (i) that $\widetilde{\Xi}_{g}^{*}(\lambda)$ is the greatest separated quotient of $S_{g}^{*}(\lambda)(\lambda \geqq 3, g=2,3)$.

We have only to consider the problem in the analytic category ([2] Th. 1.12). At first we define an analytic mapping $f$ from $S_{g}^{*}(\lambda)$ to $\widetilde{\Im}_{g}^{*}(\lambda)$ as follows. Let $\xi$ be a SQAS of level $\lambda$ over $C$ and its local period 
matrix be $\tau(s)=\tau_{0}(s)+B \log s / 2 \pi \sqrt{-1}, B=\left(\begin{array}{cc}0 & 0 \\ 0 & B^{\prime \prime}\end{array}\right)\left(B^{\prime \prime}>0\right)$. We may assume that for a Delone $g^{\prime \prime}$ cell $\sigma$ w.r.t. $B^{\prime \prime}, g^{\prime \prime}=\operatorname{rank} B$ the level $\lambda$ structure $\varepsilon_{1}, \cdots, \varepsilon_{2 g}$ on $\xi$ is defined as in (2.4.). Because of the structure of $\widetilde{\Im}_{g}^{*}(\lambda)((3.3)) \lim _{s \rightarrow 0} \tau(s)$ exists, so we can define $f(\xi)=\lim _{s \rightarrow 0} \tau(s)$.

This mapping is obviously an extension of an isomorphism from the fine moduli of principally polarized abelian schemes with level $\lambda$ structures to the Siegel modular space of level $\lambda$.

It is easy to check that $f$ is an analytic mapping ((3.3), (4.2)(5.6.)). Moreover we notice by definition $\#\left(f^{-1}(f(\xi))=\right.$ the number of Delone $g^{\prime \prime}$ cells modulo translations by $\Gamma$. Since $\widetilde{\Im}_{g}^{*}(\lambda)$ is separated our second statement of (i) is completely proved. (see supplement)

Next we turn to the proof of (ii). $(\lambda=1) S p(g Z) / S p(g Z)(k)$ operates on $\widetilde{\Im}_{g}^{*}(k) . \quad(k \geqq 3)$ and its quotient space $\widetilde{\Im}_{g}^{*}(1)$ is defined as an algebraic space indepently of the choice of $k . \widetilde{\Im}_{g}^{*}(1)$ is also separated.

Let $S$ be a $C$-scheme of finite type, $\eta$ be an element of $\mathscr{S}_{g}(1)(S)$ i.e. for an etale covering $S_{\alpha}$ of $S$, SQAS $\eta_{\alpha}=\left(X_{\alpha}, \pi_{\alpha}, S_{\alpha}, G_{\alpha}, \varepsilon_{\alpha}, L_{\alpha}\right)$ over $S_{\alpha}$ are given where $S_{\alpha}$ is also a $C$-scheme of finite type. For any closed point $x_{0}$ of $S_{\alpha}$, the geometric fiber of $\xi_{\alpha}$ at $x_{0}$ is a SQAS over $C$. Let $\hat{X}_{\alpha}$ be the formal completion along the fiber $\left(X_{\alpha}\right)_{x_{0}}$ and $\hat{S}_{\alpha}$ be the completion of $S_{\alpha}$ by the maximal ideal of $\mathcal{O}_{x_{0} S_{\alpha}}$. Then by the (effective) pro-representability (Corollary of Theorem 6.1.) $\hat{X}_{\alpha}$ is isomorphic with Proj $R / \lambda \Gamma$ over $\operatorname{Spec} \mathcal{O}_{x_{0} \hat{S}_{\alpha}}=\operatorname{Spec} \widehat{\mathcal{O}}_{x_{0} S_{\alpha}}$. So there exists a SQAS $\xi$ of level $k(k \geqq 3)$ such that $\eta$ is a quotient of $\xi$ by $H^{\prime}$, so that we may have a morphism from $\hat{S}_{\alpha}$ to the moduli space $S_{g}^{*}(k)$ up to its level structure. Hence this defines a unique morphism from Spec $\hat{\mathscr{O}}_{x_{0} S}$ to $\widetilde{S}_{g}^{*}(k)$ and consequently by an aid of Artin's approximation theorem ([2]), we have a morphism from the henselization $\operatorname{Spec} \tilde{\mathscr{O}}_{x_{0} S}$ of $\operatorname{Spec} \mathcal{O}_{x_{0} S}$ to $\widetilde{\Im}_{g}^{*}(1)$. Thus we obtain a unique morphism from $S$ to $\widetilde{\Im}_{g}^{*}(1)$ as algebraic spaces.

If another separated algebraic space $N$ admits this property, i.e., for any element $\xi$ of $\mathscr{S}_{g}(1)(S)$ there exists a unique morphism from $S$ to $N$ as algebraic spaces, then we have a unique morphism $g$ from $\widetilde{\Im}_{g}^{*}(1)$ to $N$. In fact, let $x_{0}$ be a closed point of $\widetilde{\Im}_{g}^{*}(1)$ and $\mathcal{O}_{x_{0}}$ be its local ring. Then according to the proof of effective pro-representability of $\mathscr{S}_{g}(1)$ there exists a finite Galois covering $\operatorname{Spec} \mathcal{O}^{\prime}$ of $\operatorname{Spec} \mathcal{O}_{x_{0}}$ on which a SQAS $\xi$ of level 1 over $\mathcal{O}^{\prime}$ is defined uniquely up to level structures. Here we may assume $\left(\xi, \mathcal{O}^{\prime}\right)$ is a local universal family of SQAS. A morphism $g$ from $\operatorname{Spec} \mathcal{O}^{\prime}$ to $N$ can be defined by this $\xi$. 
We must prove $g$ is invariant by the action of a Galois covering group $G_{x_{0}}$ of Spec $\mathcal{O}^{\prime}$ over $\operatorname{Spec} \mathcal{O}$. If $x_{0}$ is a point of the Siegel space $\widetilde{S}_{g}^{*}(1) g$ is invariant under $G_{x_{0}}$. Let $x_{0}$ be a point of $\widetilde{\varsigma}_{g}^{*}(1)-\widetilde{S}_{g}^{*}(1)$, and $\sigma$ be a covering transformation of $\operatorname{Spec} \mathcal{O}^{\prime}$ over $\operatorname{Spec} \mathcal{O}$. Then $g$ and $g \circ \sigma$ are morphisms from Spec $\mathcal{O}^{\prime}$ to $N$, and coincide with each other on Spec $K^{\prime}\left(K^{\prime}\right.$ : the quotient field of $\left.\mathcal{O}^{\prime}\right)$ because $\widetilde{S}_{g}^{*}(1)$ is a Zariski open dense subset of $\widetilde{\Im}_{g}^{*}(1)$.

Since $N$ is separated, $g=g \circ \sigma$ on Spec $\mathcal{O}^{\prime}$ which implies $g$ defines a morphism $\bar{g}$ from $\widetilde{\Im}_{g}^{*}(1)$ to $N$. The remaining assertion is trivial.

In the case of $\lambda=2$, we can proceed our proof in the same way as above.

Q.E.D.

Supplement to the proof of theorem 6.2. (i)

Let $N$ be a separated algebraic space of finite type over $C$, and $\varphi$ be a morphism from $S_{g}^{*}(\lambda)$ to $N$. Then we must show that there exists a morphism $h$ from $\mathfrak{S}_{g}^{*}(\lambda)$ to $N$ such that $h \circ f=\varphi$.

Let $P$ be a point of $\mathfrak{S}_{g}^{*}(\lambda), Q$ be one of its inverse image $P$ by $f$. Since $S_{g}^{*}(\lambda)$ and $\mathcal{S}_{g}^{*}(\lambda)$ are locally isomorphic, a morphism $h$ is defined near $Q$. We shall show $h$ is independent of the choice $Q$. If $P$ is contained in the Siegel space $\widetilde{S}_{g}^{*}(\lambda)$, then $Q$ is unique, so there's nothing to prove. Assume that $P$ is not contained in $\bar{\Im}_{g}^{*}(\lambda)$ and $Q_{1}, Q_{2}$ be two points of $f^{-1}(P)$. Let $\mathcal{O}$ be the local ring of $\widetilde{\Im}_{g}^{*}(\lambda)$ at $P$. Then by the above consideration two mapping $h_{1}, h_{2}$ defined at $P$ coincide with each other on Spec $K$ where $K$ is the quotient field of $\mathcal{O}$. Since $N$ is separated, $h_{1}$ and $h_{2}$ coincide all over Spec $\mathcal{O}$. Thus it is proved that $\widetilde{\Phi}_{g}^{*}(\lambda)$ is the greatest separated quotient of $S_{g}^{*}(\lambda)$.

\section{§ 7. Further remarks}

(7.1) We shall give a brief comment on Deligne's example in [13]. Let $X_{2}$ be the fiber $\left(X_{\tau_{0}}\right)_{p}$ at $p:\left(s_{12}^{-1}, s_{11} s_{12}, s_{12} s_{22}\right)=(0,0,0)$ in the case where $\lambda \geqq 3$ (see (3.3.)). $(Z / \lambda)^{2}$ operates on $X_{2}$ so that we have a quotient scheme $X$ of $X_{\lambda}$ by $(Z / \lambda)^{2}$. Obviously $X$ is independent of $\lambda$, which is nothing but the closed fiber of Deligne's example. Notice that $X$ is a union of a projective plane blown up at three vertices and two copies of a projective plane.

As for this $X$, we have $\left.\operatorname{dim} H^{0}\left(X, \mathscr{E}_{x t^{0}} \Omega_{X}^{1} \mathcal{O}_{X}\right)\right)=2, \operatorname{dim} H^{1}\left(X, \mathscr{E} x t^{0}\left(\Omega_{X}^{1} \mathcal{O}_{X}\right)\right)$ $=2, \operatorname{dim} H^{2}\left(X, \mathscr{E}_{x t^{0}}\left(\Omega_{X}^{1} \mathcal{O}_{X}\right)\right)=0$ and $H^{0}\left(X, \mathscr{E} x t^{1}\left(\Omega_{X}^{1} \mathcal{O}_{X}\right)\right)=3$, consequently $\operatorname{dim} \operatorname{Ext}^{1}\left(\Omega_{X}^{1} \mathcal{O}_{X}\right)=5$. Since a canonical homomorphism $H^{1}(X, C) \rightarrow H^{1}\left(X, \mathcal{O}_{X}\right)$ 
is surjective and $\operatorname{dim} H^{2}\left(X, \mathcal{O}_{X}\right)=1$, a flat polarized family of deformations of $X$ with 4 parameters exists. In fact $X$ has apparently two parameters for gluing, one of which prevents polarization from extending.

Moreover we remark that in the Deligne's example one of two projective planes of $X$ can be blown down to one normal point so that $X$ becomes our stable quasi abelian variety, i.e., two copies of a projective plane, and the total space becomes a local universal polarized family of deformations given in (4.2.). Therefore it is conjectured that a stable quasi abelian variety has in a sense a kind of minimality. We shall discuss on it in the subsequent paper.

(7.2) Let

$$
\tau(s)=\left(\begin{array}{rr}
2 & -1 \\
-1 & 2
\end{array}\right) \log s / 2 \pi \sqrt{-1}
$$

We consider $\mathscr{X}=\operatorname{Proj} R(\tau) / \Gamma, R(\tau)=\mathcal{O}\left[\xi_{m} \cdot \theta\right], \quad \xi_{m}=e\left(\frac{1}{2} m \tau\left(s^{2}\right)^{t} m+m^{t} \zeta\right)$. Then the theta series $\theta=\sum_{m} e\left(\frac{1}{2} m \tau\left(s^{2}\right)^{t} m+m^{t} \zeta\right)$ is a global section of $O(1)$ whose restriction on $\left(U_{0}\right)_{0}$ is given by, $\theta=\sum_{a \in \emptyset} x_{a}$, with the notations in proposition 2.3.

Let $\sigma_{i}=\left\langle P_{0}, P_{i}, P_{i+1}\right\rangle, a_{i}=\left\langle P_{0}, P_{i}\right\rangle, P_{i+6}=P_{i}$, where

$$
\begin{array}{lll}
P_{0}=(0,0), & P_{1}=(1,0), & P_{2}=(1,1), \quad P_{3}=(0,1), \\
P_{4}=(-1,0), & P_{5}=(-1,-1), & P_{6}=(0,-1) .
\end{array}
$$

Then the restriction of $\theta$ on $Z_{\sigma_{i}} \cap\left(U_{0}\right)_{0}$ is given by $1+x_{a_{i}}+x_{a_{i+1}}$. Hence a closed subscheme $C$ of $\mathscr{X}_{0}$ defined by $\theta=0$ is a union of two projective lines meeting transversally at three distinct points.

In the same way as above we can construct a family of stable curves of small genus. (see [15][17]).

We remark that a stable curve cannot necessarily be embedded into its corresponding stable quasi abelian variety.

\section{REFERENCES}

[1] M. Artin: Algebraization of formal moduli I, Global Analysis (Papers in honor of Kodaira) Univ. Tokyo Press, Princeton Univ. Press (1969), 21-71.

[2] - Algebraic approximation of structures over complete local rings volume Dédié au Professeur Oscas Zariski; Publ. math. I.H.E.S. No. 36, Paris, 1969, 23-58. l'université de Montréal (1973).

[ 3 ] — : Théorèmes de représentabilité pour les espaces algébriques, les presses de

[4] P. Deligne and D. Mumford: The irreducibility of the space of curves of given genus, Volume Dédié au Professeur Oscar Zariski, Publ. math. I.H.E.S., Paris, 1969, 75-110. 
[5] P. Deligne and M. Rapoport: Let schémas des modules de courbes elliptiques. Springer Berlin 349 (1972), 143-316.

[6] A. Grothendieck and J. Dieudonné: Elements de geometrie algebrique, Publ. Math. I.H.E.S., Paris 1960 ff.

[ 7 ] R. Hartshorne: Local cohomology, Springer Berlin.

[ 8 ] J. Igusa: A desingularization problem in the theory of Siegel modular functions, Math. Annalen 168 (1967), 228-260.

[ 9 ] —-: Theta functions, Springer Berlin, Band 194 (1972).

[10] - : Fiber systems of Jacobian varieties, Amer. J. Math. 81 (1959), 453-476.

[11] F. Knudsen, D. Mumford et al.: Troidal embeddings I, II, Springer Berlin.

[12] D. Mumford: Geometric invariant theory, Springer Berlin Band 34 (1970).

[13] —-: An analytic construction of degenerating abelian varieties over complete rings, Composito Math. Vol. 24, Fasc. 3 (1972), 239-272.

[14] _-: A new approach to compactifying locally symmetric varieties (to appear).

[15] I. Nakamura: On properfication of analytic Neron model and its application (in preparation).

[16] Y. Namikawa: On the canonical holomorphic map from the moduli space of stable curves to the Igusa monoidal transform, Nagoya Math. J., Vol. 52 (1973), 197-259.

[17] - : Studies on degeneration, Springer Berlin 412 (1974), 165-210.

[18] _-: A new compactification of the Siegel space and degeneration of abelian varieties (to appear).

[19] A. Neron: Model minimaux des varieties abeliennes sur les corps locaux et globaux, Publ. math. I.H.E.S. 21.

[20] T. Oda and C. S. Seshadri: Compactification of the generalized Jacobian variety (to appear).

[21] T. Oda and K. Miyake: Almost homogeneous algebraic varieties under algebraic torus action, Manifolds 1973, Proceedings of the International Conference on manifolds and related topics in topology, Univ. Tokyo Press, 1974.

[22] I. Satake: On the compactification of the Siegel space, J. Indian Math. Soc., Vol. 20 (1956), 259-281.

[23] M. Schlessinger: Functor of Artin rings, Trans. Amer. Math. Soc., Vol. 130 (1968), 208-222.

[24] H. W. Schuster: Deformationen analytischer Algebren, Invent. math. 6 (1968), 262-274.

[25] K. Ueno: On fiber spaces of normally polarized abelian varieties of dimension 2, I-II, J. Fac. Soc. Univ. Tokyo, Vols. 18 and 19 (1971-1972), 33-95 and 163-199.

[26] G. Voronoi: Nouvelles applicationss des paramètres continus à la théorie des formes quadratiques, I, II, III, J. Reine Angew. Math., Bd. 133 (1908), 97-108; Bd. 134 (1908), 198-287; Bd. 136 (1909), 67-181.

[27] J. Wavrik: A theorem of completeness for families of compact analytic spaces, Trans. Amer. Math. Soc. 163 (1972), 147-155.

[28] I. Satake: On the arithmetic of tube domains, Bulletin A.M.S., 79 (1973), 10761094.

[29] K. Ueno: Degenerated fibers of families of abelian varieties of dimension 2 (unpublished).

Mathematical Institute

Faculty of Science

Nagoya University 